\title{
A comparison of positivity in complex and tropical toric geometry
}

\author{
José Ignacio Burgos Gil ${ }^{1}$ (D) Walter Gubler $^{2}$ (D) Philipp Jell $^{2}$ (D) \\ Klaus Künnemann² ${ }^{2}$
}

Received: 27 March 2020 / Accepted: 26 January 2021 / Published online: 8 March 2021

(c) The Author(s) 2021

\begin{abstract}
Given a smooth complex toric variety we will compare real Lagerberg forms and currents on its tropicalization with invariant complex forms and currents on the toric variety. Our main result is a correspondence theorem which identifies the cone of invariant closed positive currents on the complex toric variety with closed positive currents on the tropicalization. In a subsequent paper, this correspondence will be used to develop a Bedford-Taylor theory of plurisubharmonic functions on the tropicalization.
\end{abstract}

Keywords Toric varieties · Tropicalization · Positive currents $\cdot$ Lagerberg forms

Mathematics Subject Classification $14 \mathrm{~L} 32 \cdot 14 \mathrm{~T} 05 \cdot 32 \mathrm{U} 05 \cdot 32 \mathrm{U} 40$

\section{Contents}

1 Introduction . . . . . . . . . . . . . . . . . . . . . . . . . . 1200

Notation and conventions . . . . . . . . . . . . . . . . . . . . . . . . . . . . . . . . . 1204

2 Positivity on real and complex vector spaces . . . . . . . . . . . . . . . . . . . . . . . 1205

J. I. Burgos was partially supported by MINECO research projects MTM2016-79400-P and by ICMAT Severo Ochoa project SEV-2015-0554. W. Gubler, P. Jell and K. Künnemann were supported by the collaborative research center SFB 1085 Higher Invariants - Interactions between Arithmetic Geometry and Global Analysis funded by the Deutsche Forschungsgemeinschaft.

$凶$ Klaus Künnemann

klaus.kuennemann@mathematik.uni-regensburg.de

José Ignacio Burgos Gil

burgos@icmat.es

Walter Gubler

walter.gubler@mathematik.uni-regensburg.de

Philipp Jell

philipp.jell@mathematik.uni-regensburg.de

1 Instituto de Ciencias Matemáticas (CSIC-UAM-UCM-UCM3), Calle Nicolás Cabrera 15, Campus de la Universidad Autónoma de Madrid, Cantoblanco, 28049 Madrid, Spain

2 Mathematik, Universität Regensburg, 93040 Regensburg, Germany 
3 Lagerberg forms and Lagerberg currents on partial compactifications . . . . . . . . . . . . . . 1214

4 Positivity for complex invariant forms and Lagerberg forms . . . . . . . . . . . . . . . . . . . 1221

5 Positivity for complex invariant currents and Lagerberg currents . . . . . . . . . . . . . . . . 1229

6 Tropicalization of positive currents . . . . . . . . . . . . . . . . . . . . . . . . 1237

7 The correspondence theorem for closed positive currents . . . . . . . . . . . . . . . . . . . . . 1246

Appendix A: Reminder about Radon and regular Borel measures . . . . . . . . . . . . . . . . . . 1253

References . . . . . . . . . . . . . . . . . . . . . . . 1254

\section{Introduction}

A smooth complex toric variety $X_{\Sigma}$ is a smooth algebraic variety over $\mathbb{C}$ with an open immersion of an algebraic torus $\mathbb{T}$ and an algebraic extension of the action of $\mathbb{T}$ on itself to $X_{\Sigma}$. Such a variety is encoded by the combinatorial structure of a fan $\Sigma$ in the vector space $N_{\mathbb{R}}:=N \otimes_{\mathbb{Z}} \mathbb{R}$ where $N$ is the cocharacter lattice of $\mathbb{T}$. We denote by $\mathbb{T}^{\text {an }}:=\mathbb{T}(\mathbb{C})$ and $X_{\Sigma}^{\text {an }}:=X_{\Sigma}(\mathbb{C})$ the respective complex analytic manifolds of complex points. Inside $\mathbb{T}^{\text {an }}$, there is a maximal compact torus $\mathbb{S}$. The quotient $X_{\Sigma}^{\text {an }} / \mathbb{S}$ is denoted by $N_{\Sigma}$. The topological space $N_{\Sigma}$ has a canonical stratification

$$
N_{\Sigma}=\coprod_{\sigma \in \Sigma} N(\sigma)
$$

indexed by the cones of $\Sigma$, analogous to the stratification of $X_{\Sigma}$ into orbits. The incidence relations between cones of $\Sigma$ translate to incidence relations between strata with the inclusions reversed. If $\tau$ is a face of the cone $\sigma \in \Sigma$, denoted as $\tau \prec \sigma$, then the corresponding strata satisfy $N(\sigma) \subset \overline{N(\tau)}$. The stratum corresponding to the cone $\{0\}$ is equal to $N_{\mathbb{R}}$. It is called the dense stratum. Each stratum $N(\sigma)$ of $N_{\Sigma}$ has a canonical structure of a finite dimensional real vector space equipped with a $\mathbb{Z}$-structure. For $\tau \prec \sigma \in \Sigma$, there is a linear projection

$$
\pi_{\sigma, \tau}: N(\tau) \longrightarrow N(\sigma) \text {. }
$$

The space $N_{\Sigma}$ is a classical object in the theory of toric varieties where it appears also under the name manifold with corners (see e.g. [1,17]]). In tropical geometry, it is called the Kajiwara-Payne tropicalization of $X_{\Sigma}$. By construction, it comes with a natural map trop: $X_{\Sigma}^{\text {an }} \rightarrow N_{\Sigma}$ which is a proper map of topological spaces.

On the spaces $X_{\Sigma}^{\text {an }}$ and $N_{\Sigma}$, there are sheaves of bigraded algebras of smooth differential forms. Both are denoted $A^{\cdot, \cdot}$ or, when we want to stress the underlying space, by $A_{X_{\Sigma}^{\prime}}^{\text {an }}$ and $A_{N_{\Sigma}}$ respectively. The well-known sheaf of complex smooth differential forms $A_{X_{\Sigma}^{\text {an }}}^{*}$ is a sheaf of $\mathbb{C}$-algebras and plays a central role in complex analysis and complex geometry. The sheaf $A_{N_{\Sigma}}^{*}$ is a sheaf of $\mathbb{R}$-algebras and was introduced by Smacka, Shaw and the third author [14], based on work of Lagerberg [16]. The smooth differential forms on $X_{\Sigma}^{\text {an }}$ are called complex forms while the forms on $N_{\Sigma}$ are called Lagerberg forms. In both cases, the elements of $A^{0,0}$ are called smooth functions.

We explain briefly the definition of Lagerberg forms. More details are given in Sect. 3 . If $U$ is an open subset of the finite dimensional real vector space $N(\sigma)$, Lagerberg [16] has introduced the bigraded $\mathbb{R}$-algebra

$$
A^{\cdot \cdot \cdot}(U):=\bigoplus_{p, q \in \mathbb{N}} A^{p}(U) \otimes_{C^{\infty}(U)} A^{q}(U)
$$


where $A^{\cdot}(U)$ denotes the usual $\mathbb{R}$-algebra of real smooth differential forms on $U$. For an open set $U \subset N_{\Sigma}$, denote by $U_{\sigma}:=N(\sigma) \cap U$ its strata. Then a Lagerberg form on $U$ is defined as a collection of forms $\left(\omega_{\sigma}\right)_{\sigma \in \Sigma}$, with $\omega_{\sigma} \in A^{\cdot} \cdot\left(U_{\sigma}\right)$, satisfying the following compatibility conditions. For every pair of cones $\tau \prec \sigma$ and every point $p \in U_{\sigma}$, there is a neighborhood $V \subset U$ of $p$ with $V_{\tau}=\pi_{\tau, \sigma}^{-1}\left(V_{\sigma}\right) \cap V$ and

$$
\omega_{\tau} \mid V_{\tau}=\pi_{\tau, \sigma}^{*}\left(\omega_{\sigma} \mid V_{\sigma}\right)
$$

on $V_{\tau}$. The compatibility conditions (1.1) are, roughly speaking, saying that close to the boundary, Lagerberg forms are constant in the direction towards the boundary. Although this condition does not seem entirely natural from an archimedean point of view, it is very natural from both a tropical [14] and a non-archimedean point of view [13]. Moreover, it has very strong consequences. For instance, if $\omega$ is a form of bidegree $(p, q)$, then the support of $\omega$ is disjoint to any stratum of dimension smaller than $\min (p, q)$.

There are natural differential operators $d^{\prime}, d^{\prime \prime}$ of bidegree $(1,0)$ and $(0,1)$ turning $A_{N_{\Sigma}}$ into a double complex analogous to the usual differential operators $\partial$ and $\bar{\partial}$ on $A_{X_{\Sigma}}$. There is also a theory of integration for Lagerberg forms similarly to the complex case.

The sheaf $A_{X_{\Sigma}^{\prime a n}}^{*}$ has an antilinear involution, the complex conjugation, that sends $A^{p, q}$ to $A^{q, p}$. A complex form is real, if it is invariant under complex conjugation. The sheaf $A_{N_{\Sigma}}$, has also a canonical involution $J$ called the Lagerberg involution. A form $\omega$ of bidegree $(p, p)$ with $J(\omega)=(-1)^{p} \omega$ is called symmetric. In both settings, there is a notion of positivity for $(p, p)$-forms (see 4.2.6). Positive forms on $X_{\Sigma}^{\text {an }}$ are always real, while positive forms on $N_{\Sigma}$ are always symmetric.

In Sect. 2.3 and in Sect. 4.2, we will introduce a new antilinear involution $F$ on the sheaf of $\mathbb{S}$-invariant forms on $X_{\Sigma}^{\text {an }}$ that respects the bigrading, and anticommutes with complex conjugation on one-forms. We denote by $A_{X_{\Sigma}^{\text {an }}}^{\mathbb{S}, F}$ the subsheaf of $\mathbb{S}$-invariant forms which are $F$-invariant.

Theorem A Let $U \subset N_{\Sigma}$ be an open subset and $V$ the $\mathbb{S}$-invariant open subset $V:=$ $\operatorname{trop}^{-1}(U)$ of $X_{\Sigma}^{\mathrm{an}}$. There exists a unique bigraded algebra morphism

$$
\text { trop*: } A^{\cdot, \cdot}(U) \longrightarrow A^{\cdot, \cdot}(V)^{\mathbb{S}, F}
$$

with trop* $\varphi=\varphi \circ$ trop for all $\varphi \in A^{0,0}(U)$ and which satisfies

$$
\text { trop* } \circ d^{\prime}=\pi^{-1 / 2} \partial \circ \text { trop*}^{*}, \text { trop* }^{*} \circ d^{\prime \prime}=\pi^{-1 / 2} i \bar{\partial} \circ \text { trop*. }
$$

Moreover, for $\omega \in A^{p, q}(U)$, we have

$$
\operatorname{trop}^{*}(J(\omega))=i^{p+q} \overline{\operatorname{trop}^{*}(\omega)} .
$$

Therefore trop* sends symmetric forms to real forms. Furthermore, this morphism respects positivity and integration of top dimensional forms.

If $U$ is contained in the dense stratum $N_{\mathbb{R}}$, then (1.2) is an isomorphism. In general, this is no longer true. The reason for this is the compatibility conditions (1.1). These results will be shown in Section 4. The normalization factors in equation (1.3) are almost forced by the compatibility with integration and the compatibility between the Lagerberg involution and complex conjugation (1.4). If we do not insist on compatibility with integration or with the bigrading, other identifications between Lagerberg forms and invariant complex forms are possible. For instance, the map (1.2) differs from the interpretation of Lagerberg forms as $\mathbb{S}$-invariant forms given in [7, Remarque (1.2.12)]. 
The main interest of this paper will be currents. To define currents on an open subset $U \subset N_{\Sigma}$, we first introduce a topology on the space of Lagerberg forms with compact support $A_{c}^{\cdot{ }^{\prime}}(U)$. The definition is similar to the complex case with additional input caused by the compatibility condition (1.1) towards the boundary (see Subsection 3.2 for details). A Lagerberg current of type $(p, q)$ on $U$ is a continuous linear map $T: A_{c}^{n-p, n-q}(U) \rightarrow \mathbb{R}$. We denote the space of currents of type $(p, q)$ by $D^{p, q}(U)$. By duality, the involution $J$ defines an involution on $D^{\prime,}$, hence a notion of symmetric currents. We call $T \in D^{p, p}(U)$ positive, if it is symmetric and $T(\alpha) \geq 0$ for all positive Lagerberg forms $\alpha \in A_{c}^{n-p, n-p}(U)$.

Let again $V=\operatorname{trop}^{-1}(U)$. Since the map trop is proper, the dual of the map trop* from (1.2) induces a $\mathbb{C}$-linear map

$$
\operatorname{trop}_{*}: D^{p, q}(V) \longrightarrow D^{p, q}(U) \otimes_{\mathbb{R}} \mathbb{C}
$$

defined by $\operatorname{trop}_{*}(T)(\alpha)=T\left(\operatorname{trop}^{*}(\alpha)\right)$ for all $T \in D^{p, q}(V)$ and $\alpha \in A_{c}^{n-p, n-q}(V)$. We will show that $\operatorname{trop}_{*}(T) \in D^{p, p}(U)$ for every $\mathbb{S}$-invariant $T \in D^{p, p}(V)$ which is also $F$-invariant. Since the map trop* $: A_{c}^{p, q}(U) \rightarrow A_{c}^{p, q}(V)$, albeit injective, is not a closed immersion, the map trop ${ }_{*}$ is in general not surjective. Since trop* preserves positivity of

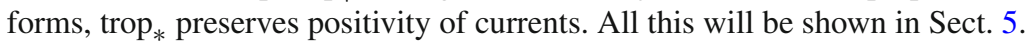

The main result of this paper is the following Correspondence Theorem.

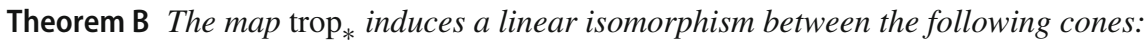

(i) The cone of $\mathbb{S}$-invariant positive complex currents in $D^{p, p}(V)$ that are closed with respect to $\partial$ and $\bar{\partial}$ and that are invariant with respect to $F$.

(ii) The cone of positive Lagerberg currents in $D^{p, p}(U)$ that are closed with respect to $d^{\prime}$ and $d^{\prime \prime}$.

The proof of Theorem B will be given in Theorem 7.1.5. We will show in Examples 5.1.11 and 5.1.12 that we cannot omit any of the conditions closed or positive in Theorem B.

Let us give some details about the proof of the Correspondence Theorem. In the case $p=n:=\operatorname{dim} X_{\Sigma}$, the cone in (i) is the space of positive $\mathbb{S}$-invariant Radon measures on $V$ and the cone in (ii) is the space of positive Radon measures on $U$, hence Theorem B follows readily from the fact that $U$ is the quotient of $V$ by the $\mathbb{S}$-action (see Corollary 5.1.17). This suggests that for $p<n$, we consider coefficients of complex and Lagerberg currents to follow a similar argument. In fact, it is more convenient to go for the dual notion of co-coefficients as follows:

For simplicity, we consider the case $X_{\Sigma}^{\text {an }}=\mathbb{C}^{n}$ with coordinates $z=\left(z_{1}, \ldots, z_{n}\right)$, a situation that can always be achieved locally. Then $N_{\Sigma}=(\mathbb{R} \cup\{\infty\})^{n}$ has coordinates $\left(u_{1}, \ldots, u_{n}\right)$ and the tropicalization map is given by

$$
\operatorname{trop}(z)=\left(-\log \left|z_{1}\right|, \ldots,-\log \left|z_{n}\right|\right) .
$$

The cones of $\Sigma$ are the faces of $\mathbb{R}_{\geq 0}^{n}$. Any cone in $\Sigma$ has the form $\sigma_{L}:=\left\{u \in \mathbb{R}_{\geq 0}^{n} \mid u_{i}=\right.$ $0 \forall i \notin L\}$ for some $L \subset\{1, \ldots, n\}$ and the corresponding stratum of $N_{\Sigma}$ is given by

$$
N\left(\sigma_{L}\right):=\left\{\left(u_{1}, \ldots, u_{n}\right) \in N_{\Sigma} \mid u_{i}=\infty \text { if and only if } i \in L\right\} .
$$

For $M \subset\{1, \ldots, n\}$, we define the following union of strata of codimension 1:

$$
E^{M}:=\left\{\left(u_{1}, \ldots, u_{n}\right) \in N_{\Sigma} \mid u_{i}=\infty \text { for some } i \in M\right\} .
$$

Let $U$ be an open subset of $(\mathbb{R} \cup\{\infty\})^{n}$. Given a Lagerberg current $T \in D^{p, p}(U)$ and $I, J \subset\{1, \ldots, n\}$ with $|I|=|J|=n-p$, we call $T^{I J} \in D^{n, n}\left(U \backslash E^{I \cup J}\right)$ given by

$$
T^{I J}(f)=T\left((-1)^{q(q-1) / 2} f d^{\prime} u_{I} \wedge d^{\prime \prime} u_{J}\right)
$$


a co-coefficient of $T$. If the Lagerberg current $T$ is positive, then the co-coefficients $T^{I J}$ are real Radon measures, $T^{I J}=T^{J I}$, the $T^{I I}$ are positive Radon measures and $2\left|T^{I J}\right| \leq$ $T^{I I}+T^{J J}$, where $\left|T^{I J}\right|$ denotes the total variation measure of $T^{I J}$ (see Subsection 5.2).

A key ingredient in the proof of the Correspondence Theorem is the following Decomposition Theorem along the above stratification.

Theorem C Let $U$ be an open subset of $(\mathbb{R} \cup\{\infty\})^{n}$. If $T$ is a positive current in $D^{p, p}(U)$, then there is a decomposition

$$
T=\sum_{\sigma \in \Sigma} T_{\sigma}
$$

with uniquely determined currents $T_{\sigma}$ such that $U \backslash\left(E^{I \cup J} \cup N(\sigma)\right)$ is a null set with respect to the Radon measure $T_{\sigma}^{I J}$ for any $\sigma=\sigma_{L} \in \Sigma$.

The decomposition (1.6) does not depend on the choice of the coordinates $u_{1}, \ldots, u_{n}$ hence gives a canonical decomposition for any positive current $T \in D^{p, p}(U)$ on any open subset $U$ of $N_{\Sigma}$ as we show in Theorem 6.1.6. A similar statement is well known on the complex toric manifold $X_{\Sigma}^{\text {an }}$ and we show in Sect. 6.1 that both canonical decompositions are closely related via trop .

For a positive Lagerberg current $T \in D^{p, p}(U)$, we will prove that $T=\operatorname{trop}_{*}(S)$ for a positive current $S$ on $V=\operatorname{trop}^{-1}(U)$ if and only if $T$ has $\mathbb{C}$-finite local mass. The latter is a local condition on $U$ given in Definition 6.2.1. We then show that a closed positive Lagerberg current has $\mathbb{C}$-finite local mass, completing the proof of surjectivity. For injectivity of trop , an additional argument is required. All this is done in Sect. 7.1.

As an application, we will prove in Theorem 7.2.4 a tropical analogue of the Skoda-El Mir Theorem for Lagerberg currents in the toric setting: Let $U$ be an open subset of $N_{\Sigma}$ and let $E$ be a union of strata closures in $N_{\Sigma}$. We consider a closed positive current $T \in D^{p, p}(U \backslash E)$ which has $\mathbb{C}$-finite local mass on $U$. Then we can extend $T$ by zero to a closed positive Lagerberg current on $U$. For details, we refer to Sect. 7.2. A consequence of the Tropical Skoda-El Mir theorem is that in the canonical decomposition (1.6), if $T$ is closed, then all the currents $T_{\sigma}$ are closed.

The motivation for the present work is the following. It is known that there is no way to continuously extend the wedge product on $A^{\cdot, \cdot}$ to $D^{\cdot, \cdot}$. Bedford-Taylor theory provides a way to define products of certain closed positive currents on $X_{\Sigma}^{\text {an }}$. In a subsequent paper, using the Correspondence Theorem and Bedford-Taylor theory for complex manifolds, we develop a Bedford-Taylor theory on $N_{\Sigma}$.

For instance, Bedford-Taylor theory on $N_{\Sigma}$ will have the following application: Let $K$ be a field endowed with a non-archimedean complete absolute value ||$_{v}$. From the fan $\Sigma$, we may construct a toric variety $X_{\Sigma, K}$. We may also consider the analytification $X_{\Sigma, K}^{\text {an }}$ of the toric variety $X_{\Sigma, K}$ as a Berkovich space and the corresponding tropicalization map

$$
\operatorname{trop}_{K}: X_{\Sigma, K}^{\mathrm{an}} \longrightarrow N_{\Sigma}
$$

which should be viewed as a non-archimedean analogue of the tropicalization map trop of the complex toric manifold $X_{\Sigma}^{\text {an }}$ considered before. For any open subset $U$ of $N_{\Sigma}$, these tropicalization maps lead to a natural bijective correspondence between invariant continuous plurisubharmonic functions on $\operatorname{trop}^{-1}(U)$ and invariant continuous plurisubharmonic functions on $\operatorname{trop}_{K}^{-1}(U)$ in the sense of Chambert-Loir and Ducros [7, §5.5]. Moreover, we show that the complex Bedford-Taylor theory corresponds to the Bedford-Taylor theory of Chambert-Loir and Ducros [7, §5.6]. 
We explain in more detail the content of the paper. In Sect. 2, we introduce complex and Lagerberg multilinear forms. We interpretate the Lagerberg forms as the complex forms which are invariant under a natural involution $F$. Then we discuss several positivity notions of these forms. In the complex case, weakly positive, positive and strongly positive multilinear forms are well-known. They form strictly convex cones of maximal dimension. Moreover, the cone of strongly positive forms is dual to the one of weakly positive forms, while the cone of positive forms is self dual. Similar notions are defined in [16] for Lagerberg multilinear forms on a real vector space. The notions of weakly and strongly positive Lagerberg forms are however somewhat pathological. We show in Example 2.3.6 that the cone of strongly positive forms is not of full dimension and that the cone of weakly positive forms is not strictly convex. This also implies that the notions of weakly and strongly positive in the complex and Lagerberg case do not correspond exactly. For this reason, we will mainly restrict ourselves to positive forms.

In Sect. 3, we will first introduce the partial compactification $N_{\Sigma}$ associated to a fan $\Sigma$ and we will describe its topology. Then we introduce Lagerberg forms on $N_{\Sigma}$. Similarly as in complex analysis, we endow the space of compactly supported Lagerberg forms with a locally convex topology and we define the dual notion of Lagerberg currents. The upshot of this section is that Lagerberg forms and currents on $N_{\Sigma}$ satisfy similar properties as their complex analogues.

In Sect. 4, we study positivity of Lagerberg forms and compare them to invariant complex differential forms. In particular, we prove Theorem A. We first deal with the dense torus $\mathbb{T}$ in Sect. 4.1 before we consider arbitrary smooth toric varieties in Sect. 4.2.

Section 5 is devoted to positivity of Lagerberg currents and the relation with positivity of complex currents. We define the map trop ${ }_{*}$ and discuss the compatibility of the different notions of positivity with respect to this map. We also define the co-coefficients of a Lagerberg current and show that, analogously to the complex case, the co-coefficients of a positive Lagerberg current are Radon measures. We show in Example 5.2.8 that there are weakly positive Lagerberg currents whose co-coefficients are not Radon measures. This is caused by the fact that the cone of weakly positive multilinear Lagerberg forms is not strictly convex.

In Sect. 6, we prove the decomposition theorem (see Theorem C). Moreover we introduce the concept of $\mathbb{C}$-finite local mass. Intuitively, a current has $\mathbb{C}$-finite local mass if it has local finite mass as an invariant complex current (see Definition 6.2.1 for details). We give in Example 6.2.3 a positive current $T$ that does not have $\mathbb{C}$-finite local mass. Nevertheless, it is of the form $T=\operatorname{trop}_{*}(S)$ for a complex current $S$, but this current cannot be chosen to be positive.

Finally, Sect. 7 is devoted to the proof of Theorem B and of the tropical Skoda-El Mir theorem. In Appendix A, there is a reminder on Borel and Radon measures.

\section{Notation and conventions}

The set $\mathbb{N}$ of natural numbers includes zero. We write $\mathbb{R}_{\infty}=\mathbb{R} \cup\{\infty\}$ and $\mathbb{R}_{\geq t}:=\{u \in \mathbb{R} \mid$ $u \geq t\}$ for any $t \in \mathbb{R}$. In the notation $A \subset B$, we allow that $A=B$.

In this paper, $N$ usually denotes a free abelian group of rank $n$ with dual $M$. We denote by $N_{\mathbb{R}}$ and $M_{\mathbb{R}}$ their scalar extensions to $\mathbb{R}$. By a fan $\Sigma$ in $N_{\mathbb{R}}$, we mean a fan consisting of strictly convex rational polyhedral cones in $N_{\mathbb{R}}$. We denote the associated (complex) toric variety by $X_{\Sigma}$ and the associated partial compactification of $N_{\mathbb{R}}$ by $N_{\Sigma}$ (see Sect. 3.1). 
A topological space is called locally compact if it has a basis consisting of relatively compact subsets; but it is not necessarily Hausdorff. A topological vector space is assumed to be Hausdorff. Our conventions on Radon measures are summarized in Appendix A.

\section{Positivity on real and complex vector spaces}

Let $V$ be a real vector space of dimension $n$. Write $V_{\mathbb{C}}=V \otimes_{\mathbb{R}} \mathbb{C}$ for the associated complex vector space, $V^{*}=\operatorname{Hom}_{\mathbb{R}}(V, \mathbb{C})=\operatorname{Hom}_{\mathbb{C}}\left(V_{\mathbb{C}}, \mathbb{C}\right)$ for the complex dual and $V^{\prime}=\operatorname{Hom}_{\mathbb{R}}(V, \mathbb{R})$ for the real dual. Let $\overline{V_{\mathbb{C}}}$ denote the real vector space underlying $V_{\mathbb{C}}$ with the complex structure determined by $\lambda(v \otimes \mu)=v \otimes(\bar{\lambda} \mu)$. We denote the complex dual of $\overline{V_{\mathbb{C}}}$ by $\bar{V}^{*}$. Observe that $\bar{V}^{*}$ agrees with the space of antilinear maps from $V_{\mathbb{C}}$ to $\mathbb{C}$. Let $V^{\prime \prime}$ be a copy of $V^{\prime}$. We consider the exterior algebras

$$
\Lambda^{, \cdot} V^{*}:=\Lambda_{\mathbb{C}}\left(V^{*} \oplus \bar{V}^{*}\right)=\bigoplus_{p, q \in \mathbb{N}} \Lambda^{p, q} V^{*},
$$

and

$$
\Lambda^{\cdot, \cdot} V^{\prime}:=\Lambda_{\mathbb{R}}\left(V^{\prime} \oplus V^{\prime \prime}\right)=\bigoplus_{p, q \in \mathbb{N}} \Lambda^{p, q} V^{\prime} .
$$

The elements of $\Lambda^{p, q} V^{*}$ are called complex $(p, q)$-forms while the elements of $\Lambda^{p, q} V^{\prime}$ are called Lagerberg $(p, q)$-forms.

\subsection{The complex situation}

We recall some definitions from complex geometry.

The identity induces antilinear maps $\sigma: V_{\mathbb{C}} \rightarrow \overline{V_{\mathbb{C}}}$ and $\sigma: \Lambda^{p} V_{\mathbb{C}} \rightarrow \Lambda^{p} \overline{V_{\mathbb{C}}}$ which we denote by $w \mapsto \bar{w}$. The inverse of $\sigma$ is also denoted by $\sigma: \overline{V_{\mathbb{C}}} \rightarrow V_{\mathbb{C}}$. The antilinear maps $\sigma$ induce antilinear maps $\sigma: V^{*} \rightarrow \bar{V}^{*}$ and $\sigma: \bar{V}^{*} \rightarrow V^{*}$, that extend uniquely to an antilinear involution of the $\mathbb{R}$-algebra $\Lambda^{*, \cdot} V^{*}$. This involution sends $\Lambda^{p, q} V^{*}$ to $\Lambda^{q, p} V^{*}$. We continue to write $\sigma(\omega)=\bar{\omega}$ for complex forms $\omega$. A complex form $\omega$ is called real if $\bar{\omega}=\omega$.

Choose a real basis $e_{1}, \ldots, e_{n}$ of $V$. We obtain dual complex bases $d u_{1}, \ldots, d u_{n}$ of $V^{*}$ and $d \bar{u}_{1}, \ldots, d \bar{u}_{n}$ of $\bar{V}^{*}$ determined by $d u_{i}\left(e_{j} \otimes 1\right)=\delta_{i j}=d \bar{u}_{i}\left(e_{j} \otimes 1\right)$. Then we have

$$
\sigma\left(\lambda d u_{i}\right)=\bar{\lambda} d \bar{u}_{i} .
$$

Definition 2.1.1 The canonical orientation of the vector space $V_{\mathbb{C}}$ is the orientation determined by the real form

$$
\omega_{n}=d u_{1} \wedge i d \bar{u}_{1} \wedge \cdots \wedge d u_{n} \wedge i d \bar{u}_{n} \in \Lambda^{n, n} V^{*} .
$$

The form $\omega_{n}$ depends on our choice of a basis, but the orientation does not.

An $\omega \in \Lambda^{p, p} V^{*}$ is called strongly positive if it is in the convex cone spanned by

$$
\left\{\alpha_{1} \wedge i \bar{\alpha}_{1} \wedge \cdots \wedge \alpha_{p} \wedge i \bar{\alpha}_{p} \mid \alpha_{j} \in \Lambda^{1,0} V^{*} \text { for } j=1, \ldots, p\right\} .
$$

An $\omega \in \Lambda^{p \cdot p} V^{*}$ is called positive if it belongs to the convex cone spanned by

$$
\left\{i^{p^{2}} \alpha \wedge \bar{\alpha} \mid \alpha \in \Lambda^{p, 0} V^{*}\right\} .
$$


A complex $(p, p)$-form $\omega \in \Lambda^{p, p} V^{*}$ is called weakly positive if, for every strongly positive form $\eta$ of type $(n-p, n-p)$, there is a real number $\gamma \geq 0$ with

$$
\omega \wedge \eta=\gamma \omega_{n} .
$$

We denote by $\Lambda_{+, s}^{p, p} V^{*}, \Lambda_{+}^{p, p} V^{*}$ and $\Lambda_{+, w}^{p, p} V^{*}$ the cones of strongly positive, positive and weakly positive $(p, p)$-forms respectively.

Observe that our weakly positive forms are called positive in [9, §III.1.A].

Definition 2.1.2 To $\eta \in \Lambda^{p, p} V^{*}$ we associate a sesquilinear form $|\eta|$ on $\Lambda^{p} V_{\mathbb{C}}$ by the rule

$$
|\eta|(x, y)=(-1)^{\frac{p(p-1)}{2}} i^{-p} \eta(x, \bar{y}) .
$$

Moreover, for $q=n-p$, there is a duality pairing

$$
\langle\cdot, \cdot\rangle: \Lambda^{p, p} V^{*} \otimes \Lambda^{q, q} V^{*} \longrightarrow \mathbb{C}
$$

defined by

$$
\eta \wedge \omega=\langle\eta, \omega\rangle \omega_{n} .
$$

We denote by $\Lambda^{p, p} V_{\mathbb{R}}^{*}$ the subspace of real elements. Then the pairing (2.1) induces a pairing

$$
\langle\cdot, \cdot\rangle_{\mathbb{R}}: \Lambda^{p, p} V_{\mathbb{R}}^{*} \otimes \Lambda^{q, q} V_{\mathbb{R}}^{*} \rightarrow \mathbb{R} .
$$

Note that the assignment $\eta \mapsto|\eta|$ is canonical and gives an isomorphism between $\Lambda^{p, p} V^{*}$ and the space of sesquilinear forms on $\Lambda^{p} V_{\mathbb{C}}$. On the other hand, the duality pairing $\langle\cdot, \cdot\rangle$ depends on the choice of basis but only up to a non-zero positive number.

Proposition 2.1.3 A form $\eta \in \Lambda^{p, p} V^{*}$ is real if and only if the sesquilinear form $|\eta|$ is Hermitian. A form $\eta$ is positive if and only if $|\eta|$ is a positive semidefinite Hermitian form.

Proof The antilinear involution $\sigma$ on $\Lambda^{p, p} V^{*}$ is given by

$$
\sigma(\eta)(x, \xi)=\bar{\eta}(x, \xi)=(-1)^{p} \overline{\eta(\bar{\xi}, \bar{x})}
$$

for $x \in \Lambda^{p} V_{\mathbb{C}}$ and $\xi \in \Lambda^{p} \overline{V_{\mathbb{C}}}$. Assume that $\eta$ is real. Then for $x, y \in \Lambda^{p} V_{\mathbb{C}}$ we have

$$
\begin{aligned}
\overline{|\eta|(x, y)} & =\overline{(-1)^{\frac{p(p-1)}{2}} i-p \eta(x, \bar{y})}=(-1)^{\frac{p(p-1)}{2}}(-i)^{-p} \overline{\eta(x, \bar{y})} \\
& =(-1)^{\frac{p(p-1)}{2}}(-i)^{-p}(-1)^{p} \bar{\eta}(y, \bar{x})=(-1)^{\frac{p(p-1)}{2}} i^{-p} \eta(y, \bar{x})=|\eta|(y, x) .
\end{aligned}
$$

Thus, the sesquilinear form $|\eta|$ is hermitian. The converse is proved analogously.

Assume now that $\eta \in \Lambda^{p, p} V^{*}$ is a positive form and $x \in \Lambda^{p} V_{\mathbb{C}}$. Then we can write

$$
\eta=\sum_{j} i^{p^{2}} \gamma_{j} \alpha_{j} \wedge \bar{\alpha}_{j}
$$

with $\gamma_{j} \in \mathbb{R}_{\geq 0}$ and $\alpha_{j} \in \Lambda^{p, 0} V^{*}$. Then, since $i^{p^{2}}(-1)^{\frac{p(p-1)}{2}} i^{-p}=1$, we have

$$
|\eta|(x, x)=(-1)^{\frac{p(p-1)}{2}} i^{-p} \eta(x, \bar{x})=\sum_{j} \gamma_{j}\left(\alpha_{j} \wedge \bar{\alpha}_{j}\right)(x, \bar{x})=\sum_{j} \gamma_{j} \alpha_{j}(x) \overline{\alpha_{j}(x)} \geq 0,
$$

proving that $|\eta|$ is positive semidefinite. 
Conversely, assume that $|\eta|$ is positive semidefinite. Then by the spectral theory of Hermitian forms, there are $\gamma_{j} \in \mathbb{R}_{\geq 0}$ and $\alpha_{j} \in \Lambda^{p, 0} V^{*}=\left(\Lambda^{p} V_{\mathbb{C}}\right)^{*}$ such that

$$
|\eta|=\sum \gamma_{j} \alpha_{j} \otimes \bar{\alpha}_{j}
$$

This implies that

$$
\eta=\sum i^{p^{2}} \gamma_{j} \alpha_{j} \wedge \bar{\alpha}_{j}
$$

showing that $\eta$ is a positive form.

Lemma 2.1.4 For every $p \geq 0$, the complex vector space $\Lambda^{p, p} V^{*}$ admits a $\mathbb{C}$-basis of strongly positive forms.

Proof [9, Lemma III.1.4].

Lemma 2.1.5 Any weakly positive form in $\Lambda^{p, p} V^{*}$ is real.

Proof The fact that $\omega_{n}$ and any positive or strongly positive form are real follows directly from the definition. Let now $\omega$ be a weakly positive form in $\Lambda^{p, p} V^{*}$. This means that for each strongly positive form $\eta$ in $\Lambda^{q, q} V^{*}$, there is a non-negative real number $\gamma$ such that

$$
\omega \wedge \eta=\gamma \omega_{n} .
$$

Since $\eta, \gamma$ and $\omega_{n}$ are real, this implies that

$$
\bar{\omega} \wedge \eta=\gamma \omega_{n} .
$$

Hence $(\omega-\bar{\omega}) \wedge \eta=0$ for any strongly positive form $\eta$ in $\Lambda^{q, q} V^{*}$. By Lemma 2.1.4, $\Lambda^{q, q} V^{*}$ admits a basis of strongly positive elements. Therefore $(\omega-\bar{\omega}) \wedge \eta=0$ holds for any $\eta \in \Lambda^{q, q} V^{*}$. By duality, we get $\omega-\bar{\omega}=0$ and hence $\omega$ is real.

Corollary 2.1.6 For $p \in \mathbb{N}$, there are inclusions of closed convex cones

$$
\Lambda_{+, s}^{p, p} V^{*} \subset \Lambda_{+}^{p, p} V^{*} \subset \Lambda_{+, w}^{p, p} V^{*}
$$

in $\Lambda^{p, p} V_{\mathbb{R}}^{*}$. For $q:=n-p$, the cones $\Lambda_{+, s}^{p, p} V^{*}$ and $\Lambda_{+, w}^{q, q} V^{*}$ are dual to each other and the cone $\Lambda_{+}^{p, p} V^{*}$ is the dual of $\Lambda_{+}^{q, q} V^{*}$ with respect to the real duality pairing (2.2).

Proof By definition, the spaces of strongly positive forms and of positive forms are convex cones contained in $\Lambda^{p, p} V_{\mathbb{R}}^{*}$. Lemma 2.1.5 implies that $\Lambda_{+, w}^{p, p} V^{*}$ is contained in $\Lambda^{p, p} V_{\mathbb{R}}^{*}$. Then $\Lambda_{+, w}^{p, p} V^{*}$ is a closed convex cone as the dual of the convex cone $\Lambda_{+, w}^{p, p} V^{*}$.

We next show that the convex cone of strongly positive forms is closed. Choose any hermitian metric in the complex vector space $\Lambda^{p} V^{*}$ and let $S \subset \Lambda^{p} V^{*}$ be the unit sphere. The set $K \subset S$ of totally decomposable elements is closed as it is the preimage of the Grassmanian $\operatorname{Gr}\left(p, V^{*}\right)$ under the projection $S \rightarrow \mathbb{P}\left(\Lambda^{p} V^{*}\right)$. Since $S$ is compact, so is $K$. Let $K^{\prime} \subset \Lambda^{p, p} V^{*}$ be the image of $K$ under the continuous map

$$
\Lambda^{p} V^{*} \rightarrow \Lambda^{p, p} V^{*}, \quad \alpha \mapsto i^{p^{2}} \alpha \wedge \bar{\alpha} .
$$

Then $K^{\prime}$ is a compact set that does not contain 0 . Thus the convex cone over $K^{\prime}$ is closed [19, Cor. 9.6.1]. Since the convex cone over $K^{\prime}$ is $\Lambda_{+, s}^{p, p} V^{*}$, we deduce that the space of strongly positive forms is a closed convex cone. Being $\Lambda_{+, s}^{p, p} V^{*}$ closed and convex, it agrees with its double dual. Therefore $\Lambda_{+, s}^{p, p} V^{*}$ is the dual of $\Lambda_{+, w}^{p, p} V^{*}$. 
We next prove that $\Lambda_{+}^{p, p} V^{*}$ is self dual. Let $\gamma \in \Lambda^{n} V^{*}$. Then $\gamma=z d u_{1} \wedge \cdots \wedge d u_{n}$ for some $z \in \mathbb{C}$ and hence $i^{n^{2}} \gamma \wedge \bar{\gamma}=z \bar{z} \omega_{n}$. For $\alpha \in \Lambda^{p} V^{*}$ and $\beta \in \Lambda^{q} V^{*}$, we conclude that

$$
i^{p^{2}} i^{q^{2}} \alpha \wedge \bar{\alpha} \wedge \beta \wedge \bar{\beta}=i^{n^{2}} \alpha \wedge \beta \wedge \overline{\alpha \wedge \beta}
$$

is a positive multiple of $\omega_{n}$. Here we have used the identity $i^{p^{2}} i^{q^{2}}(-1)^{p q}=i^{n^{2}}$. This proves that the cone $\Lambda_{+}^{p, p} V^{*}$ is contained the dual of $\Lambda_{+}^{q, q} V^{*}$. To prove the converse, we introduce the isomorphism $\varphi: \Lambda^{q} V^{*} \rightarrow \Lambda^{p} V$ defined, for $\alpha \in \Lambda^{q} V^{*}$, by

$$
\beta \wedge \alpha=\beta(\varphi(\alpha)) d u_{1} \wedge \cdots \wedge d u_{n} \quad\left(\beta \in \Lambda^{p} V^{*}\right) .
$$

Then, for any pair of forms $\eta \in \Lambda^{p, p} V^{*}$ and $\alpha \in \Lambda^{q} V^{*}$, the equality

$$
\eta \wedge i^{q^{2}} \alpha \wedge \bar{\alpha}=|\eta|(\varphi(\alpha), \varphi(\alpha)) \omega_{n}
$$

is satisfied. Therefore, if $\eta$ belongs to the dual cone of $\Lambda_{+}^{q, q} V^{*}$, then the sesquilinear form $|\eta|$ is positive semidefinite. By Proposition 2.1.3, the form $\eta$ is positive proving the reverse inclusion. We deduce that the cone $\Lambda_{+}^{p, p} V^{*}$ is closed because it is a dual cone.

Remark 2.1.7 For $p=0,1, n-1, n$, the notions of strong positivity, positivity and weakly positivity agree (see [9, Corollary III.1.9]). In [9, Remark III.1.10], there are examples of positive forms that are not strongly positive for any $2 \leq p \leq n-2$.

\subsection{The real situation}

We now shift to positivity of Lagerberg forms following [16]. We consider again a real basis $e_{1}, \ldots, e_{n}$ of $V$ which induces dual bases $d^{\prime} u_{1}, \ldots, d^{\prime} u_{n}$ of $V^{\prime}$ and $d^{\prime \prime} u_{1}, \ldots, d^{\prime \prime} u_{n}$ of $V^{\prime \prime}$.

We denote by $J V$ another copy of our $n$-dimensional $\mathbb{R}$-vector space $V$ and let us denote by $J: V \mapsto J V$ the identity map. There is a unique involution on $V \oplus J V$ that extends $J$ and which we also denote by $J$. From now on, we make the identification $V^{\prime \prime}:=\operatorname{Hom}(J V, \mathbb{R})$ and then duality yields an involution on $V^{\prime} \oplus V^{\prime \prime}$ which we also call $J$. There is a unique algebra homomorphism on $\Lambda^{\cdot, \cdot} V^{\prime}$ that extends $J$. It is again an involution mapping $\Lambda^{p, q} V^{\prime}$ onto $\Lambda^{q, p} V^{\prime}$. This map, also denoted by $J$, is called the Lagerberg involution.

Definition 2.2.1 The Lagerberg orientation is the orientation on the vector space $V^{\prime} \oplus V^{\prime \prime}$ defined by

$$
\tau_{n}=d^{\prime} u_{1} \wedge d^{\prime \prime} u_{1} \wedge \cdots \wedge d^{\prime} u_{n} \wedge d^{\prime \prime} u_{n} \in \Lambda^{n, n}(V) .
$$

Consider a form $\omega \in \Lambda^{p, p} V^{\prime}$. We call $\omega$ symmetric if

$$
J(\omega)=(-1)^{p} \omega .
$$

We call $\omega$ strongly positive if it belongs to the convex cone spanned by

$$
\left\{\alpha_{1} \wedge J\left(\alpha_{1}\right) \wedge \cdots \wedge \alpha_{p} \wedge J\left(\alpha_{p}\right) \mid \alpha_{j} \in \Lambda^{1,0} V^{\prime} \text { for } j=1, \ldots, p\right\} .
$$

We call $\omega$ positive if it belongs to the convex cone spanned by

$$
\left\{(-1)^{\frac{p(p-1)}{2}} \alpha \wedge J(\alpha) \mid \alpha \in \Lambda^{p, 0} V^{\prime}\right\} .
$$

A symmetric Lagerberg $(p, p)$-form $\omega \in \Lambda^{p, p} V^{\prime}$ is called weakly positive if for every strongly positive form $\eta$ of type $(n-p, n-p)$, there is a real number $\gamma \geq 0$ with

$$
\omega \wedge \eta=\gamma \tau_{n} .
$$


We will also denote as $\Lambda_{+, s}^{p, p} V^{\prime}, \Lambda_{+}^{p, p} V^{\prime}$ and $\Lambda_{+, w}^{p, p} V^{\prime}$ the spaces of Lagerberg $(p, p)$-forms that are strongly positive forms, positive and weakly positive respectively.

Remark 2.2.2 Strongly positive, positive or weakly positive Lagerberg forms and $\tau_{n}$ are symmetric. In the definition of weakly positive Lagerberg forms, it is necessary to impose the symmetry because the real analogue of Lemma 2.1.4 is not true, see Example 2.3.6.

It follows from Corollary 2.2.5 below that our definition of positive Lagerberg forms agrees with the definition given by Lagerberg in [16, Definition 2.1].

From our definition, we deduce that the product of positive Lagerberg forms is positive.

Definition 2.2.3 To $\eta \in \Lambda^{p, p} V^{\prime}$, we associate a bilinear form $|\eta|$ on $\Lambda^{p} V$ by the rule

$$
|\eta|(x, y)=(-1)^{\frac{p(p-1)}{2}} \eta(x, J(y)) .
$$

Moreover, for $q=n-p$, there is a duality pairing

$$
\langle\cdot, \cdot\rangle: \Lambda^{p, p} V^{\prime} \otimes \Lambda^{q, q} V^{\prime} \longrightarrow \mathbb{R},
$$

defined by $\eta \wedge \omega=\langle\eta, \omega\rangle \tau_{n}$ for $\eta \in \Lambda^{p, p} V^{\prime}$ and $\omega \in \Lambda^{q, q} V^{\prime}$.

Note that, again, the assignment $\eta \mapsto|\eta|$ is canonical and gives an isomorphism between $\Lambda^{p, p} V^{\prime}$ and the space of bilinear forms on $\Lambda^{p} V$. On the other hand, the duality pairing $\langle\cdot, \cdot\rangle$ depends on the choice of a basis, but only up to a positive number.

Proposition 2.2.4 A form $\eta \in \Lambda^{p, p} V^{\prime}$ is symmetric if and only if the bilinear form $|\eta|$ is symmetric. A form $\eta$ is positive if and only $|\eta|$ is a positive semidefinite symmetric form.

Proof The proof is similar to the complex case and is given in [16, Proposition 2.1].

Denote now by $\Lambda_{\text {sym }}^{p, p} V^{\prime}$ the subspace of symmetric elements and let $q:=n-p$. The duality pairing of Definition 2.2.3 induces a real duality pairing, denoted by the same symbol,

$$
\langle\cdot, \cdot\rangle: \Lambda_{\mathrm{sym}}^{p, p} V^{\prime} \otimes \Lambda_{\mathrm{sym}}^{q, q} V^{\prime} \rightarrow \mathbb{R}
$$

We give the analogue of Corollary 2.1.6 which was stated before [16, Lemma 2.2].

Corollary 2.2.5 For $p \in \mathbb{N}$, there are inclusions of closed convex cones

$$
\Lambda_{+, s}^{p, p} V^{\prime} \subset \Lambda_{+}^{p, p} V^{\prime} \subset \Lambda_{+, w}^{p, p} V^{\prime}
$$

in $\Lambda_{\mathrm{sym}}^{p, p} V^{\prime}$. For $q:=n-p$, the cones $\Lambda_{+, s}^{p, p} V^{\prime}$ and $\Lambda_{+, w}^{q, q} V^{\prime}$ are dual to each other and the cone $\Lambda_{+}^{p, p} V^{\prime}$ is the dual of $\Lambda_{+}^{q, q} V^{\prime}$ with respect to the above real duality pairing.

Proof The arguments are as in Corollary 2.1.6 replacing complex by real numbers and sesquilinearforms by symmetric bilinear forms.

Remark 2.2.6 As in the complex case, strong positivity agrees with positivity and weak positivity for $p=0,1, n-1, n$. Similarly as in [9, Example III 1.10], there are positive Lagerberg forms that are not strongly positive for any $2 \leq p \leq n-2$. 


\subsection{Comparison between the real and complex situations}

We aim for an identification of the space of Lagerberg forms $\Lambda^{\cdot \cdot} V^{\prime}$ with a subspace of the space of complex forms $\Lambda^{\cdot} \cdot V^{*}$ that preserves positivity as much as possible.

Definition 2.3.1 The vector space $V^{*}=\operatorname{Hom}_{\mathbb{R}}(V, \mathbb{C})$ has an antilinear involution $F$ coming from complex conjugation in $\mathbb{C}$. We extend $F$ to $V^{*} \oplus \bar{V}^{*}$ in such a way that $F$ and $\sigma$ (the complex conjugation from 2.1) anticommute:

$$
F(\bar{\alpha})=-\overline{F(\alpha)} \quad\left(\text { for } \alpha \in V^{*}\right) .
$$

There is a unique antilinear involution of the $\mathbb{R}$-algebra $\Lambda^{\cdot \cdot \cdot} V^{*}$ which extends $F$. We denote this extension also by $F$.

Note that $F$ induces an antilinear involution of any $\Lambda^{p, q} V^{*}$. In coordinates, we have

$$
F(\lambda)=\bar{\lambda}, \quad F\left(d u_{i}\right)=d u_{i} \quad \text { and } \quad F\left(d \bar{u}_{i}\right)=-d \bar{u}_{i}
$$

where $\lambda \in \Lambda^{0,0} V^{*}=\mathbb{C}$.

Note that, if $\eta \in \Lambda^{p, q} V^{*}$, then

$$
\overline{F(\eta)}=(-1)^{p+q} F(\bar{\eta}) .
$$

We see $V^{\prime} \subset V^{*}$ and $V^{\prime \prime} \subset \bar{V}^{*}$ as the subspaces of $F$-invariant elements. Note that $V^{\prime}$ looks like the space of real elements of $V^{*}$, while, due to the twisted definition (2.4) of $F$ in $\bar{V}^{*}$, the elements of $V^{\prime \prime}$ look like the imaginary elements of $\bar{V}^{*}$.

We extend the inclusion $V^{\prime} \oplus V^{\prime \prime} \rightarrow V^{*} \oplus \bar{V}^{*}$ to an $\mathbb{R}$-algebra homomorphism

$$
\Lambda^{\cdot, \cdot} V^{\prime} \longrightarrow \Lambda^{\cdot, \cdot} V^{*} \text {. }
$$

This inclusion sends $d^{\prime} u_{j}$ to $d u_{j}$ and $d^{\prime \prime} u_{j}$ to $i d \bar{u}_{j}$.

Proposition 2.3.2 We have the following compatibilities for the inclusion (2.7).

(i) The space of complex forms invariant under $F$ is the image of $\Lambda^{\prime}, \cdot V^{\prime}$.

(ii) The Lagerberg involution $J$ agrees on $V^{\prime} \oplus V^{\prime \prime}$ with the map $\alpha \mapsto i \bar{\alpha}$.

(iii) A Lagerberg $(p, p)$-form is symmetric if and only if it is real as a complex form.

(iv) The image of $\tau_{n}$ is $\omega_{n}$.

Proof Since $F$ is an antilinear involution, the space of $F$-invariant elements of $\Lambda^{, \cdot} V^{*}$ has real dimension $2^{2 n}$ which agrees with the dimension of $\Lambda^{\cdot}, V^{\prime}$. Since, by construction, the Lagerberg forms are invariant under $F$, both spaces agree. The remaining statements are direct computations.

Lemma 2.3.3 The involution F maps strongly positive (resp. weakly positive, resp. positive) complex forms to strongly positive (resp. weakly positive, resp. positive) complex forms.

Proof For any complex $(p, 0)$-form $\alpha$, antilinearity of $F$ and (2.6) give

$$
F\left(\alpha \wedge i^{p} \bar{\alpha}\right)=F \alpha \wedge(-i)^{p} F(\bar{\alpha})=F \alpha \wedge i^{p} \overline{F(\alpha)} .
$$

We conclude that $F$ preserves positivity of complex forms. Multiplicativity of $F$ and (2.8) show also that $F$ preserves strong positivity of forms.

Since $\omega_{n}$ is the image of $\tau_{n}$ under the above identification, it is fixed under $F$. Using that $F$ is an involution, we deduce from duality that $F$ preserves weak positivity as well. 
Proposition 2.3.4 The following compatibility conditions hold.

(i) A strongly positive Lagerberg form is also a strongly positive complex form.

(ii) An F-invariant weakly positive complex form is a weakly positive Lagerberg form.

This follows easily from the definitions. For positive forms, we have a stronger result.

Proposition 2.3.5 A Lagerberg form is positive if and only if it is positive as a complex form.

Proof It is easily seen that if $\omega \in \Lambda^{p, p} V^{\prime}$ is a positive Lagerberg form, then it is also a positive complex form by using the embedding (2.7) and that $J \omega=i^{p} \bar{\omega}$.

Conversely, assume that $\omega$ is a Lagerberg $(p, p)$-form that is positive as a complex form. We claim that is enough to show that if $\eta=(-1)^{\frac{p(p-1)}{2}} \alpha \wedge i^{p} \bar{\alpha}$ for some complex $(p, 0)$-form $\alpha$, then $\eta+F(\eta)$ is a positive Lagerberg form. Assuming this claim, we can write

$$
\omega=\sum_{s} \gamma_{s}(-1)^{\frac{p(p-1)}{2}} \alpha_{s} \wedge i^{p} \bar{\alpha}_{s}
$$

with $\gamma_{s} \in \mathbb{R}_{\geq 0}$ and $\alpha_{s} \in \Lambda^{p, 0} V^{*}$. Using the claim and the $F$-invariance of $\omega$, we have that

$$
\omega=\frac{1}{2}(\omega+F(\omega))=\sum_{s} \frac{\gamma_{s}}{2}(-1)^{\frac{p(p-1)}{2}}\left(\alpha_{s} \wedge i^{p} \bar{\alpha}_{s}+F\left(\alpha_{s} \wedge i^{p} \bar{\alpha}_{s}\right)\right)
$$

is a positive Lagerberg form.

To prove our claim, we write $\alpha=a+i b$ with $a, b \in \Lambda^{p, 0}\left(V^{\prime}\right)$. Then we have

$$
\alpha \wedge i^{p} \bar{\alpha}=(a+i b) \wedge i^{p}(\bar{a}-i \bar{b})=i^{p}(a \wedge \bar{a}-i a \wedge \bar{b}+i b \wedge \bar{a}+b \wedge \bar{b}) .
$$

Using $F(\alpha)=a-i b$, we get

$$
\left.F \alpha \wedge F\left(i^{p} \bar{\alpha}\right)\right)=(a-i b) \wedge F\left(i^{p}(\bar{a}-i \bar{b})\right)=i^{p}(a-i b) \wedge(\bar{a}+i \bar{b}),
$$

where we have used for the last equality that

$$
F\left(i^{p} \bar{a}\right)=(-1)^{p} i^{p} F(\bar{a})=(-1)^{p} i^{p}(-1)^{p} \overline{F(a)}=i^{p} \bar{a}
$$

by equation (2.6) and analogously $F\left(i^{p+1} \bar{b}\right)=-i^{p+1} \bar{b}$. Since

$$
\left.\frac{1}{2}(\eta+F(\eta))=(-1)^{\frac{p(p-1)}{2}} \frac{1}{2}\left(\alpha \wedge i^{p} \bar{\alpha}+F \alpha \wedge F\left(i^{p} \bar{\alpha}\right)\right)\right),
$$

we deduce that

$$
\eta+F(\eta)=2 \cdot(-1)^{\frac{p(p-1)}{2}} i^{p}(a \wedge \bar{a}+b \wedge \bar{b})=2 \cdot(-1)^{\frac{p(p-1)}{2}}(a \wedge J a+b \wedge J b)
$$

which is a positive Lagerberg form.

The next example shows that the analogues of Proposition 2.3.5 for strongly and weakly positive forms do not hold. Hence the converse of Proposition 2.3.4 (i) and (ii) is wrong.

Example 2.3.6 We show that the space of strongly positive Lagerberg forms does not span the space of symmetric Lagerberg forms. In particular, the cone of strongly positive Lagerberg forms has empty interior and not every symmetric Lagerberg form can be written as a difference of strongly positive ones.

Indeed, let $V$ be a real vector space of dimension four. Then we have

$$
\Lambda_{\mathrm{sym}}^{2,2} V^{\prime}=\left\{\sum_{i<k, j<l} w_{i j k l} d^{\prime} u_{i} \wedge d^{\prime \prime} u_{j} \wedge d^{\prime} u_{k} \wedge d^{\prime \prime} u_{l} \mid w_{i j k l}=w_{j i l k}\right\} .
$$


Moreover, we have the following identity of cones:

$$
\Lambda_{+, s}^{2,2} V^{\prime}=\left\langle a \wedge J(a) \wedge b \wedge J(b) \mid a, b \in \Lambda^{1,0} V^{\prime}\right\rangle_{\mathbb{R}_{\geq 0}} .
$$

Given $a, b \in \Lambda^{1,0} V^{\prime}$, let $w_{i j k l}(a, b) \in \mathbb{R}$ be such that

$$
a \wedge J(a) \wedge b \wedge J(b)=\sum_{i<k, j<l} w_{i j k l}(a, b) d^{\prime} u_{i} \wedge d^{\prime \prime} u_{j} \wedge d^{\prime} u_{k} \wedge d^{\prime \prime} u_{l}
$$

A direct computation shows

$$
w_{1,3,2,4}(a, b)-w_{1,2,3,4}(a, b)+w_{1,2,4,3}(a, b)=0,
$$

and, by symmetry, we have

$$
w_{3,1,4,2}(a, b)-w_{2,1,4,3}(a, b)+w_{2,1,3,4}(a, b)=0,
$$

Hence $\Lambda_{+, s}^{2,2} V^{\prime}$ is in a proper linear subspace of $\Lambda_{\mathrm{sym}}^{2,2} V^{\prime}$ and so it has empty interior.

As a consequence, the cone $\Lambda_{+, w}^{2,2} V^{\prime}$ is not strictly convex. Namely, the form

$$
\begin{aligned}
\omega= & d^{\prime} u_{3} \wedge d^{\prime \prime} u_{1} \wedge d^{\prime} u_{4} \wedge d^{\prime \prime} u_{2}-d^{\prime} u_{2} \wedge d^{\prime \prime} u_{1} \wedge d^{\prime} u_{4} \wedge d^{\prime \prime} u_{3} \\
& +d^{\prime} u_{2} \wedge d^{\prime \prime} u_{1} \wedge d^{\prime} u_{3} \wedge d^{\prime \prime} u_{4}+d^{\prime} u_{1} \wedge d^{\prime \prime} u_{3} \wedge d^{\prime} u_{2} \wedge d^{\prime \prime} u_{4} \\
& -d^{\prime} u_{1} \wedge d^{\prime \prime} u_{2} \wedge d^{\prime} u_{3} \wedge d^{\prime \prime} u_{4}+d^{\prime} u_{1} \wedge d^{\prime \prime} u_{2} \wedge d^{\prime} u_{4} \wedge d^{\prime \prime} u_{3}
\end{aligned}
$$

satisfies $\eta \wedge \omega=0$ for every $\eta \in \Lambda_{+, s}^{2,2} V^{\prime}$, and hence $C \omega \in \Lambda_{+, w}^{2,2} V^{\prime}$ for every $C \in \mathbb{R}$.

Remark 2.3.7 We will deduce from Example 2.3.6 the existence of Lagerberg forms that are strongly positive as complex forms but not strongly positive as Lagerberg forms.

By Lemma 2.1.4, every complex form $\omega$ can be written as a complex linear combination

$$
\omega=\sum_{i} \lambda_{i} \omega_{i}
$$

of strongly positive complex forms $\omega_{i}$. If $\omega$ is real, then applying $\sigma$ to (2.9) and using that the $\omega_{i}$ are real, we see that we may take the $\lambda_{i}$ to be real. If $\omega$ is further invariant under $F$, then applying $F$ to (2.9) we see that replacing $\omega_{i}$ by $1 / 2\left(\omega_{i}+F\left(\omega_{i}\right)\right)$ we may assume the $\omega_{i}$ to be $F$-invariant. Note that $F\left(\omega_{i}\right)$ is strongly positive by Lemma 2.3.3.

We have just shown $\Lambda_{\mathrm{sym}}^{p, p} V^{\prime}=\left\langle\left(\Lambda_{+, s}^{p, p} V^{*}\right)^{F}\right\rangle_{\mathbb{R}}$. Since we showed in Example 2.3.6 that $\left\langle\Lambda_{+, s}^{p, p} V^{\prime}\right\rangle_{\mathbb{R}} \subsetneq \Lambda_{\mathrm{sym}}^{p, p} V^{\prime}$, we find that $\left(\Lambda_{+, s}^{p, p} V^{*}\right)^{F} \not \subset \Lambda_{+, s}^{p, p} V^{\prime}$. By Proposition 2.3.2, this means exactly that not every Lagerberg form that is strongly positive as a complex form is strongly positive as a Lagerberg form.

The next example illustrates this phenomenon.

Example 2.3.8 Let $V$ still be a real vector space of dimension four. Choose a basis $e_{1}, \ldots, e_{4}$ and corresponding bases of $V^{*}, \bar{V}^{*}, V^{\prime}$ and $V^{\prime \prime}$ as before. The complex form

$$
\eta=\left(d u_{1}+i d u_{2}\right) \wedge i\left(d \bar{u}_{1}-i d \bar{u}_{2}\right) \wedge\left(d u_{3}+i d u_{4}\right) \wedge i\left(d \bar{u}_{3}-i d \bar{u}_{4}\right)
$$

is strongly positive by definition. By Lemma 2.3.3, the form $\omega=\frac{1}{2}(\eta+F(\eta))$ is strongly positive. Moreover, it is $F$-invariant and hence $\omega$ may be seen as a Lagerberg form by Proposition 2.3.2. We claim that $\omega$ is not strongly positive as a Lagerberg form. 
A direct computation shows that

$$
\begin{aligned}
\omega= & d^{\prime} u_{1} \wedge d^{\prime \prime} u_{1} \wedge d^{\prime} u_{3} \wedge d^{\prime \prime} u_{3}+d^{\prime} u_{1} \wedge d^{\prime \prime} u_{1} \wedge d^{\prime} u_{4} \wedge d^{\prime \prime} u_{4} \\
& +d^{\prime} u_{2} \wedge d^{\prime \prime} u_{2} \wedge d^{\prime} u_{3} \wedge d^{\prime \prime} u_{3}+d^{\prime} u_{2} \wedge d^{\prime \prime} u_{2} \wedge d^{\prime} u_{4} \wedge d^{\prime \prime} u_{4} \\
& -d^{\prime} u_{1} \wedge d^{\prime \prime} u_{2} \wedge d^{\prime} u_{3} \wedge d^{\prime \prime} u_{4}+d^{\prime} u_{2} \wedge d^{\prime \prime} u_{1} \wedge d^{\prime} u_{3} \wedge d^{\prime \prime} u_{4} \\
& +d^{\prime} u_{1} \wedge d^{\prime \prime} u_{2} \wedge d^{\prime} u_{4} \wedge d^{\prime \prime} u_{3}-d^{\prime} u_{2} \wedge d^{\prime \prime} u_{1} \wedge d^{\prime} u_{4} \wedge d^{\prime \prime} u_{3} \\
= & (-1)\left(d^{\prime} u_{1} \wedge d^{\prime} u_{3}-d^{\prime} u_{2} \wedge d^{\prime} u_{4}\right) \wedge J\left(d^{\prime} u_{1} \wedge d^{\prime} u_{3}-d^{\prime} u_{2} \wedge d^{\prime} u_{4}\right) \\
& +(-1)\left(d^{\prime} u_{1} \wedge d^{\prime} u_{4}+d^{\prime} u_{2} \wedge d^{\prime} u_{3}\right) \wedge J\left(d^{\prime} u_{1} \wedge d^{\prime} u_{4}+d^{\prime} u_{2} \wedge d^{\prime} u_{3}\right)
\end{aligned}
$$

This shows that $\omega$ is a positive Lagerberg form, that the associated symmetric bilinear form $|\omega|$ has rank 2 and that $(\operatorname{ker}|\omega|)^{\perp}$ is the 2-dimensional subspace

$$
(\operatorname{ker}|\omega|)^{\perp}=\mathbb{R}\left(d^{\prime} u_{1} \wedge d^{\prime} u_{3}-d^{\prime} u_{2} \wedge d^{\prime} u_{4}\right)+\mathbb{R}\left(d^{\prime} u_{1} \wedge d^{\prime} u_{4}+d^{\prime} u_{2} \wedge d^{\prime} u_{3}\right) .
$$

We pick any decomposition

$$
\omega=\sum_{j} \alpha_{j} \wedge J\left(\alpha_{j}\right)
$$

with $\alpha \in \Lambda^{2,0} V^{\prime}$. Then $\alpha_{j} \in(\operatorname{ker}|\omega|)^{\perp}$, since for any $v \in \operatorname{ker}|\omega| \subset \Lambda^{2} V$, we have

$$
0=|\omega|(v, v)=\sum_{j}\left\langle\alpha_{j}, v\right\rangle^{2} .
$$

If $\omega$ were strongly positive, we would have a decomposition like (2.10) where $\alpha_{j} \in \Lambda^{2,0} V^{\prime}$ is a product of $(1,0)$-forms. However, this is not possible because $(\mathrm{ker}|\omega|)^{\perp}$ does not contain any non-zero real decomposable element as the following argument shows. Assume that

$$
\rho:=\left(\alpha d^{\prime} u_{1}+\beta d^{\prime} u_{2}+\gamma d^{\prime} u_{3}+\delta d^{\prime} u_{4}\right) \wedge\left(\alpha^{\prime} d^{\prime} u_{1}+\beta^{\prime} d^{\prime} u_{2}+\gamma^{\prime} d^{\prime} u_{3}+\delta^{\prime} d^{\prime} u_{4}\right)
$$

belongs to $(\operatorname{ker}|\omega|)^{\perp}$.

This implies the equations

$$
\begin{aligned}
\alpha \gamma^{\prime}-\alpha^{\prime} \gamma & =\beta^{\prime} \delta-\beta \delta^{\prime}, \\
\alpha \delta^{\prime}-\alpha^{\prime} \delta & =\beta \gamma^{\prime}-\beta^{\prime} \gamma, \\
\alpha \beta^{\prime}-\alpha^{\prime} \beta & =0, \\
\gamma \delta^{\prime}-\gamma^{\prime} \delta & =0 .
\end{aligned}
$$

The point $\rho$ determines a point $\rho^{\prime}$ in the Grassmannian $\operatorname{Gr}(2,4)$ with Plücker coordinates

$$
\begin{array}{ll}
x=\left|\begin{array}{cc}
\alpha & \beta \\
\alpha^{\prime} & \beta^{\prime}
\end{array}\right|, \quad y=\left|\begin{array}{cc}
\alpha & \gamma \\
\alpha^{\prime} & \gamma^{\prime}
\end{array}\right|, \quad z=\left|\begin{array}{cc}
\alpha & \delta \\
\alpha^{\prime} & \delta^{\prime}
\end{array}\right|, \\
u=\left|\begin{array}{cc}
\beta & \gamma \\
\beta^{\prime} & \gamma^{\prime}
\end{array}\right|, \quad v=\left|\begin{array}{cc}
\beta & \delta \\
\beta^{\prime} & \delta^{\prime}
\end{array}\right|, \quad w=\left|\begin{array}{cc}
\gamma & \delta \\
\gamma^{\prime} & \delta^{\prime}
\end{array}\right| .
\end{array}
$$

The previous equations imply that the Plücker coordinates of $\rho^{\prime}$ satisfy the equations

$$
y=-v, \quad z=u, \quad x=w=0 .
$$

Moreover, the Plücker equations for $\operatorname{Gr}(2,4)$ are reduced to the single equation

$$
x w-y v+z u=0 .
$$


We conclude that the Plücker coordinates of $\rho^{\prime}$ satisfy the equation

$$
y^{2}+z^{2}=0
$$

that has no real solutions except the trivial one. The fact that $\omega$ is strongly positive as a complex form is reflected by the fact that (2.11) has non-trivial complex solutions.

\section{Lagerberg forms and Lagerberg currents on partial compactifications}

For convex geometry we will use the notation and conventions set up in [12, Appendix]. Let $N$ be a free abelian group of $\operatorname{rank} n, M=\operatorname{Hom}_{\mathbb{Z}}(N, \mathbb{Z})$ its dual and denote by $N_{\mathbb{R}}$ resp. $M_{\mathbb{R}}$ the respective scalar extensions to $\mathbb{R}$.

\subsection{Partial compactifications}

A strictly convex rational polyhedral cone $\sigma \in N_{\mathbb{R}}$ is a polyhedron defined by finitely many equations of the form $\varphi() \geq$.0 with $\varphi \in M$, that does not contain a positive dimensional linear subspace. A rational polyhedral fan $\Sigma$ in $N_{\mathbb{R}}$ is a polyhedral complex all of whose polyhedra are strictly convex rational cones. In this paper we make the convention that a fan is always a rational polyhedral fan.

A cone is called smooth if it is generated by a subset of a $\mathbb{Z}$-basis of $N$. A fan $\Sigma$ is called smooth if each cone of $\Sigma$ is smooth. For $\sigma \in \Sigma$ we define the monoid $S_{\sigma}:=\{\varphi \in$ $M \mid \varphi(v) \geq 0$ for all $v \in \sigma\}$. For $\sigma \in \Sigma$, write $N(\sigma):=N_{\mathbb{R}} /\langle\sigma\rangle_{\mathbb{R}}$ where $\langle\sigma\rangle_{\mathbb{R}}$ denotes the real vector space generated by $\sigma$. Given $\sigma, \tau \in \Sigma$, we write $\tau \prec \sigma$ if $\tau$ is a face of $\sigma$. We have projection maps $\pi_{\sigma}: N_{\mathbb{R}} \rightarrow N(\sigma)$ and $\pi_{\sigma, \tau}: N(\tau) \rightarrow N(\sigma)$ for $\tau \prec \sigma$.

Definition 3.1.1 Let $\Sigma \subset N_{\mathbb{R}}$ be a rational polyhedral fan. We consider the disjoint union

$$
N_{\Sigma}:=\coprod_{\sigma \in \Sigma} N(\sigma)
$$

and call $N_{\Sigma}$ equipped with the topology introduced in Remark 3.1.2 the partial compactification of $N_{\mathbb{R}}$ associated to $\Sigma$.

Remark 3.1.2 The partial compactification $N_{\Sigma}$ carries the following topology which is Hausdorff. It is also locally compact and has a countable basis and hence it is metrizable. Let us briefly recall its definition.

First, we define the partial compactification of $N(\sigma)$ for a single cone $\sigma \in \Sigma$ by setting

$$
N_{\sigma}:=\coprod_{\tau \prec \sigma} N(\tau)
$$

The set $N_{\sigma}$ is naturally identified with the monoid morphisms $\operatorname{Hom}$ Mon $\left(S_{\sigma}, \mathbb{R}_{\infty}\right)$. We equip it with the subspace topology of $\mathbb{R}_{\infty}^{S_{\sigma}}$. Using a finite set of generators $\varphi_{1}, \ldots, \varphi_{k}$ for the monoid $S_{\sigma}$, we can realize $\operatorname{Hom}_{\mathbf{M o n}}\left(S_{\sigma}, \mathbb{R}_{\infty}\right)$ as a closed subspace of $\mathbb{R}_{\infty}^{k}$ with the induced topology (see [18, Remark 3.1] and use [18, Lemma 2.1]).

For a face $\rho$ of $\sigma$, we note that $N_{\rho}$ is an open subset of $N_{\sigma}$. This is used to define a topology on the partial compactification $N_{\Sigma}$ by gluing the partial compactifications $N_{\sigma}, \sigma \in \Sigma$, along the open subsets induced by common faces.

We give a second description of the topology of $N_{\Sigma}$. To this end, we fix an Euclidean metric in $N_{\mathbb{R}}$. For a cone $v$ of $\Sigma$, the Euclidean metric allows us to identify $v^{\perp}$ with a subspace 
of $N_{\mathbb{R}}$ and, through the projection $\pi_{v}$, with the space $N(v)$. Again, we consider first the case of a single cone $\sigma \in \Sigma$. For a point $u \in N_{\sigma}$, there is a unique face $v$ of $\sigma$ with $u \in N(v)$. Let $u_{0} \in v^{\perp} \subset N_{\mathbb{R}}$ be the corresponding point and let $U$ be a neighborhood of $u_{0}$ in $v^{\perp}$. For each face $\tau \prec v$, the cone $v$ induces a cone $\pi_{\tau}(v)$ contained in $N(\tau)$. For each $p \in v$, we write

$$
W(v, U, p)=\coprod_{\tau \prec v} \pi_{\tau}(U+p+v) .
$$

The topology of $N_{\sigma}$ is defined by the fact that $\{W(v, U, p)\}_{U, p}$ is a basis of neighbourhoods of $u$ in $N_{\sigma}$ for any $u \in N_{\sigma}$. As before the topology of $N_{\Sigma}$ is defined by gluing along the open subsets $N_{\tau}$ of $N_{\sigma}$ whenever $\tau \prec \sigma$.

The first definition of the topology is given by Kajiwara [15] and by Payne [18], the second definition is from [1, I.1]. The topologies coincide as the above basis of neighbourhoods works also for the first definition by [18, Remark 3.4].

To prove that $N_{\Sigma}$ is Hausdorff, we use that the quotient of a topological space (in this case the disjoint union of the $N_{\sigma}$ ) by an equivalence relation is Hausdorff if the canonical map to the quotient is open and the graph of the equivalence relation is closed [5, Ch. I $\$ 8.3$ Prop. 8]. As the map to the quotient is open by construction, it is enough to show that, for cones $\sigma_{1}$ and $\sigma_{2}$ with $\tau=\sigma_{1} \cap \sigma_{2}$, the map

$$
N_{\tau} \longrightarrow N_{\sigma_{1}} \times N_{\sigma_{2}}
$$

is a closed immersion. This follows easily from the first description of the topologies of $N_{\sigma_{1}}$ and $N_{\sigma_{2}}$ by choosing a finite set of generators of $S_{\sigma_{1}}$ and $S_{\sigma_{2}}$ and observing that the union of both sets is a set of generators of $S_{\tau}$.

Note that $N_{\Sigma}$ is locally compact because the $N_{\sigma}$ provide an open covering of $N_{\Sigma}$ and each of them is locally compact. Finally every $N_{\sigma}$ has a countable basis and hence also $N_{\Sigma}$.

Remark 3.1.3 Let $\mathbb{T}=$ Spec $\mathbb{C}[M]$ be the split complex torus with cocharacter lattice $N$. Let $X_{\Sigma}$ denote the toric variety over $\mathbb{C}$ with dense torus $\mathbb{T}$ determined by the fan $\Sigma$ in $N_{\mathbb{R}}$. Let $X_{\Sigma}^{\text {an }}$ denote the analytification of $X_{\Sigma}$, i.e. the set of complex points $X_{\Sigma}(\mathbb{C})$ with its structure of an analytic space.

There is a well-known continuous map (see for example [1, I.1, p.2], [15, Definition 1.2] or $[6$, Section 4.1])

$$
\text { trop: } X_{\Sigma}^{\text {an }} \longrightarrow N_{\Sigma}
$$

which is nowadays called tropicalization map as it is given by glueing on the affine open subsets $U_{\sigma}=\operatorname{Spec} \mathbb{C}\left[S_{\sigma}\right]$ for $\sigma \in \Sigma$ of $X_{\Sigma}$ the tropicalization maps

$$
\text { trop: } U_{\sigma}^{\text {an }} \longrightarrow N_{\sigma}=\operatorname{Hom}_{\text {Mon }}\left(S_{\sigma}, \mathbb{R}_{\infty}\right), y \longmapsto\left(m \longmapsto-\log \left|\chi^{m}(y)\right|\right)
$$

where $\chi^{m}: \mathbb{T} \rightarrow \mathbb{G}_{m}$ is the character associated with $m$. It follows from [6, Sections 4.1, 4.2] that the tropicalization map (3.2) is a proper continuous map that identifies $N_{\Sigma}$ with $X_{\Sigma}^{\text {an }} / \mathbb{S}$ where $\mathbb{S}$ denotes the real compact torus

$$
\mathbb{S}=\left\{p \in \mathbb{T}^{\text {an }}|| \chi^{m}(p) \mid=1 \text { for all } m \in M\right\} \subset \mathbb{T}^{\text {an }} .
$$

There is a continuous proper section $\rho_{\Sigma}: N_{\Sigma} \rightarrow X_{\Sigma}^{\mathrm{an}}$ of the tropicalization map (3.2) given as the unique continuous extension of the section

$$
\rho: N_{\mathbb{R}} \longrightarrow \mathbb{T}^{\text {an }}=\operatorname{Hom}\left(M, \mathbb{C}^{\times}\right), n \longmapsto[m \longmapsto \exp (-\langle m, n\rangle)]
$$

of (3.2) (see [6, Remark 4.1.3]). 

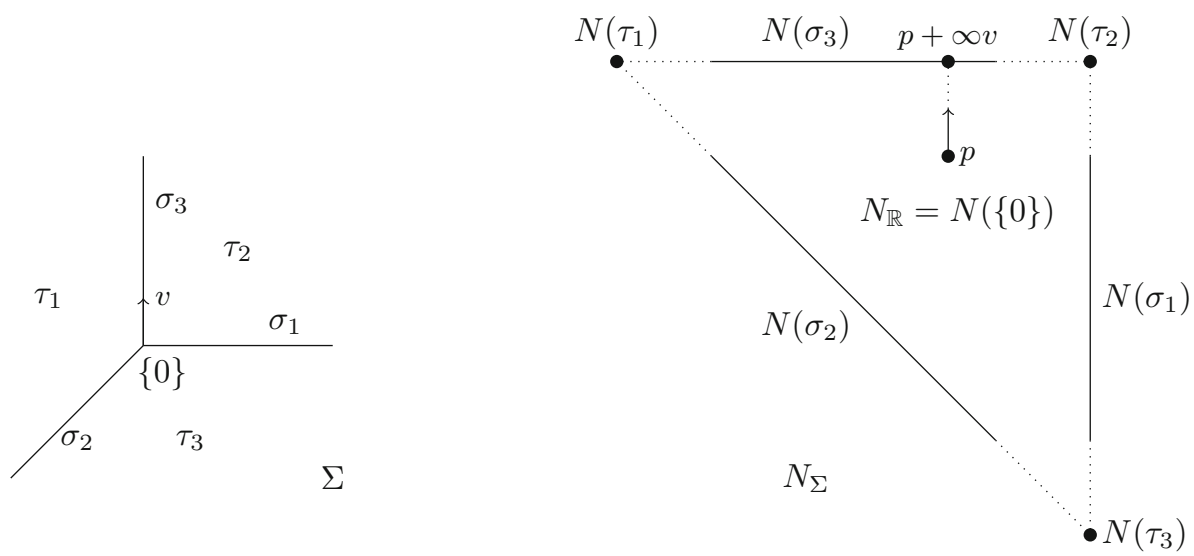

Fig. 1 The fan and partial compactification of the toric variety $\mathbb{P}^{2}$

To illustrate the topology on the partial compactification $N_{\Sigma}$, we give the following lemma where the notation coincides with the one in $[1, \mathrm{I} .1, \mathrm{p} .5]$.

Lemma 3.1.4 Let $\Sigma \subset N_{\mathbb{R}}$ be a fan, and let $N_{\Sigma}$ be its associated partial compactification. Given $p \in N_{\mathbb{R}}$ and $v \in|\Sigma|$, the limit $p+\infty v:=\lim _{\mu \rightarrow+\infty} p+\mu v$ exists in $N_{\Sigma}$. Moreover, $p+\infty v \in N(\sigma)$ for the unique cone $\sigma \in \Sigma$ such that $v \in \operatorname{relint}(\sigma)$.

Proof We use the description of the topology of $N_{\Sigma}$ given by the basis of neighborhoods $W(\sigma, U, q)$, so we fix an Euclidean metric in $N_{\mathbb{R}}$. Let $p_{0} \in \sigma^{\perp}$ be the point corresponding to $\pi_{\sigma}(p), U$ a neighborhood of $p_{0}$ in $\sigma^{\perp}$ and $q \in \sigma$. It is enough to show that there is a $\mu_{0}>0$ and for all $\mu \geq \mu_{0}$, the condition $p+\mu v \in W(\sigma, U, q)$ holds. Since $\pi_{\sigma}(p)=\pi_{\sigma}\left(p_{0}\right)$ and $q \in \sigma$, we deduce that $p-p_{0}-q \in\langle\sigma\rangle_{\mathbb{R}}$. Since $v \in \operatorname{relint}(\sigma)$, there is a $\mu_{0}$ such that for all $\mu \geq \mu_{0}$, we have $p-p_{0}-q+\mu v \in \sigma$ and hence $p+\mu v \in q+U+\sigma=W(\sigma, U, q)$.

Example 3.1.5 As an example, we consider the toric variety $\mathbb{P}^{2}$ and its tropicalization shown in Fig. 1. Note that for $v=(0,1)$, the point $p+\infty v$ is the point in $N\left(\sigma_{3}\right)$ lying vertically above $p$.

\subsection{Lagerberg forms and Lagerberg currents}

Recall from [16] that for every open subset $U$ of $N_{\mathbb{R}}$, there is a bigraded $\mathbb{R}$-algebra of Lagerberg forms $A^{\cdot, \cdot}(U)$ with differentials $d^{\prime}$ and $d^{\prime \prime}$ of bidegree $(1,0)$ and $(0,1)$. Lagerberg forms were introduced by Lagerberg in loc. cit. under the name superforms. They are defined as

$$
A^{p, q}(U)=A^{p}(U) \otimes_{C^{\infty}(U)} A^{q}(U)
$$

where $A^{\cdot}(U)$ denotes the $\mathbb{R}$-algebra of real valued smooth differential forms on $U$.

We choose a basis of $N$ which defines coordinates $u_{1}, \ldots, u_{n}$ on $N_{\mathbb{R}}$. Then we may write a Lagerberg form $\alpha$ as

$$
\alpha=\sum_{I, J} f_{I J} d^{\prime} u_{I} \wedge d^{\prime \prime} u_{J}
$$


where $I=\left\{i_{1}<\cdots<i_{p}\right\}$ and $J=\left\{j_{1}<\cdots<j_{q}\right\}$ range over all subsets of $\{1, \ldots, n\}$, where $f_{I J}$ are smooth real functions on $U$ and we use the multi-index notation

$$
d^{\prime} u_{I} \wedge d^{\prime \prime} u_{J}:=d u_{i_{1}} \wedge \cdots \wedge d u_{i_{p}} \otimes d u_{j_{1}} \wedge \cdots \wedge d u_{j_{q}} .
$$

There are differentials $d^{\prime}: A^{p, q}(U) \rightarrow A^{p+1, q}(U)$ and $d^{\prime \prime}: A^{p, q}(U) \rightarrow A^{p, q+1}(U)$, which are in coordinates given by

$$
d^{\prime}\left(f d^{\prime} u_{I} \wedge d^{\prime \prime} u_{J}\right)=\sum_{i_{0}=1}^{n} \frac{\partial f}{\partial u_{i_{0}}} d^{\prime} u_{i_{0}} \wedge d^{\prime} u_{I} \wedge d^{\prime \prime} u_{J}
$$

and

$$
d^{\prime \prime}\left(f d^{\prime} u_{I} \wedge d^{\prime \prime} u_{J}\right)=(-1)^{p} \sum_{j_{0}=1}^{n} \frac{\partial f}{\partial u_{j_{0}}} d^{\prime} u_{I} \wedge d^{\prime \prime} u_{j_{0}} \wedge d^{\prime \prime} u_{J}
$$

The product of the bigraded $\mathbb{R}$-algebra $A^{\cdot, \cdot}(U)$ is alternating and we denote it by $\wedge$. The algebras $A^{\cdot, \cdot}(U)$ form a sheaf on $N_{\mathbb{R}}$ that is denoted by $A^{\cdot, \cdot}$ or by $A_{N_{\mathbb{R}}}^{\cdot}$.

The algebra $A^{\cdot, \cdot}(U)$ carries a natural involution $J$ that permutes bidegrees and is determined by $J(\alpha \otimes \beta)=\beta \otimes \alpha$ for all $\alpha, \beta \in A^{\cdot}(U)$. A Lagerberg form $\alpha \in A^{p, p}(U)$ of type $(p, p)$ is called symmetric if it satisfies $J(\alpha)=(-1)^{p} \alpha$.

Let us fix a fan $\Sigma \subset N_{\mathbb{R}}$. Following [14, Definition 2.4], smooth forms on open subsets of $N_{\Sigma}$ are defined as follows.

Definition 3.2.1 Let $U \subset N_{\Sigma}$ be an open subset. For every $\sigma \in \Sigma$, we write $U_{\sigma}:=U \cap N(\sigma)$. A Lagerberg form of type $(p, q)$ on $U$ is given by a family $\omega=\left(\omega_{\sigma}\right)_{\sigma \in \Sigma}$ with $\omega_{\sigma} \in A^{p, q}\left(U_{\sigma}\right)$ satisfying the following local condition. For each $p \in U_{\sigma}$, there exists a neighborhood $V$ of $p$ in $U$ such that for all $\tau \prec \sigma$ we have

$$
\left.\omega_{\tau}\right|_{V_{\tau} \cap \pi_{\sigma, \tau}^{-1}\left(V_{\sigma}\right)}=\left.\pi_{\sigma, \tau}^{*}\left(\left.\omega_{\sigma}\right|_{V_{\sigma}}\right)\right|_{V_{\tau} \cap \pi_{\sigma, \tau}^{-1}\left(V_{\sigma}\right)} .
$$

We denote by $A^{p, q}(U)$ the real vector space of Lagerberg forms of type $(p, q)$ on $U$. There are unique differentials $d^{\prime}: A^{p, q}(U) \rightarrow A^{p+1, q}(U)$ and $d^{\prime \prime}: A^{p, q}(U) \rightarrow A^{p, q+1}(U)$ such that $\left(d^{\prime} \omega\right)_{\sigma}=d^{\prime}\left(\omega_{\sigma}\right)$ and $\left(d^{\prime \prime} \omega\right)_{\sigma}=d^{\prime \prime}\left(\omega_{\sigma}\right)$ for each $\omega \in A^{p, q}(U)$ and each $\sigma \in \Sigma$. A smooth function $f: U \rightarrow \mathbb{R}$ is a Lagerberg form of type $(0,0)$ in $U$. The assignment $U \mapsto A^{p, q}(U)$ defines a sheaf $A^{p, q}$ of real vector spaces on the topological space $N_{\Sigma}$. If we want to stress the fact that $A^{p, q}$ is a sheaf on $N_{\Sigma}$, we will denote it by $A_{N_{\Sigma}}^{p, q}$, The support $\operatorname{supp}(\omega)$ of a Lagerberg form $\omega \in A^{p, q}(U)$ is the closed subset of points of $U$ where $\omega$ has a non-zero germ in the stalk. The space of Lagerberg forms of type $(p, q)$ on an open subset $U$ of $N_{\Sigma}$ with compact support is denoted $A_{c}^{p, q}(U)$.

Remark 3.2.2 The stalk $A_{N_{\Sigma}, x}^{p, q}$ of the sheaf $A_{N_{\Sigma}}^{p, q}$ in a point $x \in N(\sigma) \subset N_{\Sigma}$ can be identified with the stalk $A_{N(\sigma), x}^{p, q}$ in $x$ of the sheaf of Lagerberg forms on the real vector space $N(\sigma)$. This follows from Definition 3.2.1.

(ii) Let $U \subset N_{\Sigma}$ be an open subset and $\omega \in A^{p, q}(U)$ a Lagerberg form. It follows from statement (i) that $\operatorname{supp}(\omega)=\cup_{\sigma \in \Sigma} \operatorname{supp}\left(\omega_{\sigma}\right)$, see [14, Lemma 2.17].

(iii) Let $U \subset N_{\Sigma}$ be an open subset. There is an involution $J$ on

$$
A^{\cdot \cdot \cdot}(U)=\oplus_{p, q \geq 0} A^{p, q}(U)
$$

such that given a Lagerberg form $\omega=\left(\omega_{\sigma}\right)_{\omega \in \Sigma}$ as in Definition 3.2.1, $J(\omega)$ is determined by $(J \omega)_{\sigma}=J\left(\omega_{\sigma}\right)$. A Lagerberg form $\omega \in A^{p, p}(U)$ of type $(p, p)$ is called symmetric if it 
satisfies $J(\omega)=(-1)^{p} \omega$.

(iv) For $U \subset N_{\Sigma}$ open, the restriction map $A^{p, q}(U) \rightarrow A^{p, q}\left(U \cap N_{\mathbb{R}}\right)$ is injective.

Remark 3.2.3 Let $U$ be an open subset of $N_{\Sigma}$. Observe that $A_{c}^{p, q}(U)$ is in general not a finitely generated $A_{c}^{0,0}(U)$-module. This is caused by the fact that forms of large degree vanish automatically at the boundary.

We next discuss a topology on the space $A_{c}^{p, q}(U)$. This topology is modeled on the topology of the space of test forms used in analysis, see for instance [22, §6]. In fact, for an open subset $U$ of $N_{\Sigma}$, we will define topologies on certain subspaces of $A_{c}^{p, q}(U)$ and use a limit process to define a topology on $A_{c}^{p, q}(U)$. Moreover, we shall describe the convergent sequences in $A_{c}^{p, q}(U)$. In the following, we fix a basis $u_{1}, \ldots, u_{n}$ of $M$ which defines coordinates $\left(u_{1}, \ldots, u_{n}\right): N_{\mathbb{R}} \stackrel{\sim}{\rightarrow} \mathbb{R}^{n}$ and allows to write Lagerberg forms on $U$ in terms of standard forms $d^{\prime} u_{I} \wedge d^{\prime \prime} u_{J}$ for subsets $I, J \subset\{1, \ldots, n\}$.

Definition 3.2.4 Let $U$ be an open subset of $N_{\Sigma}$. For each compact subset $K \subset U$ and each finite open covering $\left(V_{i}\right)_{i}$ of $K$, we denote by $A_{K}^{p, q}\left(U,\left(V_{i}\right)_{i}\right)$ the subset of all Lagerberg forms $\omega=\left(\omega_{\sigma}\right)_{\sigma \in \Sigma}$ in $A_{c}^{p, q}(U)$ such that $\operatorname{supp}(\omega) \subset K$ and

$$
\left.\omega_{\tau}\right|_{V_{i, \tau} \cap \pi_{\sigma, \tau}^{-1}\left(V_{i, \sigma}\right)}=\left.\pi_{\sigma, \tau}^{*}\left(\left.\omega_{\sigma}\right|_{V_{i, \sigma}}\right)\right|_{V_{i, \tau} \cap \pi_{\sigma, \tau}^{-1}\left(V_{i, \sigma}\right)}
$$

holds for all $i$ and all cones $\sigma, \tau \in \Sigma$ with $\tau \prec \sigma$ and $V_{i, \sigma}=V_{i} \cap N(\sigma) \neq \emptyset$. Given $\omega \in A_{K}^{p, q}\left(U,\left(V_{i}\right)_{i}\right)$, we write $\omega=\sum_{I, J \subset\{1, \ldots, n\}} f_{I, J} d^{\prime} u_{I} \wedge d^{\prime \prime} u_{J}$ and define

$$
\|\omega\|_{m}:=\sum_{\substack{\alpha \in \mathbb{N}^{n} \\|\alpha| \leq m}} \sum_{I, J \subset\{1, \ldots, n\}}\left\|\frac{\partial^{|\alpha|} f_{I, J}}{\partial u^{\alpha}}\right\|_{K}
$$

for each $m \in \mathbb{N}$ using the supremum norm \|\|$_{K}$ of continuous real functions on the compact set $K$. The family of norms (3.4), where $m \in \mathbb{N}$ varies, defines on $A_{K}^{p, q}\left(U,\left(V_{i}\right)_{i}\right)$ the structure of a locally convex topological vector space which is complete with respect to a translation invariant metric and hence it is a Fréchet space. The induced topology is denoted by $\tau_{K,\left(V_{i}\right)_{i}}$.

We put on $A_{c}^{p, q}(U)$ the topology $\tau$, defined as the limit topology

$$
\left(A_{c}^{p, q}(U), \tau\right)=\underset{K,\left(V_{i}\right)_{i}}{\lim _{K}}\left(A_{K}^{p, q}\left(U,\left(V_{i}\right)_{i}\right), \tau_{\left.K,\left(V_{i}\right)_{i}\right)}\right)
$$

in the category of locally convex topological vector spaces. Note that this may be different from the direct limit in the category of topological vector spaces.

As mentioned previously, the topology of $A_{c}^{p, q}(U)$ is modeled on the classical topology on the space of test functions in $[22, \S 6]$ and its formal properties are very similar. For instance, if $U$ is not compact, then $A_{c}^{p, q}(U)$ is not metrizable. Nevertheless the topology on $A_{c}^{p, q}(U)$ has many nice properties and the fact that is not metrizable is only a minor issue.

Remark 3.2.5 The spaces $A_{c}^{p, q}(U)$ have the same properties as the test function spaces in [22, Chapter 6]. This is a consequence from the fact that $E:=A_{c}^{p, q}(U)$ is an $L F$-space as introduced by Dieudonné and Schwartz [10]. This means that the vector space $E$ is a countable union of strictly increasing Fréchet spaces $E_{k}$ such that the topology on $E_{k}$ agrees with the induced topology from $E_{k+1}$. Indeed, using that $U$ has a countable basis, it is clear that the direct limit can be by described by using countable many $\left(K_{k},\left(V_{k, i}\right)_{i}\right)$ such that the 
compact subset $K_{k}$ lies in the interior of $K_{k+1}$ and such that a subfamily of $\left(V_{k+1, i}\right)_{i}$ is a refinement of the open covering $\left(V_{k, i}\right)_{i}$ of $K_{k}$. Setting

$$
E_{k}:=\left(A_{K}^{p, q}\left(U,\left(V_{i}\right)_{i}\right), \tau_{K,\left(V_{i}\right)_{i}}\right),
$$

we see that $E$ is an $L F$-space. It is shown in [10] that $E$ has a canonical structure as a locally convex space which is the finest structure such that the topology on $E_{n}$ agrees with the induced topology and it follows that $E$ is the direct limit of the $E_{n}$ in the category of locally convex spaces.

All properties of test function spaces from [22, Chapter 6] were shown in [10] more generally for $L F$-spaces and so they apply to $A_{c}^{p, q}(U)$. In fact, an $L F$-space is not only sequentially complete, but a complete Hausdorf space [10, Corollary of Theorem 6]. For our paper, we need mainly the following results about sequences.

Proposition 3.2.6 Let $U$ be an open subset of $N_{\Sigma}$. A sequence $\left(\omega_{k}\right)$ in $A_{c}^{p, q}(U)$ converges to $\omega \in A_{c}^{p, q}(U)$ if and only if there is a compact subset $K \subset U$ and a finite open covering $\left(V_{i}\right)_{i}$ of $K$ such that all $\omega_{k}$ and $\omega$ are contained in $A_{K}^{p, q}\left(U,\left(V_{i}\right)_{i}\right)$ and for every $m \in \mathbb{N}$, we have $\lim _{k \rightarrow \infty}\left\|\omega_{k}-\omega\right\|_{m}=0$.

Proof A convergent sequence is bounded and hence the result follows from the fact that every bounded subset of an $L F$-space is contained in some $E_{n}$ [10, Proposition 4].

Even if the space $A_{c}^{p, q}(U)$ is not metrizable, for many purposes, sequences are enough.

Proposition 3.2.7 Let $T: A_{c}^{n-p, n-q}(U) \rightarrow \mathbb{R}$ be a linear functional. Then the following conditions are equivalent.

(i) The map $T$ is continuous.

(ii) If a sequence $\left(\omega_{k}\right)_{k \in \mathbb{N}}$ converges to zero, then $\left(T\left(\omega_{k}\right)\right)_{k \in \mathbb{N}}$ converges to zero.

(iii) The restriction of $T$ to each subspace $A_{K}^{n-p, n-q}\left(U,\left(V_{i}\right)_{i}\right)$ is continuous.

Proof The equivalence of (i) and (iii) follows from [10, Proposition 5], while (ii) and (iii) are equivalent by Proposition 3.2.6.

As in the classical case of distributions, we now define currents as the topological dual of the space of smooth forms with compact support.

Definition 3.2.8 A Lagerberg current of type $(p, q)$ on $U$ is a continuous linear functional $T: A_{c}^{n-p, n-q}(U) \rightarrow \mathbb{R}$. The space of such Lagerberg currents is denoted by $D^{p, q}(U)$. A Lagerberg distribution is a Lagerberg current of type $(n, n)$.

Remark 3.2.9 Let $U$ denote an open subset of $N_{\Sigma}$.

(i) If $U$ is contained in the open generic stratum $N_{\mathbb{R}}$, then the definition of $D^{p, q}(U)$ above coincides with the definition of $D^{p, q}(U)$ given by Lagerberg [16, 2.1].

(ii) By the usual methods, the spaces of Lagerberg currents inherit operators

$$
d^{\prime}: D^{p, q}(U) \longrightarrow D^{p+1, q}(U), d^{\prime \prime}: D^{p, q}(U) \longrightarrow D^{p, q+1}(U)
$$

and a product

$$
A^{p^{\prime}, q^{\prime}}(U) \otimes D^{p, q}(U) \longrightarrow D^{p+p^{\prime}, q+q^{\prime}}(U)
$$


such that

$$
\begin{aligned}
d^{\prime}(T)\left(\omega^{\prime}\right) & =(-1)^{p+q+1} T\left(d^{\prime} \omega^{\prime}\right), \\
d^{\prime \prime}(T)\left(\omega^{\prime \prime}\right) & =(-1)^{p+q+1} T\left(d^{\prime \prime} \omega^{\prime \prime}\right), \\
(\beta \wedge T)(\omega) & =(-1)^{\left(p^{\prime}+q^{\prime}\right)(p+q)} T(\beta \wedge \omega)
\end{aligned}
$$

for all $T \in D^{p, q}(U), \beta \in A^{p^{\prime}, q^{\prime}}(U)$ and all $\omega^{\prime}, \omega^{\prime \prime}, \omega \in A^{\cdot, \cdot}(U)$ of suitable bidegree.

(iii) There is an involution $J$ on $D^{\cdot, \cdot}(U)=\oplus_{p, q \geq 0} D^{p, q}(U)$ such that given $T \in D^{p, q}(U)$ and $\omega \in A_{c}^{n-q, n-p}(U)$ the Lagerberg current $J(T) \in D^{q, p}(U)$ is given by

$$
J(T)(\omega)=(-1)^{n} T(J(\omega)) .
$$

A Lagerberg current $T \in D^{p, p}(U)$ is called symmetric if it satisfies $J(T)=(-1)^{p} T$.

Integration of Lagerberg forms gives examples of currents. We start by recalling the integration theory of Lagerberg forms. If $U \subset N_{\Sigma}$ is an open subset and $\eta \in A^{n, n}(U)$ is a Lagerberg form with compact support, using the chosen basis of $M$, we write

$$
\eta=f d^{\prime} u_{1} \wedge d^{\prime \prime} u_{1} \wedge \cdots \wedge d^{\prime} u_{n} \wedge d^{\prime \prime} u_{n} .
$$

Denote by $d \lambda$ the Lebesgue measure on $N_{\mathbb{R}}$ induced by the lattice $N$. The integral of $\eta$ is defined as

$$
\int_{U} \eta=\int_{N_{\mathbb{R}}} f d \lambda .
$$

Since the support of any compactly supported Lagerberg form of type $(n, n)$ is a compact subset of $N_{\mathbb{R}}$, the integral is finite. Since two isomorphisms $N \cong \mathbb{Z}^{n}$ differ by a matrix of determinant \pm 1 , the integral does not depend on the choice of coordinates.

Example 3.2.10 Let $U \subset N_{\Sigma}$ be an open subset. We will use the map

$$
[]: A^{p, q}(U) \longrightarrow D^{p, q}(U), \quad \eta \longmapsto[\eta](\cdot)=\int_{U} \eta \wedge \cdot
$$

This map is a morphism of $A^{\cdot \cdot}(U)$-modules compatible with the actions of $d^{\prime}, d^{\prime \prime}$ and $J$.

Example 3.2.11 Let $U$ be an open subset of $N_{\Sigma}$. For every real Radon measure $\mu$ on $U$ (see Appendix A), there exists a unique Lagerberg current $T \in D^{n, n}(U)$ such that

$$
T(f)=\int_{U} f d \mu \quad \forall f \in A_{c}^{0,0}(U) .
$$

Indeed, for a compact subset $K$ of $U$ and a finite covering $\left(V_{i}\right)_{i \in I}$ of $K$, the canonical maps $A_{K}^{0,0}\left(U,\left(V_{i}\right)_{i \in I}\right) \rightarrow C_{K}^{0}(U, \mathbb{R})$ and $\left.C_{K}^{0}(U, \mathbb{R}) \rightarrow C_{c}^{0}(U), \mathbb{R}\right)$ are morphisms of locally convex vector spaces. By the universal property of the direct limit, the composition of these maps induces a continuous map $A_{c}^{0,0}(U) \rightarrow C_{c}^{0}(U, \mathbb{R})$. This shows our claim.

Proposition 3.2.12 Let $\left(U_{i}\right)_{i \in I}$ be an open cover of an open subset $U$ of $N_{\Sigma}$. Then there exists a partion of unity subordinate to the given cover $\left(U_{i}\right)_{i \in I}$, i.e. a countable, locally finite open cover $\left(V_{j}\right)_{j \in J}$ of $U$ together with a map $s: J \rightarrow I$ such that $V_{j} \subset U_{s(j)}$ for all $j \in J$ and a collection of non-negative functions $f_{j} \in A_{c}^{0,0}\left(V_{j}\right)$ such that $\sum_{j \in J} f_{j} \equiv 1$. 
Proof By general arguments, see [23, Theorem 1.11], it is sufficient to show that, given a point $x$ and a neighborhood $V$ of $x$ in $U$, there exists a function $f \in A_{c}^{0,0}(V)$ that is constant equal to 1 on a neighborhood of $x$. This statement is clearly local, so we may assume that our fan $\Sigma$ is generated by a single cone $\sigma$ and $N_{\Sigma}=N_{\sigma}$. We have seen in Remark 3.1.2 that a choice of $k$ generators of the cone $\sigma$ leads to a realization of $N_{\sigma}$ as a closed (polyhedral) subset of $\mathbb{R}_{\infty}^{k}$. Now the existence of a partition of unity for an open subset of $\mathbb{R}_{\infty}^{k}$ from [14, Lemma 2.7] readily shows the claim.

Let $U$ be an open subset of $N_{\Sigma}$. Recall from Appendix A that the space $C_{c}^{0}(U, \mathbb{R})$ of real valued continuous functions on $U$ with compact support has a canonical structure of a locally convex topological vector space.

Corollary 3.2.13 For $U \subset N_{\Sigma}$ open, the image of $A_{c}^{0,0}(U) \hookrightarrow C_{c}^{0}(U)$ is sequentially dense.

Proof It is enough to show that, for any continuous function $f \in C_{c}^{0}(U)$, there is a sequence of functions $g_{k} \in A_{c}^{0,0}(U), k \geq 0$, that converges to $f$ in the topology of $C_{c}^{0}(U)$. Let $K$ be the support of $f$. Using that $N_{\Sigma}$ is locally compact, we can easily find open subsets $U_{1}, U_{2}$ and compact subsets $K_{1}, K_{2}$ with

$$
K \subset U_{1} \subset K_{1} \subset U_{2} \subset K_{2} \subset U .
$$

By Proposition 3.2.12, the Stone-Weierstrass Theorem [20, Theorem 7.32] implies that the $\mathbb{R}$-algebra $\left\{\left.h\right|_{K_{1}} \mid h \in A^{0,0}(U), \operatorname{supp}(h) \subset K_{2}\right\}$ is dense in $C^{0}\left(K_{1}\right)$. Hence there is a sequence of smooth functions $h_{k} \in A_{c}^{0,0}(U)$ with $\operatorname{supp}\left(h_{k}\right) \subset K_{2}$ such that the $\left.h_{k}\right|_{K_{1}}$ converge uniformly to $f$ in $C^{0}\left(K_{1}\right)$. Again by Lemma 3.2.12, there is a smooth function $0 \leq \rho \leq 1$, whose support is contained in $U_{1}$ and with $\left.\rho\right|_{K} \equiv 1$. Then the sequence of smooth functions given by $g_{k}=\rho h_{k}$ converges to $f$ in $C_{c}^{0}(U)$.

\section{Positivity for complex invariant forms and Lagerberg forms}

In this section we study positive forms on a smooth complex toric variety that are invariant under the action of the compact torus and compare them to positive Lagerberg forms. We keep the setting from Section 3.

\subsection{Invariant forms in the case of the torus}

We start with the case of the complex algebraic torus $\mathbb{T}=\operatorname{Spec} \mathbb{C}[M]$ of dimension $n$ with character lattice $M$ and cocharacter lattice $N$. We fix a splitting $N \cong \mathbb{Z}^{n}$ that induces holomorphic coordinates $z_{1}, \ldots, z_{n}$ on $T$ as well as linear coordinates $u_{1}, \ldots, u_{n}$ on $N_{\mathbb{R}}$. As before we denote the associated complex manifold $M \otimes_{\mathbb{Z}} \mathbb{C}^{\times} \cong\left(\mathbb{C}^{\times}\right)^{n}$ by $\mathbb{T}^{\text {an }}$. We will also consider the real compact torus

$$
\mathbb{S}:=\left\{z \in \mathbb{T}^{\text {an }}|| z_{j} \mid=1 \quad \forall j \in\{1, \ldots, n\}\right\}=\left\{z \in \mathbb{T}^{\text {an }}|| \chi^{u}(z) \mid=1 \quad \forall u \in M\right\} .
$$

We will denote by $A$ either the sheaf of complex differential forms on $\mathbb{T}^{\text {an }}$ or the sheaf of (real) Lagerberg forms on $N_{\mathbb{R}}$. The context will always allow us to distinguish between them. If not we will denote the former as $A_{\mathbb{T}^{\text {an }}}$ and the latter as $A_{N_{\mathbb{R}}}$. For an $\mathbb{S}$-invariant subset $V$ of $\mathbb{T}^{\text {an }}$, let $A(V)^{\mathbb{S}}$ denote the subalgebra of $A(V)$ given by the $\mathbb{S}$-invariant forms. 
Remark 4.1.1 Let $V$ be an $\mathbb{S}$-invariant subset of $\mathbb{T}^{\text {an }}$. The subalgebra $A(V)^{\mathbb{S}}$ is a direct factor of $A(V)$ because averaging with respect to the Haar probability measure $\mu_{\mathbb{S}}$ of $\mathbb{S}$ defines a canonical projection

$$
A(V) \longrightarrow A(V)^{\mathbb{S}}, \quad \omega \longmapsto \omega^{\mathrm{av}}:=\int_{\mathbb{S}} a^{*}(\omega) d \mu_{\mathbb{S}}(a)
$$

where $a: \mathbb{T}^{\mathrm{an}} \rightarrow \mathbb{T}^{\mathrm{an}}$ denotes translation by $a \in \mathbb{T}^{\mathrm{an}}$.

Definition 4.1.2 Let $F$ be the antilinear involution of the sheaf of $\mathbb{C}$-algebras $A=A_{\mathbb{T}}$ an $\operatorname{deter-}$ mined by $F(f)=\bar{f}$ for $f \in A^{0,0}$ and by

$$
F\left(d z_{j} / z_{j}\right)=d z_{j} / z_{j}, \quad F\left(i d \bar{z}_{j} / \bar{z}_{j}\right)=i d \bar{z}_{j} / \bar{z}_{j}
$$

for $j=1, \ldots, n$. The $\mathbb{S}$-invariant one-forms $d z_{j} / z_{j}$ and $i d \bar{z}_{j} / \bar{z}_{j}(j=1, \ldots, n)$ generate a subsheaf of $\mathbb{R}$-algebras $B$ of $A$ such that $A^{\cdot, \cdot}=A^{0,0} \otimes_{\mathbb{R}} B^{\cdot, \cdot}$. Observe that this definition of $B$ does not depend on the choice of the splitting $N \cong \mathbb{Z}^{n}$ which induces the coordinates $z_{1}, \ldots, z_{n}$. The antilinear involution $F$ above is the $\mathbb{R}$-linear endomorphism on $A_{\mathbb{T}^{\text {an }}}$ given as the tensor product of complex conjugation on $A^{0,0}$ with the identity on $B$. We conclude that the involution $F$ is indeed well defined.

Lemma 4.1.3 The involution $F$ is $\mathbb{T}^{\text {an }}$-equivariant. In particular $F$ induces an antilinear involution, also denoted by $F$, of $A(V)^{\mathbb{S}}$ for any $\mathbb{S}$-invariant open subset $V$ of $\mathbb{T}^{\text {an }}$.

Proof For $f \in A^{0,0}(V)$ and $j=1, \ldots, n$, we have

$$
a^{*} \bar{f}=\overline{a^{*} f}, a^{*}\left(d z_{j} / z_{j}\right)=d z_{j} / z_{j}, \text { and } a^{*}\left(i d \bar{z}_{j} / \bar{z}_{j}\right)=i d \bar{z}_{j} / \bar{z}_{j} .
$$

We deduce that $a^{*} F(\omega)=F\left(a^{*} \omega\right)$ for any $\omega \in A(V)$ and the claim follows.

Lemma 4.1.4 Let $V \subset \mathbb{T}^{\text {an }}$ be an $\mathbb{S}$-invariant open subset. If $\omega \in A(V)^{\mathbb{S}}$, then

$$
F(\partial \omega)=\partial F(\omega), \quad F(i \bar{\partial} \omega)=i \bar{\partial} F(\omega) .
$$

Proof We note that the $\mathbb{S}$-invariant forms $i^{|K|} d z_{I} \wedge d \bar{z}_{K} /\left(z_{I} \bar{z}_{K}\right)$ give a frame in $A(V)$. Using that $r \rightarrow \log \left(r^{2}\right)$ is a diffeomorphism from $\mathbb{R}_{>0}$ onto $\mathbb{R}$, any $\omega \in A(V)^{\mathbb{S}}$ can be written as

$$
\omega=\sum_{I, K} f_{I, K}\left(\log \left(z_{1} \bar{z}_{1}\right), \ldots, \log \left(z_{n} \bar{z}_{n}\right)\right) \frac{i^{|K|} d z_{I} \wedge d \bar{z}_{K}}{z_{I} \bar{z}_{K}}
$$

where the functions $f_{I, K}$ are smooth complex valued functions of $n$ real variables. We denote by $\partial_{j} f_{I, K}$ the partial derivative with respect to the $j$-th variable. Clearly

$$
\partial_{j} \bar{f}_{I, K}=\overline{\partial_{j} f_{I, K}} \text {. }
$$

Then $F(\partial \omega)$ is equal to

$$
F\left(\sum_{I, K} \sum_{j \notin I} \partial_{j} f_{I, K} \frac{d z_{j}}{z_{j}} \wedge \frac{i^{|K|} d z_{I} \wedge d \bar{z}_{K}}{z_{I} \bar{z}_{K}}\right)=\sum_{I, K} \sum_{j \notin I} \overline{\partial_{j} f_{I, K}} \frac{d z_{j}}{z_{j}} \wedge \frac{i^{|K|} d z_{I} \wedge d \bar{z}_{K}}{z_{I} \bar{z}_{K}}
$$

and

$$
\partial F(\omega)=\partial\left(\sum_{I, K} \bar{f}_{I, K} \frac{i^{|K|} d z_{I} \wedge d \bar{z}_{K}}{z_{I} \bar{z}_{K}}\right)=\sum_{I, K} \sum_{j \notin I} \partial_{j} \bar{f}_{I, K} \frac{d z_{j}}{z_{j}} \wedge \frac{i^{|K|} d z_{I} \wedge d \bar{z}_{K}}{z_{I} \bar{z}_{K}} .
$$


Thus the commutativity between $\partial$ and $F$ follows from $\partial_{j} \bar{f}_{I, K}=\overline{\partial_{j} f_{I, K}}$.

Similarly, $F(i \bar{\partial} \omega)$ is equal to

$$
F\left(\sum_{I, K} \sum_{j \notin K} \partial_{j} f_{I, K} \frac{i d \bar{z}_{j}}{\bar{z}_{j}} \wedge \frac{i^{|K|} d z_{I} \wedge d \bar{z}_{K}}{z_{I} \bar{z}_{K}}\right)=\sum_{I, K} \sum_{j \notin K} \frac{\bar{\partial}_{j} f_{I, K}}{i d \bar{z}_{j}} \bar{z}_{j} \wedge \frac{i^{|K|} d z_{I} \wedge d \bar{z}_{K}}{z_{I} \bar{z}_{K}}
$$

and

$$
i \bar{\partial} F(\omega)=i \bar{\partial}\left(\sum_{I, K} \bar{f}_{I, K} \frac{i^{|K|} d z_{I} \wedge d \bar{z}_{K}}{z_{I} \bar{z}_{K}}\right)=\sum_{I, K} \sum_{j \notin K} \partial_{j} \bar{f}_{I, K} \frac{i d \bar{z}_{j}}{\bar{z}_{j}} \wedge \frac{i^{|K|} d z_{I} \wedge d \bar{z}_{K}}{z_{I} \bar{z}_{K}} .
$$

Therefore the commutativity of $F$ and $i \bar{\partial}$ also follows from $\partial_{j} \bar{f}_{I, K}=\overline{\partial_{j} f_{I, K}}$.

The next example shows that $\mathbb{S}$-invariance of $\omega$ is necessary in Lemma 4.1.4.

Example 4.1.5 Consider the case $n=1$ and let $f(z)=z+\bar{z}$. This function is real but not S-invariant. Furthermore

$$
\partial F(f)=\partial f=d z
$$

and

$$
F(\partial f)=F(d z)=F(z d z / z)=\bar{z} d z / z
$$

Definition 4.1.6 For any $\mathbb{S}$-invariant open subset $V \subset \mathbb{T}^{\text {an }}$, we denote by $A(V)^{\mathbb{S}, F}$ the algebra of forms that are simultaneously $F$-invariant and $\mathbb{S}$-invariant. Since $F$ and the action of $\mathbb{S}$ both respect the bigrading of $A$, we deduce that $A(V)^{\mathbb{S}, F}$ is a bigraded algebra. By Lemma 4.1.4, the operators $\partial$ and $i \bar{\partial}$ induce operators on $A(V)^{\mathbb{S}, F}$.

The next goal is to give an identification between the algebra of $\mathbb{S}$-invariant forms that are also $F$-invariant, and the algebra of real Lagerberg forms on $N_{\mathbb{R}}$. This identification will respect the natural differential operators. Recall that $J$ denotes the Lagerberg involution introduced in Remark 3.2.2.

Proposition 4.1.7 Let $U \subset N_{\mathbb{R}}$ be an open subset and $V=\operatorname{trop}^{-1}(U)$ the corresponding $\mathbb{S}$-invariant subset of $\mathbb{T}^{\text {an }}$. Then there is a unique homomorphism

$$
\text { trop }^{*}: A(U) \longrightarrow A(V)
$$

of $\mathbb{R}$-algebras such that

$$
\operatorname{trop}^{*}(\varphi)=\varphi \circ \text { trop }
$$

for all $\varphi \in A^{0,0}(U)$ and such that

$$
\operatorname{trop}^{*}\left(d^{\prime} \omega\right)=\pi^{-1 / 2} \partial \operatorname{trop}^{*}(\omega) \text { and } \operatorname{trop}^{*}\left(d^{\prime \prime} \omega\right)=\pi^{-1 / 2} i \bar{\partial} \operatorname{trop}^{*}(\omega)
$$

for all $\omega \in A(U)$. Moreover, this homomorphism is injective with image $A(V)^{\mathbb{S}, F}$ inducing an isomorphism $A(U) \simeq A(V)^{\mathbb{S}, F}$. For $\omega \in A^{p, q}(U)$, we have

$$
\operatorname{trop}^{*}(J(\omega))=i^{p+q} \overline{\operatorname{trop}^{*}(\omega)} .
$$


Proof We first recall some formulas from complex analysis in one variable. If $z=r e^{i \theta}$ and $u=-\log |z|=-\log (r)$, since $r^{2}=z \bar{z}$, we have

$$
\partial(u)=-\frac{d z}{2 z} \text { and } \bar{\partial} u=-\frac{d \bar{z}}{2 \bar{z}} .
$$

For $j=1, \ldots, n$ write $r_{j}=\left|z_{j}\right|=\left(z_{j} \bar{z}_{j}\right)^{1 / 2}$. Then

$$
\operatorname{trop}^{*}\left(u_{j}\right)=-\log \left(r_{j}\right) \text {. }
$$

Therefore, Eqs. (4.2) and (4.3) imply

$$
\operatorname{trop}^{*}\left(d^{\prime} u_{j}\right)=-\frac{d z_{j}}{2 \sqrt{\pi} z_{j}}, \quad \operatorname{trop}^{*}\left(d^{\prime \prime} u_{j}\right)=-\frac{i d \bar{z}_{j}}{2 \sqrt{\pi} \bar{z}_{j}} .
$$

Since trop* is an algebra homomorphism, we deduce that, if $\omega \in A(U)$ is written as

$$
\omega=\sum_{I, K} f_{I, K}\left(u_{1}, \ldots, u_{n}\right) d^{\prime} u_{I} \wedge d^{\prime \prime} u_{K},
$$

then the corresponding form on the torus is given by

$$
\operatorname{trop}^{*}(\omega)=\sum_{I, K}\left(\frac{-1}{2 \sqrt{\pi}}\right)^{|I|+|K|} i^{|K|} f_{I, K}\left(-\log \left(r_{1}\right), \ldots,-\log \left(r_{n}\right)\right) \frac{d z_{I} \wedge d \bar{z}_{K}}{z_{I} \wedge \bar{z}_{K}} .
$$

This proves the uniqueness of the map trop*. Conversely, using Eq. (4.7) as the definition of trop*, the uniqueness of the decomposition (4.6) shows that trop* is well defined and it is immediate to verify (4.2), (4.3) and (4.4). Clearly, the form in (4.7) is $F$ - and $\mathbb{S}$-invariant and every element in $A(V)^{\mathbb{S}, F}$ has this form. Obviously, the map trop* is injective. Hence it induces an isomorphism $A(U) \simeq A(V)^{\mathbb{S}, F}$.

Corollary 4.1.8 Let $U \subset N_{\mathbb{R}}$ be an open subset and $V=\operatorname{trop}^{-1}(U)$. Then trop* induces an isomorphism of topological vector spaces $A_{c}(U) \simeq A_{c}(V)^{\mathbb{S}, F}$.

Proof The isomorphism of vector spaces follows from Proposition 4.1.7 as the map trop is proper. Using that trop is a submersion, the topological statement can be checked locally in coordinates.

Remark 4.1.9 The $\mathbb{S}$-invariant open subsets of $\mathbb{T}^{\text {an }}$ are precisely the preimages of open subsets of $N_{\mathbb{R}}$. Hence $\mathbb{S}$ and $F$ act in a natural way on the sheaf $\operatorname{trop}_{*} A$. We denote by $\left(\operatorname{trop}_{*} A\right)^{\mathbb{S}, F}$ the subsheaf of sections invariant under $\mathbb{S}$ and $F$. Then Proposition 4.1.7 yields a monomorphism of sheaves of $\mathbb{R}$-algebras

$$
\operatorname{trop}^{*}: A \longrightarrow\left(\operatorname{trop}_{*} A\right)^{\mathbb{S}, F}
$$

that preserves differentials as in (4.3).

The next remark shows that Lagerberg forms and the involution $F$ are pointwise described by the linear algebra in Sect. 2 .

Remark 4.1.10 Choose a point $x \in \mathbb{T}^{\text {an }}$ and let $y=-\log |x|=\operatorname{trop}(x)$ denote its image under the tropicalization map. Write $V=T_{y} N_{\mathbb{R}}$ for the tangent space to $N_{\mathbb{R}}$ at $y$. Let $V^{\prime}$, $V^{\prime \prime}, V^{*}$ and $\overline{V^{*}}$ be defined as Sect. 2. There is an isomorphism

$$
\operatorname{trop}_{x}^{*}: V^{*} \oplus \overline{V^{*}} \longrightarrow T_{x}^{1,0} \mathbb{T}^{\text {an }} \oplus T_{x}^{0,1} \mathbb{T}^{\text {an }}
$$


given by

$$
d u_{j} \longmapsto-\frac{d z_{j}}{2 \sqrt{\pi} x_{j}}, \quad \text { and } i d \bar{u}_{j} \longmapsto \frac{-i d \bar{z}_{j}}{2 \sqrt{\pi} \bar{x}_{j}} .
$$

This isomorphism is compatible with the map trop* in the sense that

$$
\operatorname{trop}^{*}(\omega)(x)=\operatorname{trop}_{x}^{*}(\omega(y)) .
$$

Let $\tau_{n} \in \Lambda^{n, n} V^{\prime}$ be the Lagerberg orientation as in Definition 2.2.1. Then

$$
\operatorname{trop}_{x}^{*}\left(\tau_{n}\right)=\frac{i^{n} d z_{1} \wedge d \bar{z}_{1} \wedge \cdots \wedge d z_{n} \wedge d \bar{z}_{n}}{(4 \pi)^{n}\left|x_{1}\right|^{2} \ldots\left|x_{n}\right|^{2}} .
$$

Note that the denominator of this form is strictly positive in the torus $\mathbb{T}^{\text {an }}$ therefore, any positivity notions on $\mathbb{T}^{\text {an }}$ defined using the orientation $\operatorname{trop}_{x}^{*}\left(\tau_{n}\right)$ or the orientation

$$
i^{n} d z_{1} \wedge d \bar{z}_{1} \wedge \cdots \wedge d z_{n} \wedge d \bar{z}_{n}
$$

agree.

Let $\omega \in A^{p, q}(W)$ for some open subset $W$ of $\mathbb{T}^{\text {an }}$ and $x \in W$. We get from (2.5) that

$$
F(\omega)(x)=F(\omega(x))
$$

where $F$ on the righthand side is the involution from Definition 2.3.1.

Definition 4.1.11 Let $U$ be an open set of $N_{\mathbb{R}}$. A Lagerberg form $\omega \in A(U)$ is called strongly positive (respectively positive, weakly positive) if for all $x \in U, \omega(x)$ is strongly positive (respectively positive, weakly positive).

Let $V$ be an open set of $\mathbb{T}^{\text {an }}$. A complex differential form $\omega \in A(V)$ is called strongly positive (respectively positive, weakly positive) if for all $y \in V, \omega(y)$ is strongly positive (respectively positive, weakly positive).

As we have seen in Example 2.3.6, it is reasonable to restrict our attention to positive Lagerberg forms. Nevertheless we add the other positivity notions for further reference.

Lemma 4.1.12 Let $U$ be an open set of $N_{\mathbb{R}}$ and $\omega \in A^{p, p}(U)$ be a Lagerberg form on $U$.

(i) The Lagerberg form $\omega$ is symmetric if and only if trop* $\omega$ is real.

(ii) If $\omega$ is strongly positive, then trop* $\omega$ is strongly positive.

(iii) If trop* $\omega$ is weakly positive then $\omega$ is weakly positive.

(iv) The Lagerberg form $\omega$ is positive if and only if trop* $\omega$ is positive.

Proof The Lagerberg form $\omega$ is symmetric if and only if $J \omega=(-1)^{p} \omega$. By Eq. (4.4) this is equivalent to trop* $(\omega)=\overline{\operatorname{trop}^{*}(\omega)}$. This proves the first statement.

The remaining assertions follow from the fact that positivity is checked pointwise, Remark 4.1.10 and Propositions 2.3.4 and 2.3.5.

Remark 4.1.13 Remark 4.1.10 shows that the correspondence

$$
\pi^{-1 / 2} \partial \longleftrightarrow d^{\prime} \text { and } \pi^{-1 / 2} i \bar{\partial} \longleftrightarrow d^{\prime \prime}
$$

from Proposition 4.1.7 was already used implicitly in Section 2 in order to preserve positivity and the bigrading between the complex and the Lagerberg forms. 
Remark 4.1.14 Chambert-Loir and Ducros mention in [7, Remarque 1.2.12] the identifications $d=\partial+\bar{\partial} \leftrightarrow d^{\prime}$ and $4 \pi d^{c}=\frac{1}{i}(\partial-\bar{\partial}) \leftrightarrow d^{\prime \prime}$ (observe that $\left.d \operatorname{Arg}(z)=4 \pi d^{c} \log |z|\right)$ which have the disadvantage that they do not respect the bigrading and do not allow the nice interpretation of symmetric Lagerberg forms as real complex forms as in Lemma 4.1.12.

Remark 4.1.15 Let $U$ be an open subset of $N_{\mathbb{R}}$ and $V:=\operatorname{trop}^{-1}(U)$. We have introduced the map trop*: $A(U) \rightarrow A(V)$ in order to establish later correspondence results between Lagerberg forms and currents on $U$ and invariant complex forms and currents on $V$.

Let us explain our choices which lead to the achieved correspondence (4.11): Our first condition is that trop* should be a differential homomorphism of $\mathbb{R}$-algebras with respect to the differential operators $d^{\prime}$ and $d^{\prime \prime}$ of $A(U)$ and natural differential operators $\partial^{\prime}$ and $\partial^{\prime \prime}$ on $A(V)$. Naturality means here that $\partial^{\prime}, \partial^{\prime \prime}$ should be in the two-dimensional $\mathbb{C}$-vector space spanned by $\partial$ and $\bar{\partial}$. The second condition is that trop* respects the bigrading which implies $\partial^{\prime}=a \partial$ and $\partial^{\prime \prime}=b i \bar{\partial}$ for some $a, b \in \mathbb{C}$. Third, the range of trop* should be contained in the $F$-invariant forms in $V$ which yields $a, b \in \mathbb{R}$. Observe that these three conditions imply already that trop* preserves positivity of forms (see Lemma 4.1.12). Our fourth condition is (4.4) which forces $a=b$. The fifth condition is that trop* is compatible with integration (see Lemma 4.2 .5 below). This gives $a b=1 / \pi$. Hence we have seen that the five conditions given above fix our choices in Proposition 4.1.7 up to a sign.

\subsection{Invariant forms in the case of a toric variety}

The next goal is to partially extend Proposition 4.1.7 to toric varieties. Let $\Sigma$ be a smooth fan in $N_{\mathbb{R}}$. Let $X_{\Sigma}$ be the corresponding smooth complex toric variety and let $N_{\Sigma}$ be the corresponding partial compactification of $N_{\mathbb{R}}$ as in Sect. 3. We denote by $X_{\Sigma}^{\text {an }}$ the complex manifold associated to $X_{\Sigma}$. Recall from Remark 3.1.3 that the tropicalization map is a proper continuous map trop: $X_{\Sigma}^{\text {an }} \rightarrow N_{\Sigma}$ that identifies $N_{\Sigma}$ with $X_{\Sigma}^{\text {an }} / \mathbb{S}$.

Remark 4.2.1 Let $V \subset X_{\Sigma}^{\text {an }}$ be an $\mathbb{S}$-invariant open subset. Then, in general, the involution $F$ does not induce an involution of $A^{\cdot, \cdot}(V)$. In fact, if $\omega \in A^{\cdot, \cdot}(V)$, then $F\left(\left.\omega\right|_{V \cap \mathbb{T}^{\text {an }}}\right)$ may not extend to a smooth form on $V$, as the following example shows.

We set $X_{\Sigma}=\mathbb{A}_{\mathbb{C}}^{1}$ and $V=X_{\Sigma}^{\text {an }}=\mathbb{C}$. Then $d z \in A^{1,0}(V)$, but

$$
F(d z)=F\left(z \frac{d z}{z}\right)=\frac{\bar{z}}{z} d z
$$

is not a smooth form in 0 . Nevertheless, the next result implies that $F$ can be extended to smooth $\mathbb{S}$-invariant forms.

Lemma 4.2.2 Let $V \subset X_{\Sigma}^{\mathrm{an}}$ be an $\mathbb{S}$-invariant open subset. Let $\omega \in A^{\cdot \cdot \cdot}(V)^{\mathbb{S}}$ be a smooth

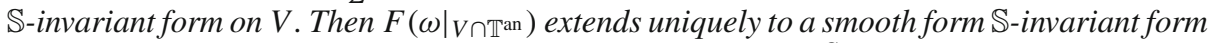
on $V$ and hence $F$ induces an antilinear involution on $A^{\cdot, \cdot}(V)^{\mathbb{S}}$ also denoted by $F$.

Proof Since the statement is local we may assume that $V$ is contained in the affine toric variety $X_{\sigma}$ for some $\sigma \in \Sigma$. We choose a system of toric coordinates $\left(z_{1}, \ldots, z_{n}\right)$ and write

$$
\omega=\sum f_{I, K}\left(z_{1}, \ldots, z_{n}\right) d z_{I} \wedge d \bar{z}_{K} .
$$

Moreover, the $\mathbb{S}$-invariance of $V$ yields that $V$ is invariant under complex conjugation of the coordinates. Since $\omega$ is $\mathbb{S}$-invariant, each summand $f_{I, K} d z_{I} \wedge d \bar{z}_{K}$ is also $\mathbb{S}$-invariant and 
hence we can write

$$
f_{I, K}\left(z_{1}, \ldots, z_{n}\right) d z_{I} \wedge d \bar{z}_{K}=g_{I, K}\left(\left|z_{1}\right|, \ldots,\left|z_{n}\right|\right) \frac{i^{|J|} d z_{I} \wedge d \bar{z}_{K}}{z_{I} \bar{z}_{K}},
$$

for a unique smooth function $g_{I, K} \in C^{\infty}(V)$. On $V \cap \mathbb{T}^{\text {an }}$, this yields

$$
\begin{aligned}
F\left(f_{I, K}\left(z_{1}, \ldots, z_{n}\right) d z_{I} \wedge d \bar{z}_{K}\right) & =F\left(g_{I, K}\left(\left|z_{1}\right|, \ldots,\left|z_{n}\right|\right) \frac{i^{|K|} d z_{I} \wedge d \bar{z}_{K}}{z_{I} \bar{z}_{K}}\right) \\
& =\overline{g_{I, K}\left(\left|z_{1}\right|, \ldots,\left|z_{n}\right|\right)} \frac{i^{|K|} d z_{I} \wedge d \bar{z}_{K}}{z_{I} \bar{z}_{K}} \\
& =(-1)^{|K|} \overline{f_{I, K}\left(\bar{z}_{1}, \ldots, \bar{z}_{n}\right)} d z_{I} \wedge d \bar{z}_{K}
\end{aligned}
$$

which can be uniquely extended to a smooth form on $V$ because $\omega$ is smooth. By Lemma 4.1.3, the restriction $\left.F(\omega)\right|_{V \cap \mathbb{T}}$ in $\mathbb{S}$-invariant. By continuity, $F(\omega)$ is $\mathbb{S}$-invariant.

For any open $\mathbb{S}$-invariant subset $V \subset X_{\Sigma}^{\text {an }}$, we will denote by $A(V)^{\mathbb{S}, F}$ the $\mathbb{R}$-subalgebra of forms that are at the same time invariant under $\mathbb{S}$ and under $F$. As before we obtain a sheaf of bigraded $\mathbb{R}$-algebras $\left(\operatorname{trop}_{*} A_{X_{\Sigma}}\right)^{\mathbb{S}, F}$ on $N_{\Sigma}$.

Proposition 4.2.3 There is a unique morphism of sheaves of bigraded $\mathbb{R}$-algebras

$$
\text { trop* }: A_{N_{\Sigma}} \longrightarrow\left(\operatorname{trop}_{*} A_{X_{\Sigma}^{\text {an }}}\right)^{\mathbb{S}, F}
$$

that extends the morphism (4.8). Moreover, trop* satisfies

$$
\pi^{-1 / 2} \partial \circ \text { trop}^{*}=\text { trop}^{*} \circ d^{\prime} \text { and } \pi^{-1 / 2} i \bar{\partial} \circ \text { trop }^{*}=\text { trop* }^{*} \circ d^{\prime \prime} .
$$

For an open subset $U$ of $N_{\Sigma}$ and $\omega \in A^{p, q}(U)$, we have

$$
\operatorname{trop}^{*}(J(\omega))=i^{p+q} \overline{\operatorname{trop}^{*}(\omega)} .
$$

Proof This follows easily from the definitions and Proposition 4.1.7.

Remark 4.2.4 If $U$ is an open subset of $N_{\mathbb{R}}$, then we have seen in Proposition 4.1.7 that the map (4.12) is an isomorphism. For an open subset $U$ of $N_{\Sigma}$, the map is obviously still injective, but in general no longer surjective. The latter can be seen already in the one dimensional case. Let $N=\mathbb{Z}$ and $\Sigma$ the fan with a single maximal cone $\sigma=\mathbb{R}_{\geq 0}$. Then $X_{\Sigma}=\mathbb{A}_{\mathbb{C}}^{1}$ and $N_{\Sigma}=\mathbb{R} \cup\{\infty\}$. Consider the smooth function $\varphi(z)=z \bar{z}$ on $X_{\Sigma}^{\text {an }}=\mathbb{C}$ and the function $f(u)=e^{-2 u}$ on $N_{\mathbb{R}}=\mathbb{R}$. Then, on $\mathbb{C}^{\times} \subset \mathbb{C}$ we have $\varphi=\operatorname{trop}^{*} f$, but $f$ can not be extended to a smooth function on $N_{\Sigma}$, because, by definition, a smooth function on $N_{\Sigma}$ has to be constant in a neighborhood of $\infty$. This gives an example of a non-surjective

$$
\text { trop* }: A^{0,0}(U) \longrightarrow A^{0,0}\left(\operatorname{trop}^{-1}(U)\right)^{\mathbb{S}, F} \text {. }
$$

Lemma 4.2.5 Let $U$ be an open set of $N_{\Sigma}$ and $V=\operatorname{trop}^{-1}(U)$. Let $\eta$ be a Lagerberg $(n, n)$-form with compact support contained in $U$. Then

$$
\int_{U} \eta=\int_{V} \operatorname{trop}^{*} \eta
$$

Proof Since $\eta$ is a top degree Lagerberg form with compact support in $U$, it has compact support contained in $U \cap N_{\mathbb{R}}$. Choosing integral linear coordinates in $N_{\mathbb{R}}$, we have

$$
\eta=f\left(u_{1}, \ldots, u_{n}\right) d^{\prime} u_{1} \wedge d^{\prime \prime} u_{1} \wedge \cdots \wedge d^{\prime} u_{n} \wedge d^{\prime \prime} u_{n}
$$


for a smooth function $f$ on $\mathbb{R}^{n}$. By Proposition 4.1.7, we have

$$
\operatorname{trop}^{*} \eta=f\left(-\log \left|z_{1}\right|, \ldots,-\log \left|z_{n}\right|\right) \frac{i^{n} d z_{1} \wedge d \bar{z}_{1} \wedge \cdots \wedge d z_{n} \wedge d \bar{z}_{n}}{(4 \pi)^{n} z_{1} \ldots z_{n} \bar{z}_{1} \ldots \bar{z}_{n}}
$$

Writing trop* $\eta$ in polar coordinates $z_{j}=r_{j} e^{i \theta_{j}}$ and using that

$$
\frac{i d z_{j} \wedge d \bar{z}_{j}}{2 z_{j} \bar{z}_{j}}=\frac{d r_{j} \wedge d \theta_{j}}{r_{j}},
$$

we deduce

$$
\begin{aligned}
\int_{V} \operatorname{trop}^{*} \eta & =\int_{V} f\left(-\log r_{1}, \ldots,-\log r_{n}\right) \frac{d r_{1} \ldots d r_{n} d \theta_{1} \ldots d \theta_{n}}{(2 \pi)^{n} r_{1} \ldots r_{n}} \\
& =\int_{U} f\left(x_{1}, \ldots, x_{n}\right) d x_{1} \ldots d x_{n}=\int_{U} \eta
\end{aligned}
$$

which proves the claim.

4.2.6. We next discuss the notions of positivity for toric varieties. In the case of the complex smooth toric variety $X_{\Sigma}^{\text {an }}$ the different notions of positivity are the usual ones: A complex differential form is strongly positive (resp. positive, weakly positive) if it is so pointwise.

In the case of $N_{\Sigma}$ a little bit more has to be said because the different fibers of the sheaves of forms $A$ over a point $p \in N_{\Sigma} \backslash N_{\mathbb{R}}$ have a different nature.

Recall from Sect. 3.1 that given a point $v \in N_{\Sigma}$, there is an unique orbit $N(\sigma)$ with $v \in N(\sigma)$ corresponding to a cone $\sigma$ of the fan. Then there is an identification of fibers

$$
A_{N_{\Sigma}}(v)=A_{N(\sigma)}(v) .
$$

Therefore, the different notions of positivity for Lagerberg forms make sense for this fiber by using Definition 2.2.1 for $V=N(\sigma)$. Then for $U \subset N_{\Sigma}$, a Lagerberg form is strongly positive (resp. positive, weakly positive) if it is so fiber by fiber.

Lemma 4.2.7 Let $U \subset N_{\Sigma}$ (respectively $V \subset X_{\Sigma}^{\mathrm{an}}$ ) be an open subset and $\omega \in A(U)$ (respectively $\omega \in A(V)$ ) then $\omega$ is strongly positive, positive or weakly positive if and only if the same if true for $\left.\omega\right|_{U \cap N_{\mathbb{R}}}$ (respectively $\left.\omega\right|_{V \cap \mathbb{T}^{\text {an }}}$ ).

Proof This follows from a continuity argument using that the cones of strongly positive, positive and weakly positive Lagerberg forms in each fiber are closed by Corollary 2.2.5.

Lemma 4.2.8 Let $U$ be an open subset of $N_{\Sigma}$ and $V=\operatorname{trop}^{-1}(U) \subset X_{\Sigma}^{\mathrm{an}}$. Let $\eta \in A^{p, p}(U)$.

(i) If $\eta$ is strongly positive, then trop* $(\eta)$ is strongly positive.

(ii) $\eta$ is positive if and only if $\operatorname{trop}^{*}(\eta)$ is positive.

(iii) If trop* $(\eta)$ is weakly positive, then $\eta$ is weakly positive.

Proof The result follows from Lemmas 4.2.7 and 4.1.12.

One can deduce from Example 2.3.8 that the converses of (i) and (iii) in the previous lemma are not always true.

Lemma 4.2.9 Let $U$ be an open subset of $N_{\Sigma}$ and $V=\operatorname{trop}^{-1}(U) \subset X_{\Sigma}^{\mathrm{an}}$. Every strongly positive complex differential form on $V$ (resp. strongly positive Lagerberg form on $U$ ) is positive and every positive complex differential form on $V$ (resp. positive Lagerberg form on $U$ ) is weakly positive. Moreover, if $p=0,1, n-1, n$, then all three positivity notions agree on $A^{p, p}(U)$ (resp. on $\left.A^{p, p}(V)\right)$. 
Proof Since all notions of positivity of forms are checked fiber by fiber, the result follows from Corollary 2.1.6 and Remark 2.1.7 in the complex case and Corollary 2.2.5 and Remark 2.2.6 in the Lagerberg case.

\section{Positivity for complex invariant currents and Lagerberg currents}

Throughout this section, $\Sigma$ will be a smooth fan in $N_{\mathbb{R}}, X_{\Sigma}$ will denote the associated toric variety, $N_{\Sigma}$ will denote the partial compactification of $N_{\mathbb{R}}$ and $X_{\Sigma}^{\text {an }}=X_{\Sigma}(\mathbb{C})$ will denote the complex manifold associated to $X_{\Sigma}$, with tropicalization map trop: $X_{\Sigma}^{\mathrm{an}} \rightarrow N_{\Sigma}$.

\subsection{Invariant currents}

Let $U$ be an open subset of $N_{\Sigma}$ and write $V=\operatorname{trop}^{-1}(U)$. Let $A_{c}^{p . q}(U)$ be the space of Lagerberg forms on $U$ with compact support and similarly $A_{c}^{p, q}(V)$ is the space of complex forms on $V$ with compact support. Since trop: $V \rightarrow U$ is proper, the map trop* in (4.12) induces a map

$$
\operatorname{trop}^{*}: A_{c}^{p, q}(U) \longrightarrow A_{c}^{p, q}(V) .
$$

We now compare the topologies on the space of Lagerberg forms and the space of complex forms through the map trop*. The definition of the $C^{\infty}$-topology on both spaces is slightly different. The topology of $A_{c}^{p, q}(U)$ has been described in Definition 3.2.4. The topology of $A_{c}^{p, q}(V)$ is defined similarly. One first defines a topology on $A_{K}^{p, q}(V)$ for each compact $K$ using norms similar to those in (3.4) taking into account all complex derivatives and then define the topology of $A_{c}^{p, q}(V)$ as the direct limit in the category of locally convex topological vector spaces. So the main difference is the use of finite coverings in Definition 3.2.4. From the definition of the topologies, it is easy to check that the map trop* $: A_{c}^{p, q}(U) \rightarrow A_{c}^{p, q}(V)$ is injective and continuous. Nevertheless, as the following examples show, trop* is in general not a homeomorphism onto its image endowed with the subspace topology. Moreover, the image of trop* is not closed in $A_{c}^{p, q}(V)$.

Example 5.1.1 As in Remark 4.2.4, we consider the case $X_{\Sigma}=\mathbb{A}_{\mathbb{C}}^{1}$, thus $N_{\Sigma}=\mathbb{R}_{\infty}$. Consider $U=\left\{u \in \mathbb{R}_{\infty} \mid u>0\right\} \subset N_{\Sigma}$ and $V=\operatorname{trop}^{-1}(U)=\{z \in \mathbb{C} \mid z \bar{z}<1\}$. Let $\rho: \mathbb{R} \rightarrow \mathbb{R}$ be a smooth function with $0 \leq \rho \leq 1$ and $\rho(x)=0$ for $|x|>2$ and $\rho(x)=1$ for $|x|<1$. Define the sequence of functions

$$
f_{n}(u)=\frac{1}{e^{n^{2}}} \rho(u-n) .
$$

The sequence $\left(f_{n}\right)_{n \geq 3}$ does not converge to zero in $A_{c}^{0,0}(U)$ because for any compact subset $K$ of $U$ there is no finite covering $\left\{V_{i}\right\}_{i}$ of $K$ with $f_{n} \in A_{K}^{0,0}\left(U,\left\{V_{i}\right\}\right)$ for all $n$ (see Proposition 3.2.6). Indeed, assume that such a compact $K$ and covering $\left\{V_{i}\right\}$ exists. Since the point $n \in \mathbb{R}_{\infty}$ is in the support of $f_{n}$, and these points converge to $\infty$, then $\infty \in K$. Therefore one $V_{i}$ would contain $\infty$ and hence by definition of $A_{K}^{0,0}\left(U,\left\{V_{i}\right\}\right)$ all the $\left.f_{n}\right|_{V_{i}}$ would have to be constant, which is not the case.

Write $\varphi_{n}=$ trop $^{*} f_{n}$. Then

$$
\varphi_{n}(z)=\frac{1}{e^{n^{2}}} \rho(-\log |z|-n) .
$$


The support of $\varphi_{n}$ is contained in the closed annulus $\left\{\left.z \in \mathbb{C}\left|e^{n-2} \leq\right| z\right|^{-1} \leq e^{n+2}\right\}$. Since $\rho$ is smooth with compact support, all the derivatives of $\rho$ are bounded. From this and the condition above on the support of $\varphi_{n}$, we deduce that for every pair of integers $a, b$, there is a constant $C_{a, b}$ such that

$$
\left|\left(\frac{\partial}{\partial z}\right)^{a}\left(\frac{\partial}{\partial \bar{z}}\right)^{b} \varphi_{n}\right| \leq \frac{1}{e^{n^{2}}} C_{a, b} \sup _{z \in \operatorname{supp}\left(\varphi_{n}\right)} \frac{1}{|z|^{a+b}} \leq \frac{1}{e^{n^{2}}} C_{a, b} e^{(a+b)(n+2)} .
$$

It follows that the sequence $\left(\varphi_{n}\right)_{n \geq 3}$ converges to the function 0 in $A_{c}^{0,0}(V)$. We conclude that the topology of $A_{c}^{0,0}(U)$ is not induced by the topology of $A_{c}^{0,0}(V)$.

Example 5.1.2 We keep the setting from Example 5.1.1. Choose now $\rho \in A^{0,0}(\mathbb{R})$ with $0 \leq \rho \leq 1$ and $\rho(x)=1$ for $x \leq 0$ and $\rho(x)=0$ for $x>1$. Further pick $\psi \in A_{c}^{0,0}(U)$ with $\psi(x)=1$ for $x \geq 1$. Consider the sequence of differential forms

$$
\eta_{n}=\psi(-\log |z|) e^{-1 /|z|} \rho(-\log |z|-n) \frac{i d z \wedge d \bar{z}}{4 \pi z \bar{z}} \in A_{c}^{1,1}(V) \quad(n \in \mathbb{N})
$$

and the differential form $\eta=\psi(-\log |z|) e^{-1 /|z|} i d z \wedge d \bar{z} /(4 \pi z \bar{z})$. Similarly as in Example 5.1.1 the forms $\eta_{n}$ converge to $\eta$ in $A_{c}^{1,1}(V)$. The forms $\eta_{n}$ are in the image of trop* as

$$
\eta_{n}=\operatorname{trop}^{*}\left(\psi(u) \exp \left(-e^{u}\right) \rho(u-n) d^{\prime} u \wedge d^{\prime \prime} u\right),
$$

but the form $\eta$ is not in the image of trop* because the function $\psi(u) e^{-e^{u}}$ is non-constant close to $\infty$. Hence the image of trop* $A_{c}^{1,1}(U) \rightarrow A_{c}^{1,1}(V)$ is not closed.

Now we shift our attention to currents.

Remark 5.1.3 Let $V \subset X_{\Sigma}^{\text {an }}$ be an $\mathbb{S}$-invariant open subset. Similarly as in Remark 4.1.1, the average with respect to the probability Haar measure on $\mathbb{S}$ leads to a canonical projection $A_{c}(V) \rightarrow A_{c}(V)^{\mathbb{S}}$ that we denote $\omega \mapsto \omega^{\text {av }}$. A current $S$ is called $\mathbb{S}$-invariant if

$$
a_{*} S=S \quad \forall a \in \mathbb{S} .
$$

The space of $\mathbb{S}$-invariant currents is denoted $D(V)^{\mathbb{S}}=\bigoplus D \cdot \cdot(V)^{\mathbb{S}}$. There is a canonical projection $D(V) \rightarrow D(V)^{\mathbb{S}}$, where $S$ is mapped to the $\mathbb{S}$-invariant current $S^{\text {av }}$ given by $S^{\mathrm{av}}(\omega)=S\left(\omega^{\mathrm{av}}\right)$. The antilinear involution $F$ of $A(V)^{\mathbb{S}}$ leaves $A_{c}(V)^{\mathbb{S}}$ invariant. It defines an antilinear involution on the space of $\mathbb{S}$-invariant complex currents $D(V)^{\mathbb{S}}$, which we also denote by $F$, given by

$$
(F S)(\eta)=\overline{S(F \eta)} \quad\left(\eta \in A_{c}(V)^{\mathbb{S}}\right) .
$$

The space of $\mathbb{S}$ - and $F$-invariant currents is denoted by $D(V)^{\mathbb{S}, F}$. Observe that $F$ respects the grading. Hence the spaces $D^{p, q}(V)^{\mathbb{S}, F}$ are well-defined. Since $F$ is an involution, there is a canonical projection $D(V)^{\mathbb{S}} \rightarrow D(V)^{\mathbb{S}, F}$.

Definition 5.1.4 The direct image of currents is the map

$$
\operatorname{trop}_{*}: D^{p, q}(V) \longrightarrow D^{p, q}(U) \otimes_{\mathbb{R}} \mathbb{C}
$$

given by $\operatorname{trop}_{*}(S)(\eta)=S\left(\operatorname{trop}^{*} \eta\right)$ for each $\eta \in A_{c}^{n-p, n-q}(U)$. By linearity, we extend the direct image to a $\mathbb{C}$-linear map trop $*: D(V) \rightarrow D(U) \otimes_{\mathbb{R}} \mathbb{C}$, where $D(U):=\bigoplus_{p, q} D^{p, q}(U)$ is the total space of real Lagerberg currents. 
Lemma 5.1.5 For an open subset $U$ of $N_{\Sigma}, V:=\operatorname{trop}^{-1}(U)$ and $S \in D(V)$, we have the following properties.

(i) $\operatorname{trop}_{*}(S)=\operatorname{trop}_{*}\left(S^{\mathrm{av}}\right)$

(ii) If $S \in D(V)^{\mathbb{S}, F}$, then $\operatorname{trop}_{*}(S) \in D(U) \subset D(U) \otimes_{\mathbb{R}} \mathbb{C}$.

(iii) If $U \subset N_{\mathbb{R}}$ and $S \in D(V)^{\mathbb{S}}$, then $S$ is $F$-invariant if and only if $\operatorname{trop}_{*}(S) \in D(U)$.

Proof Proposition 4.2.3 yields (i). To prove the remaining claims, let $S \in D(V)^{\mathbb{S}}$. Using that $F$ is an involution, we see that (5.2) is equivalent to $\overline{S(\eta)}=(F S)(F \eta)$ for every $\eta \in A_{c}(V)^{\mathbb{S}}$. To prove (ii), we assume that $S$ is $F$-invariant. We have to show $S$ (trop* $\left.\omega\right) \in \mathbb{R}$ for all $\omega \in A_{c}(U)$. Using that $S$ and trop* $\omega$ are $F$-invariant, property (ii) follows from

$$
\overline{\operatorname{trop}_{*} S(\omega)}=\overline{S\left(\operatorname{trop}^{*} \omega\right)}=(F S)\left(F \operatorname{trop}^{*} \omega\right)=S\left(\operatorname{trop}^{*} \omega\right)=\operatorname{trop}_{*} S(\omega) .
$$

To show (iii), let $U \subset N_{\mathbb{R}}$ and assume trop $\tan _{*}(S) \in D(U)$. Then the same computation in reversed order shows $\bar{S}(\eta)=(F S)(F \eta)$ for every $\eta$ of the form trop* $\omega$ with $\omega \in A_{c}(U)$. Using $U \subset N_{\mathbb{R}}$, we deduce from (4.1) and (4.7) that the $\mathbb{C}$-span of such forms is $A_{c}(V)^{\mathbb{S}}$ and hence $\bar{S}(\eta)=(F S)(F \eta)$ holds for all $\eta \in A_{c}(V)^{\mathbb{S}}$ proving $F$-invariance of $S$ and (iii).

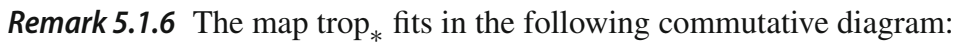

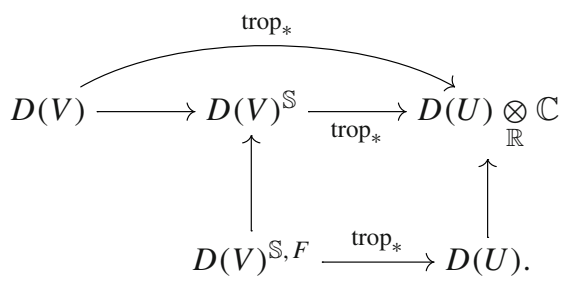

If $U$ is an open subset of the dense orbit $N_{\mathbb{R}}$, then Corollary 4.1 .8 yields that the map

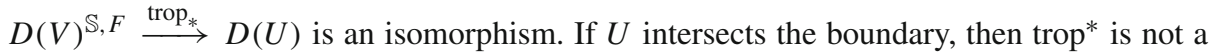
closed immersion and hence trop ${ }_{*}$ is not surjective (see Examples 5.1.11 and 5.1.12 below).

Lemma 5.1.7 Given $\omega \in A^{p, q}(U)$ and $S \in D^{r, s}(V)$, we have the projection formula

$$
\omega \wedge \operatorname{trop}_{*} S=\operatorname{trop}_{*}\left(\operatorname{trop}^{*}(\omega) \wedge S\right) .
$$

Proof Given $\eta \in A_{c}^{n-p-r, n-q-s}(U)$, by Remark 3.2.9(ii) we have

$$
S\left(\operatorname{trop}^{*}(\omega \wedge \eta)\right)=(-1)^{(p+q)(r+s)}\left(\operatorname{trop}^{*}(\omega) \wedge S\right)\left(\operatorname{trop}^{*} \eta\right)
$$

as trop* respects products and (5.3) is an immediate consequence.

Recall that for $\omega \in A^{p, q}(U)$, we have an associated Lagerberg current $[\omega]=\int_{U} \omega \wedge$. in $D^{p, q}(U)$. Similarly, we have a complex current $[\eta] \in D^{p, q}(V)$ associated to a complex form $\eta \in A^{p, q}(V)$. From Lemma 4.2.5 and 5.1.7, we immediately deduce the following result.

Corollary 5.1.8 For every Lagerberg form $\omega \in A^{p, q}(U)$, we have

$$
\operatorname{trop}_{*}\left[\operatorname{trop}^{*}(\omega)\right]=[\omega] \in D^{p, q}(U) .
$$


Definition 5.1.9 Let $V \subset X_{\Sigma}^{\text {an }}$ be an open subset and let $S \in D^{p, p}(V)$ be a current. The current $S$ is called strongly positive (resp. positive, resp. weakly positive) if $S(\eta) \geq 0$ for every weakly positive (resp. positive, resp. strongly positive) form $\eta \in A_{c}^{n-p, n-p}(V)$. The space of weakly positive $(p, p)$-currents will be denoted $D_{w+}^{p, p}(V)$, the space of positive ones by $D_{+}^{p, p}(V)$ and the space of strongly positive ones by $D_{s+}^{p, p}(V)$. The current $S$ is called real if we have $S(\bar{\eta})=\overline{S(\eta)}$ for each $\eta \in A_{c}^{n-p, n-p}(V)$.

Let $U \subset N_{\Sigma}$ be an open subset and let $T \in D^{p, p}(U)$ be a Lagerberg current. The Lagerberg current $T$ is called strongly positive (resp. positive, resp. weakly positive) if it is symmetric and for every weakly positive (resp. positive, resp. strongly positive) Lagerberg form $\eta \in A_{c}^{n-p, n-p}(U)$ the condition $T(\eta) \geq 0$ holds. The space of weakly positive Lagerberg currents of type $(p, p)$ will be denoted $D_{w+}^{p, p}(U)$, the space of positive ones by $D_{+}^{p, p}(U)$, and the space of strongly positive ones by $D_{s+}^{p, p}(U)$.

Lemma 5.1.10 Let $U$ be an open subset of $N_{\Sigma}$ and $V=\operatorname{trop}^{-1}(U) \subset X_{\Sigma}^{\mathrm{an}}$. Every strongly positive current in $D^{p, p}(V)$ (resp. in $D^{p, p}(U)$ ) is positive, every positive current in $D^{p, p}(V)$ (resp. in $D^{p, p}(U)$ ) is weakly positive and every weakly positive current in $D^{p, p}(V)$ (resp. in $D^{p, p}(U)$ ) is real (resp. symmetric). Moreover, if $p=0,1, n-1, n$, then all three positivity notions agree in $D^{p, p}(V)$ (resp. in $\left.D^{p, p}(U)\right)$.

Proof The positivity claims follow from Lemma 4.2.9. By definition, a weakly positive current on $U$ is symmetric. To see that a weakly positive current on $V$ is real, we use Lemma 2.1.4. The latter implies that any real form on $V$ is a real linear combination of strongly positive ones which easily yields the claim.

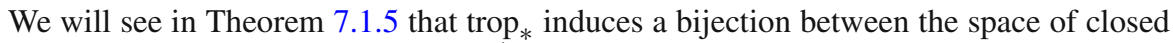
positive complex currents on $V=\operatorname{trop}^{-1}(U)$ that are invariant under $F$ and $\mathbb{S}$ and the space of closed positive Lagerberg currents on $U$. In particular, every closed positive Lagerberg current is in the image of trop ${ }_{*}$. The following two counterexamples show that one can drop neither the closedness assumption nor the positivity assumption. The first example is a positive Lagerberg current and the second example is a closed Lagerberg current, neither of which are in the image of trop ${ }_{*}$.

Example 5.1.11 Let $X_{\Sigma}=\mathbb{A}_{\mathbb{C}}^{1}, N_{\Sigma}=\mathbb{R}_{\infty}, U=\left\{u \in \mathbb{R}_{\infty} \mid u>0\right\}$ and $V=\operatorname{trop}^{-1}(U)=$ $\{z \in \mathbb{C} \mid z \bar{z}<1\}$ as in Example 5.1.1. Let $T: A_{c}^{1,1}(U) \rightarrow \mathbb{R}$ be the linear map

$$
T\left(f d^{\prime} u \wedge d^{\prime \prime} u\right)=\int_{0}^{\infty} e^{x^{2}} f(x) d x .
$$

If $f d^{\prime} u \wedge d^{\prime \prime} u \in A_{c}^{1,1}(U)$, then $f$ has compact support contained in $U \backslash\{\infty\}$. Therefore the integral in (5.4) is finite. To show that $T$ is a continuous functional, we use Proposition 3.2.7. Let $\omega_{n}=g_{n} d^{\prime} u \wedge d^{\prime \prime} u, n \geq 1$ be a sequence of forms in $A_{c}^{1,1}(U)$ that converge to zero. Then there is a neighborhood $V_{0}$ of $\infty$ such that

$$
\left.\omega_{0, n}\right|_{V_{0}}=\pi_{\sigma, 0}^{*}\left(\omega_{\sigma, n}\right)=0
$$

for all $n$, where $\sigma=\mathbb{R}_{\geq 0}$ is the cone corresponding to the point $\infty$. Hence there is a compact $K \subset U \backslash\{\infty\}$ such that $\operatorname{supp}\left(g_{n}\right) \subset K$ for all $K$. Moreover $\left\|g_{n}\right\|_{K}$ converges to zero. Therefore $T\left(\omega_{n}\right)$ also converges to zero. We conclude that $T$ is a Lagerberg current. Clearly it is positive. 
Assume that $S$ is a current on $V$ such that $\operatorname{trop}_{*} S=T$. Let $f_{n}$ and $\varphi_{n}$ be as in Example 5.1.1. Since the functions $\varphi_{n}$ converge to 0 in $A_{c}^{0,0}(V)$, we deduce that

$$
\lim _{n \rightarrow \infty} S\left(\varphi_{n} \frac{i d z \wedge d \bar{z}}{4 \pi z \bar{z}}\right)=0 .
$$

On the other hand, the assumption $\operatorname{trop}_{*} S=T$ yields the desired contradiction as follows:

$$
\begin{aligned}
S\left(\varphi_{n} \frac{i d z \wedge d \bar{z}}{4 \pi z \bar{z}}\right) & =T\left(f_{n} d^{\prime} u \wedge d^{\prime \prime} u\right) \\
& =\int_{0}^{\infty} e^{x^{2}-n^{2}} \rho(x-n) d x>\int_{n}^{n+1} e^{x^{2}-n^{2}} d x>1 .
\end{aligned}
$$

Example 5.1.12 We keep the setting from Example 5.1.11. Then

$$
T(f):=\int_{0}^{\infty} e^{2 e^{x}} f^{\prime}(x) d x .
$$

defines a linear functional $T: A_{c}^{0,0}(U) \rightarrow \mathbb{R}$. Since any $f \in A_{c}^{0,0}(U)$ is constant in a neighborhood of $\infty$, the support of $f^{\prime}$ is a compact subset of $U \backslash\{\infty\}$. As in Example 5.1.11, we see that $T$ defines a current in $D^{1,1}(U)$. This current is closed because it has top degree. We claim that $T$ is not of the form $\operatorname{trop}_{*}(S)$ for any $S \in D^{1,1}(V)$. We argue by contradiction and suppose that $T=\operatorname{trop}_{*}(S)$. Let $\rho$ and $\psi$ be as in Example 5.1.2 and write

$$
\zeta_{n}=\psi(-\log |z|) e^{-1 /|z|} \rho(-\log |z|-n), \quad \zeta=\psi(-\log |z|) e^{-1 /|z|} .
$$

Then $\zeta_{n} \rightarrow \zeta$ in $A_{c}^{0,0}(V)$. Moreover $\zeta_{n}=\operatorname{trop}^{*}\left(f_{n}\right)$ for

$$
f_{n}(x)=\psi(x) e^{-e^{x}} \rho(x-n) \in A_{c}^{0,0}(U) .
$$

Therefore $\lim _{n \rightarrow \infty} S\left(\zeta_{n}\right)=S(\zeta) \in \mathbb{C}$. This contradicts

$$
\left.S\left(\zeta_{n}\right)=T\left(f_{n}\right)=\int_{0}^{\infty} e^{2 e^{x}}\left(\psi(x) e^{-e^{x}} \rho(x-n)\right)\right)^{\prime} d x
$$

converging to $-\infty$ for $n \rightarrow \infty$, as a direct computation using integration by parts shows.

Proposition 5.1.13 Let $S \in D^{p, p}(V)^{\mathbb{S}, F}$. If $S$ is real (resp. positive, resp. weakly positive), then $T:=\operatorname{trop}_{*}(S)$ is symmetric (resp. positive, resp. weakly positive).

Proof Recall from Lemma 5.1.5 that $T \in D^{p, p}(U)$. If $S$ is real, then the Lagerberg current $T$ is symmetric. Indeed for $q:=n-p$ and $\omega \in A_{c}^{q, q}(U)$, we deduce from (4.14) that

$$
T(J(\omega))=S\left(\operatorname{trop}^{*}(J(\omega))\right)=(-1)^{p} S\left(\overline{\operatorname{trop}^{*}(\omega)}\right)=(-1)^{p} \overline{S\left(\operatorname{trop}^{*}(\omega)\right)}=(-1)^{p} T(\omega) .
$$

The positivity statements follow from Lemma 4.2.8.

On the dense orbit, we also have the converse implication.

Lemma 5.1.14 Let $U$ be an open subset of $N_{\mathbb{R}}$, put $V:=\operatorname{trop}^{-1}(U)$, and let $S \in D^{p, p}(V)^{\mathbb{S}, F}$ be an invariant current such that $\operatorname{trop}_{*}(S)$ is a positive current on $U$. Then $S$ is a positive current on $V$. 
Proof Given a positive form $\alpha \in A_{c}^{n-p, n-p}(V)$, the averaged form $\alpha^{\text {av }} \in A_{c}^{p, p}(V)^{\mathbb{S}}$ from Remark 4.1.1 is again positive. From Lemma 2.3.3, we get that the invariant form

$$
\alpha^{\text {inv }}=\left(\alpha^{\mathrm{av}}+F\left(\alpha^{\mathrm{av}}\right)\right) / 2 \in A_{c}^{n-p, n-p}(V)^{\mathbb{S}, F}
$$

is positive as well. Since $U \subset N_{\mathbb{R}}$, we conclude by Proposition 4.1.7 that $\alpha^{\text {inv }}=\operatorname{trop}^{*}(\beta)$ for a uniquely determined Lagerberg form $\beta$ in $A_{c}^{p, p}(U)$. Finally $\beta$ is positive by Lemma 4.1.12 and $S(\alpha)=S\left(\alpha^{\text {inv }}\right)=\operatorname{trop}_{*}(S)(\beta) \geq 0$ shows our claim.

We will see in the next subsection that positive currents have measure coefficients. The key result to prove this is captured in the following proposition. We refer the reader to Appendix A for the convention we use about Radon measures.

Proposition 5.1.15 Let $U$ be either an open subset of $X_{\Sigma}^{\text {an }}$ or of $N_{\Sigma}$. Let

$$
T: A_{c}^{0,0}(U) \longrightarrow \mathbb{R}
$$

be a linear functional such that $T(f) \geq 0$ for every non-negative $f \in A_{c}^{0,0}(U)$. Then there is a unique positive Radon measure $\mu$ on $U$ with $T(f)=\int_{U} f d \mu$ for every $f \in A_{c}^{0,0}(U)$.

Proof The following argument works for the complex and the Lagerberg case.

Let $K$ be a compact subset of $U$ and let $C_{K}^{0}(U, \mathbb{R})$ be the set of continuous real functions with support in $K$. As usual, this real vector space is endowed with the supremum norm \|\|$_{\text {sup }}$. We also consider the subspace $A_{K}^{0,0}(U, \mathbb{R})$ of smooth real functions with support in $K$ and its subspace $A_{K}^{0,0}\left(U, \mathbb{R}_{\geq 0}\right)$ of non-negative functions.

We claim that the restriction of $T$ to $A_{K}^{0,0}(U, \mathbb{R})$ is continuous with respect to the supnorm. Since we have smooth partitions of unity by Proposition 3.2.12, there is a non-negative $\chi_{K} \in A_{c}^{0,0}(U)$ with $\chi_{K}(x)=1$ for all $x \in K$. Now the positivity of $T$ yields

$$
-T\left(\chi_{K}\right)\|f\|_{\text {sup }} \leq T(f) \leq T\left(\chi_{K}\right)\|f\|_{\text {sup }}
$$

for all smooth functions $f$ with compact support in $K$. This proves the claim.

Let $f \in C_{c}^{0}(U, \mathbb{R})$. To define $T(f)$, we use Corollary 3.2.13 or its classical analogue to get a sequence $\left(f_{n}\right)_{n \in \mathbb{N}}$ in $A_{c}^{0,0}(U, \mathbb{R})$ which converges to $f$. It follows from the proof of that corollary as well as from Proposition 3.2.6 that we can find a compact subset $K$ of $U$ such that $f_{n} \in A_{K}^{0,0}(U, \mathbb{R})$ for all $n \in \mathbb{N}$. The sequence $\left(f_{n}\right)_{n \in \mathbb{N}}$ converges to $f$ with respect to the sup-norm on $K$. By the continuity of $T$ shown above, it is clear that $T\left(f_{n}\right)$ converges to a real number. The limit $T(f)$ neither depends on the choice of the sequence nor on the choice of $K$. Note also that if $f \geq 0$, then $\chi_{K} \cdot\left(f_{n}+\left\|f_{n}-f\right\|_{\text {sup }}\right)$ is a sequence in $C_{\operatorname{supp}\left(\chi_{K}\right)}^{0}\left(U, \mathbb{R}_{\geq 0}\right)$ converging to $f$ with respect to the sup-norm. We conclude easily that the above defines a positive linear functional $T$ on $C_{c}^{0}(U, \mathbb{R})$ which extends the given functional. This is equivalent to have a positive Radon measure $\mu$ on $U$ with $T(f)=\int_{U} f d \mu$ for every $f \in C_{c}^{0}(U, \mathbb{R})$.

Note that uniqueness is obvious as a positive linear functional on $C_{c}^{0}(U, \mathbb{R})$ is continuous on $C_{K}^{0}(U, \mathbb{R})$ (by the same argument as above) and so our definition of $T(f)$ is forced.

We can now prove that positive $(n, n)$-currents on open subsets of $X_{\Sigma}^{\text {an }}$ or $N_{\Sigma}$ are the same as positive Radon measures.

Proposition 5.1.16 Let $U$ be either an open subset of $X_{\Sigma}^{\mathrm{an}}$ or of $N_{\Sigma}$. For every positive current $T \in D^{n, n}(U)$, there is a unique positive Radon measure $\mu$ on $U$ such that

$$
T(f)=\int_{U} f d \mu \quad \forall f \in A_{c}^{0,0}(U) .
$$


Conversely, every positive Radon measure $\mu$ on $U$ induces a current $T \in D_{+}^{n, n}(U)$ by (5.6).

Proof If $T$ is a positive current in $D^{n, n}(U)$, then it is a positive linear functional on $A_{c}^{0,0}(U)$. Hence the first claim follows from Proposition 5.1.15. Conversely, we have seen in Example 3.2.11 that every real Radon measure $\mu$ induces an element $T \in D^{n, n}(U)$ with (5.6). Clearly, if $\mu$ is positive, then $T$ is positive as well.

The following result is a prototype for our main correspondence result in Theorem 7.1.5.

Corollary 5.1.17 Let $U$ be an open subset of $N_{\Sigma}$ and let $V:=\operatorname{trop}^{-1}(U)$. Then the map trop $_{*}$ induces a linear isomorphism $D^{n, n}(V)_{+}^{\mathbb{S}} \rightarrow D^{n, n}(U)_{+}$of real cones.

Proof Since $N_{\Sigma}=X_{\Sigma}^{\text {an }} / \mathbb{S}$ (see Remark 3.1.3), it is clear that trop* induces an isomorphism between $C_{c}^{0}(U, \mathbb{R})$ and the subspace of $C_{c}^{0}(V, \mathbb{R})$ given by the $\mathbb{S}$-invariant functions. Hence trop $_{*}$ maps the cone of $\mathbb{S}$-invariant positive Radon measures on $V$ isomorphically onto the cone of positive Radon measures on $U$. By Proposition 5.1.16, we get the claim.

\subsection{Co-coefficients of currents}

To extend Proposition 5.1.15 to $(p, p)$-currents, we place ourselves in the local setting. We assume in this subsection that $N=\mathbb{Z}^{n}$ and that $\Sigma$ is the fan given by the maximal cone $\mathbb{R}_{\geq 0}^{n}$ and its faces in $N_{\mathbb{R}}=\mathbb{R}^{n}$. Then $N_{\Sigma}=\mathbb{R}_{\infty}^{n}$ and $X_{\Sigma}$ is the smooth toric variety $\mathbb{A}_{\mathbb{C}}^{n}$. Recall that any smooth toric variety can be covered by toric affine varieties isomorphic to open subvarieties of this one. The splitting $N=\mathbb{Z}^{n}$ induces coordinates $\left(z_{1}, \ldots, z_{n}\right)$ in $X_{\Sigma}^{\text {an }}$ and $\left(u_{1}, \ldots, u_{n}\right)$ in $N_{\mathbb{R}}$.

Definition 5.2.1 Let $V \subset X_{\Sigma}^{\text {an }}=\mathbb{C}^{n}$ be an open subset and let $S \in D^{p, p}(V)$, write $q:=n-p$ and let $I, J \subset\{1, \ldots, n\}$ with $|I|=|J|=q$. Then the co-coefficient $S^{I J}$ of $S$ is the distribution on $V$ defined by

$$
S^{I J}(f)=S\left(i^{q^{2}} f d z_{I} \wedge d \bar{z}_{J}\right) .
$$

For $I \subset\{1, \ldots, n\}$, we consider the following union of strata

$$
E^{I}:=\bigcup_{i \in I}\left\{u_{i}=\infty\right\} \subset N_{\Sigma}=\mathbb{R}_{\infty}^{n} .
$$

Definition 5.2.2 Let $U \subset N_{\Sigma}=\mathbb{R}_{\infty}^{n}$ be open and $T \in D^{p, p}(U)$. Let $q, I, J$ be as above. Then the co-coefficient $T^{I J}$ is the Lagerberg distribution on $U \backslash E^{I \cup J}$ defined by

$$
T^{I J}(f)=T\left((-1)^{\frac{q(q-1)}{2}} f d^{\prime} u_{I} \wedge d^{\prime \prime} u_{J}\right)
$$

Remark 5.2.3 Note that we define $T^{I J} \in D^{n, n}\left(U \backslash E^{I \cup J}\right)$ because, by definition of Lagerberg forms, we can only plug in functions that vanish in a neighborhood of $E^{I \cup J}$.

Remark 5.2.4 Conversely, given $S^{I J} \in D^{n, n}(V)$ for all $I, J \subset\{1, \ldots, n\}$ with $|I|=|J|=$ $q=n-p$, there is a unique complex current $S \in D^{p, p}(V)$ with co-coefficients $S^{I J}$.

Similarly, one can show that given $T^{I J} \in D^{n, n}\left(U \backslash E^{I \cup J}\right)$ for all $I, J \subset\{1, \ldots, n\}$ with $|I|=|J|=q=n-p$, there is a unique $T \in D^{p, p}(U)$ with co-coefficients $T^{I J}$.

Positive currents have measure co-coefficients, instead of just distribution co-coefficients. The complex case is given by [9, Proposition III.1.14]: 
Proposition 5.2.5 Let $V \subset X_{\Sigma}^{\text {an }}=\mathbb{C}^{n}$ and $S \in D^{p, p}(V)$ be a weakly positive current. Then the co-coefficients $S^{I J}$ are complex Radon measures on $V$ that satisfy $\overline{S^{I J}}=S^{J I}$ for all multi-indices $I, J$ with $|I|=|J|=q$. Moreover, all $S^{I I}$ are positive Radon measures and the total variation measures $\left|S^{I J}\right|$ satisfy the estimates

$$
\lambda_{I} \lambda_{J}\left|S^{I J}\right| \leq 2^{q} \sum_{M} \lambda_{M}^{2} S^{M M}, \quad I \cap J \subset M \subset I \cup J,
$$

for any collection of real numbers $\lambda_{k} \geq 0, k=1, \ldots, n$, and $\lambda_{I}=\prod_{k \in I} \lambda_{k}$.

We have seen in Example 2.3.6 that in the tropical case it is reasonable to restrict our attention to positive Lagerberg forms and hence to positive Lagerberg currents, as opposed to weakly positive currents.

Proposition 5.2.6 Let $U \subset N_{\Sigma}=\mathbb{R}_{\infty}^{n}$ be an open subset and $T \in D^{p, p}(U)$ a positive current. Then the co-coefficients $T^{I J}$ are real Radon measures on $U \backslash E^{I \cup J}$ that satisfy $T^{I J}=T^{J I}$ for all multi-indices $I, J$ with $|I|=|J|=q$. Moreover, all $T^{I I}$ are positive Radon measures and the total variation measures $\left|T^{I J}\right|$ satisfy the estimates

$$
\lambda_{I} \lambda_{J}\left|T^{I J}\right| \leq \frac{1}{2}\left(\lambda_{I}^{2} T^{I I}+\lambda_{J}^{2} T^{J J}\right)
$$

for any pair of real numbers $\lambda_{I}, \lambda_{J} \geq 0$.

Proof By definition, Lagerberg currents are real valued. The condition

$$
T^{I J}=T^{J I}
$$

follows from the symmetry of $T$. For every $f \in A_{c}^{0,0}\left(U \backslash E^{I \cup J}\right)$, we have

$$
\left(T^{I J}+T^{J I}\right)(f)=T\left(f \cdot(-1)^{\frac{q(q-1)}{2}}\left(d^{\prime} u_{I} \wedge d^{\prime \prime} u_{J}+d^{\prime} u_{J} \wedge d^{\prime \prime} u_{I}\right)\right) .
$$

Moreover $(-1)^{\frac{q(q-1)}{2}}\left(d^{\prime} u_{I} \wedge d^{\prime \prime} u_{J}+d^{\prime} u_{J} \wedge d^{\prime \prime} u_{I}\right)$ can be written as the difference of two positive Lagerberg forms

$$
\begin{aligned}
(-1)^{\frac{q(q-1)}{2}}\left(d^{\prime} u_{I} \wedge d^{\prime \prime} u_{J}+d^{\prime} u_{J} \wedge d^{\prime \prime} u_{I}\right)= & (-1)^{\frac{q(q-1)}{2}}\left(d^{\prime} u_{I}+d^{\prime} u_{J}\right) \wedge\left(d^{\prime \prime} u_{I}+d^{\prime \prime} u_{J}\right) \\
& -(-1)^{\frac{q(q-1)}{2}}\left(d^{\prime} u_{I} \wedge d^{\prime \prime} u_{I}+d^{\prime} u_{J} \wedge d^{\prime \prime} u_{J}\right) .
\end{aligned}
$$

Since the product of a positive Lagerberg current by a positive Lagerberg form is a positive Lagerberg current by Remark 2.2.2, we deduce from equations (5.10), (5.11) and from Proposition 5.1.15 that $T^{I J}+T^{J, I}$ is a real Radon measure on $U \backslash E^{I \cup J}$. From equation (5.9), it follows that $T^{I J}$ is also a real Radon measure on $U \backslash E^{I \cup J}$. In the special case $I=J$, there is no need for (5.11) and we can directly deduce from the definition of the co-coefficient $T^{I I}$ or from (5.10) that $T^{I I}$ is a positive Radon measure on $U \backslash E^{I}$.

Note that for every positive continuous function with compact support contained in $U \backslash$ $E^{I \cup J}$ and for every pair of non-negative real numbers $\lambda_{I}, \lambda_{J}$, we have the inequalities

$$
T\left(f \cdot(-1)^{\frac{q(q-1)}{2}}\left(\lambda_{I} d^{\prime} u_{I} \pm \lambda_{J} d^{\prime} u_{J}\right) \wedge\left(\lambda_{I} d^{\prime \prime} u_{I} \pm \lambda_{J} d^{\prime \prime} u_{J}\right)\right) \geq 0 .
$$

Expanding and using equation (5.9), we deduce

$$
\pm 2 \lambda_{I} \lambda_{J} T^{I J}(f) \leq \lambda_{I}^{2} T^{I, I}(f)+\lambda_{J}^{2} T^{J, J}(f),
$$

from which Eq. (5.8) follows. 
Observe that (5.8) is stronger than (5.7). This is because in Proposition 5.2.6 we are dealing with positive currents while Proposition 5.2.5 deals with weakly positive currents.

Example 5.2.7 We show that there can be no analogue of Proposition 5.2.6 for weakly positive Lagerberg currents.

Example 2.3.6 gives a non-zero Lagerberg form $\omega$ of type $(2,2)$ on $\mathbb{R}^{4}$ such that $\pm \omega$ are both weakly positive. In fact $\omega$ was constructed such that $\omega \wedge \eta=0$ for each strongly positive Lagerberg form $\eta$ of type $(2,2)$. By definition, the associated Lagerberg currents $\pm[\omega]$ are weakly positive. We compute the co-coefficients of $T=[\omega]$. For $I=\{3,4\}, J=\{1,2\}$ and $f \in C_{c}^{\infty}\left(N_{\mathbb{R}}\right)$, we have

$$
T^{J I}(f)=T^{I J}(f)=-T\left(f d^{\prime} u_{3} \wedge d^{\prime} u_{4} \wedge d^{\prime \prime} u_{1} \wedge d^{\prime \prime} u_{2}\right)=\int_{\mathbb{R}^{4}} f(u) d u_{1} d u_{2} d u_{3} d u_{4}
$$

by using the formula for $\omega$ in Example 2.3.6. We conclude that $T^{J I}=T^{I J}$ is the Lebesgue measure on $\mathbb{R}^{4}$ and the same holds for $I=\{2,3\}$ and $J=\{1,4\}$. On the other hand, for $I=\{2,4\}, J=\{1,3\}$, we have that $-T^{J I}=-T^{I J}$ agrees with the Lebesgue measure on $\mathbb{R}^{4}$. All other co-coefficients are 0 . In particular, for all $M \subset\{1,2,3,4\}$ with two elements, we have $T^{M M}=0$. We conclude that no estimate of the form (5.8) or (5.7) holds.

In Example 5.2.7, all co-coefficients were positive or negative Radon measures on $\mathbb{R}^{4}$.

Example 5.2.8 We construct from $\omega$ in Example 2.3.6 a weakly positive Lagerberg current $T$ whose co-coefficients are not all Radon measures.

Since $\omega \wedge \alpha=0$ for all strongly positive forms $\alpha$, we have that $\omega \wedge T^{\prime}$ is weakly positive for every symmetric current $T^{\prime}$ of type $(0,0)$. This already shows that it is very unlikely that every weakly positive current has measure coefficients. Indeed, one may check that for the Lagerberg currents

$$
T^{\prime}: A_{c}^{4,4}\left(\mathbb{R}^{4}\right) \longrightarrow \mathbb{R}, T^{\prime}\left(f d^{\prime} u_{1} \wedge d^{\prime \prime} u_{1} \wedge \ldots \wedge d^{\prime} u_{4} \wedge d^{\prime \prime} u_{4}\right)=\frac{\partial f}{\partial u_{1}}(0,0,0,0)
$$

and $T=\omega \wedge T^{\prime}$, and the sets $I=\{3,4\}$ and $J=\{1,2\}$, we have

$$
T^{I J}(f)=\frac{\partial f}{\partial u_{1}}(0,0,0,0)
$$

It is well-known that uniform convergence does not imply convergence of derivatives. Therefore the co-coefficient $T^{I J}$ is not a Radon measure.

\section{Tropicalization of positive currents}

We consider a smooth fan $\Sigma$ in $N_{\mathbb{R}}$ with associated complex toric variety $X_{\Sigma}$ and corresponding tropicalization $N_{\Sigma}$. In this section, we will describe precisely which positive Lagerberg

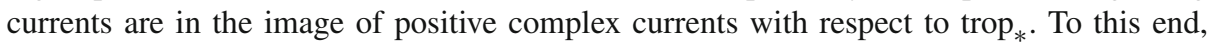
in the first subsection, we will introduce a canonical decomposition of positive currents in the complex and in the Lagerberg case and use it to give the desired characterization in the second subsection. 


\subsection{Decomposition of positive currents along the strata}

In this subsection, we give a canonical decomposition of a positive current along the boundary strata. As usual, we handle the complex and the tropical case simultaneously. For simplicity, we will often give the arguments only in the case of Lagerberg currents as the complex case is completely similar and even easier as the exceptional sets $E^{I}$ for co-coefficients do not occur (see Sect. 5.2). At the end of the subsection, we will study functoriality of the canonical decomposition with respect to trop ${ }_{*}$.

Remark 6.1.1 Recall that the torus action of $\mathbb{T}$ yields stratifications of the toric variety

$$
X_{\Sigma}=\coprod_{\sigma \in \Sigma} O(\sigma) \text { and } X_{\Sigma}^{\mathrm{an}}=\coprod_{\sigma \in \Sigma} O(\sigma)^{\mathrm{an}}
$$

into locally closed subsets given by the orbits $O(\sigma)$. Similarly, we have the stratification

$$
N_{\Sigma}=\coprod_{\sigma \in \Sigma} N(\sigma)
$$

given in Definition 3.1.1. Note that the stratification of $N_{\Sigma}$ induces the one of $X_{\Sigma}^{\text {an }}$ by $O(\sigma)^{\text {an }}=\operatorname{trop}^{-1}(N(\sigma))$ for any $\sigma \in \Sigma$. For an open subset $V$ of $X_{\Sigma}^{\text {an }}$ and an open subset $U$ of $N_{\Sigma}$, we get induced stratifications

$$
V=\coprod_{\sigma \in \Sigma} V \cap O(\sigma)^{\text {an }} \text { and } U=\coprod_{\sigma \in \Sigma} U \cap N(\sigma) .
$$

For any $\rho \in \Sigma$, the open subset $\bigsqcup_{\nu \prec \rho} O(\nu)$ of $X_{\Sigma}$ is the affine toric variety associated with $\rho$ and its tropicalization $\bigsqcup_{\nu \prec \rho} N(v)$ is open in $N_{\Sigma}$. This gives rise to the open subsets

$$
V_{\rho}:=\coprod_{\nu \prec \rho} V \cap O(v)^{\text {an }} \text { and } U_{\rho}:=\coprod_{\nu \prec \rho} U \cap N(v)
$$

of $V$ and $U$, respectively. For varying $\rho \in \Sigma$, these open subsets cover $V$ (resp. $U$ ).

Definition 6.1.2 Since $\Sigma$ is smooth, a given cone $\rho$ in $\Sigma$ is generated by part of a $\mathbb{Z}$-basis $b_{1}, \ldots, b_{n}$ of $N$. Using the corresponding coordinates, we may view $V_{\rho}$ (resp. $U_{\rho}$ ) as an open subset of $\mathbb{C}^{n}$ (resp. $\mathbb{R}_{\infty}^{n}$ ). We call such an identification of $V_{\rho}$ (resp. $U_{\rho}$ ) with an open subset of $\mathbb{C}^{n}$ (resp. $\mathbb{R}_{\infty}^{n}$ ) a choice of toric coordinates on $V_{\rho}$ (resp. $\left.U_{\rho}\right)$. We usually denote the corresponding complex coordinates by $z_{1}, \ldots, z_{n}$ and the corresponding tropical coordinates by $u_{1}, \ldots, u_{n}$.

Note that for a current $T$ of bidegree $(p, q)$ on $V$ (resp. $U$ ), the choice of toric coordinates on $V_{\rho}$ (resp. $U_{\rho}$ ) leads to well-defined co-coefficients $\left(\left.T\right|_{V_{\rho}}\right)^{I J}$ (resp. $\left(\left.T\right|_{U_{\rho}}\right)^{I J}$ ) for all $I, J \subset\{1, \ldots, n\}$ with $|I|=|J|=q=n-p$. Recall that, if $T$ is positive, then in the complex case the co-coefficient $\left(\left.T\right|_{V_{\rho}}\right)^{I J}$ is a Radon measure on the open set $V_{\rho}$ while in the Lagerberg case $\left(\left.T\right|_{U_{\rho}}\right)^{I J}$ is a Radon measure on the open set $U_{\rho} \backslash E^{I \cup J}$ (see Sect. 5.2). This allows us to define the notion of a null set of a positive current.

Definition 6.1.3 Let $U$ be an open subset of $X_{\Sigma}^{\text {an }}$ (resp. $N_{\Sigma}$ ). We say that $T \in D^{p, p}(U)$ has measure co-coefficients if for every $\rho \in \Sigma$ and some choice of toric coordinates on $U_{\rho}$, the co-coefficients $T^{I J}$ are complex (resp. real) Radon measures on $U_{\rho}$ (resp. on $U_{\rho} \backslash E^{I \cup J}$ ).

Let $T \in D^{p, p}(U)$ have measure co-coefficients and let $A$ be a Borel subset of $U$. We say that $A$ is a null set for $T$ if, for all $\rho \in \Sigma$ and some choice of toric coordinates on $U_{\rho}$, the set $A \cap U_{\rho}$ (resp. $A \cap U_{\rho} \backslash E^{I \cup J}$ ) is a null set of the total variation measure $\left|\left(\left.T\right|_{V_{\rho}}\right)^{I J}\right|$ (resp. $\left|\left(\left.T\right|_{U_{\rho}}\right)^{I J}\right|$ ) for all $I, J \subset\{1, \ldots, n\}$ with $|I|=n-p$. 
Using change of variables, it is easy to see that the conditions in Definition 6.1.3 do not depend on the choice of toric coordinates.

Lemma 6.1.4 Let $U$ be open in $X_{\Sigma}^{\text {an }}$ (resp. in $N_{\Sigma}$ ) and let $A \subset U$ be closed in $U$. Then there is an increasing sequence of smooth functions $\psi_{k} \geq 0$ in $A_{c}^{0,0}(U \backslash A)$ which converges pointwise to the characteristic function of $U \backslash A$.

For any $\alpha \in A_{c}^{p, q}(U)$, the forms $\alpha_{k}:=\psi_{k} \alpha \in A_{c}^{p, q}(U)$ have compact support in $U \backslash A$. If $\alpha$ is a positive form, then $\alpha_{k}$ and $\alpha-\alpha_{k}$ are both positive.

For every $T \in D^{n-p, n-q}(U)$ with measure co-coefficients, we have:

(a) if $A$ is a null set for $T$, then $\lim _{k \rightarrow \infty} T\left(\alpha_{k}\right)=T(\alpha)$;

(b) if $U \backslash A$ is a null set for $T$, then $\lim _{k \rightarrow \infty} T\left(\alpha_{k}\right)=0$.

Proof Let $\alpha \in A_{c}^{p, q}(U)$ with support contained in the compact subset $K$ of $U$. Since $U$ is a metric space and since $A \cap K$ is compact, there is a strictly decreasing sequence $\left(W_{k}\right)_{k \in \mathbb{N}}$ of relatively compact open subsets of $U$ with

$$
\bigcap_{k \in \mathbb{N}} W_{k}=A \cap K \quad \text { and } \quad W_{0} \supset \overline{W_{1}} \supset W_{1} \supset \overline{W_{2}} \supset \cdots .
$$

Now a partition of unity (see Proposition 3.2.12 for the Lagerberg case) gives the existence of a function $\varphi_{k} \in A_{c}^{0,0}\left(W_{k}\right)$ with $0 \leq \varphi_{k} \leq 1$ and $\varphi_{k} \equiv 1$ on $W_{k+1}$ for every $k \in \mathbb{N}$. We note that the $\varphi_{k}$ form a decreasing sequence of smooth functions with compact support which converges pointwise to the characteristic function of $A \cap K$. For the sequence $\varphi_{k}:=1-\psi_{k}$, we get the first claim. Evaluating $T\left(\alpha_{k}\right)$ in terms of the co-coefficients $T^{I J}$ and using that the latter are Radon measures, we deduce (a) and (b) from the monotone convergence theorem. If $\alpha$ is positive, then every $\alpha_{k}=\psi_{k} \alpha$ and every $\alpha-\alpha_{k}=\varphi_{k} \alpha$ is positive.

Remark 6.1.5 In the complex case of Lemma 6.1.4, if $A$ is $\mathbb{S}$-invariant, then we can choose the smooth functions $\psi_{k}$ to be $\mathbb{S}$-invariant. Indeed, this follows easily from Lemma 6.1.4 by averaging the $\psi_{k}$ with respect to the Haar probability measure on $\mathbb{S}$.

The decomposition theorem is the following result.

Theorem 6.1.6 Let $U$ be an open subset of $X_{\Sigma}^{\mathrm{an}}$ (resp. of $N_{\Sigma}$ ) and let $T$ be a positive current in $D^{p, p}(U)$. Then there is a unique decomposition

$$
T=\sum_{\sigma \in \Sigma} T_{\sigma}
$$

such that, for every $\sigma \in \Sigma$, the following two conditions are satisfied

(i) $T_{\sigma} \in D^{p, p}(U)$ is positive;

(ii) the set $U \backslash O(\sigma)$ (resp. $U \backslash N(\sigma)$ ) is a null set for $T_{\sigma}$.

Moreover, the support of the current $T_{\sigma}$ is contained in $V \cap \overline{O(\sigma)^{\mathrm{an}}}($ resp. $U \cap \overline{N(\sigma)})$.

We call (6.1) the canonical decomposition of the positive current $T$.

Proof We write the proof only in the Lagerberg case as the complex case is analogous. The statement about the support is a direct consequence of statement (ii) so we only need to prove the existence and uniqueness of the decomposition.

Using a partition of unity provided by Proposition 3.2.12, the existence of such decomposition can be checked locally. Moreover, since currents form a sheaf, the unicity can also 
be checked locally. Therefore we choose a cone $\rho \in \Sigma$ and replace $U$ by $U_{\rho}$. We also make a choice of toric coordinates, so we can assume from now on that $U \subset \mathbb{R}_{\infty}^{n}$.

For each pair of subsets $I, J$ with $|I|=|J|=p$ we write

$$
T^{I J}=T_{+}^{I J}-T_{-}^{I J}
$$

with $T_{ \pm}^{I J}$ the positive and the negative part of the Radon measure $T^{I J}$. We denote by $\mu_{ \pm}^{I J}$ the corresponding Borel measures. For any $\sigma \in \Sigma$, let us consider the immersion

$$
i_{\sigma}^{I J}: U \cap N(\sigma) \backslash E^{I \cup J} \longrightarrow U \backslash E^{I \cup J} .
$$

We define a new Borel measure $\mu_{ \pm, \sigma}^{I J}$ on $U \backslash E^{I \cup J}$ by first restricting $\mu_{ \pm}^{I J}$ to the locally closed subset $U \cap N(\sigma) \backslash E^{I \cup J}$ and then using the image measure with respect to $i_{\sigma}^{I J}$. Since the sets $N(\sigma)$ form a stratification of $N_{\Sigma}$, we get the decomposition

$$
\mu_{ \pm}^{I J}=\sum_{\sigma \in \Sigma} \mu_{ \pm, \sigma}^{I J}
$$

of Borel measures. It follows that $\mu_{ \pm, \sigma}^{I J} \leq \mu_{ \pm}^{I J}$. Note that in our setting, the Radon measures correspond to locally finite Borel measures (see Appendix A). We conclude that $\mu_{ \pm, \sigma}^{I J}$ is a locally finite Borel measure and hence we get a real Radon measure $T_{\sigma}^{I J}$ on $U \backslash E^{I \cup J}$ corresponding to $\mu_{+, \sigma}^{I J}-\mu_{-, \sigma}^{I J}$. By Remark 5.2.4, we obtain a unique current $T_{\sigma} \in D^{p, p}(U)$ with co-coefficients $T_{\sigma}^{I J}$. Note that $T_{\sigma}$ has measure co-coefficients. By construction, the set $U \backslash\left(E^{I \cup J} \cup N(\sigma)\right)$ is a null set with respect to the Borel measure $\mu_{+, \sigma}^{I J}+\mu_{-, \sigma}^{I J}$ associated to the total variation measure $\left|T_{\sigma}\right|$. The decomposition $T=\sum_{\sigma} T_{\sigma}$ follows from (6.2). It remains to show uniqueness and property (i).

We prove uniqueness of $T_{\sigma}$ in the decomposition (6.1) by induction with respect to the partial ordering $\prec$ on $\Sigma$. We take $\sigma \in \Sigma$ and we suppose that uniqueness of $T_{\tau}$ is known for all $\tau \neq \sigma$ with $\tau \prec \sigma$. Recall from Remark 6.1.1 that $U_{\sigma}$ is an open subset of $U$ and that $A_{\sigma}:=U \backslash U_{\sigma}$ is the closed subset of $U$ given by the disjoint union of all $N(\tau) \cap U$ with $\tau \in \Sigma$ not a face of $\sigma$. For $\alpha \in A_{c}^{n-p, n-p}(U)$, Lemma 6.1 .4 gives a sequence $\left(\alpha_{k}\right)_{k \in \mathbb{N}}$ in $A_{c}^{n-p, n-p}(U)$ with

$$
\lim _{k \rightarrow \infty} T_{\tau}\left(\alpha_{k}\right)=T_{\tau}(\alpha)
$$

for $\tau \prec \sigma$ and

$$
\lim _{k \rightarrow \infty} T_{\tau}\left(\alpha_{k}\right)=0
$$

for all $\tau \in \Sigma$ which are not faces of $\sigma$. Now the decomposition (1.6) gives

$$
\lim _{k \rightarrow \infty} T\left(\alpha_{k}\right)=\sum_{\tau \prec \sigma} T_{\tau}(\alpha)
$$

and uniqueness of $T_{\sigma}$ follows from our induction hypotheses.

For the positivity (i), we prove first that the current

$$
T_{\sigma}^{\prime}:=T-\sum_{\tau \prec \sigma, \tau \neq \sigma} T_{\tau}
$$

is positive. For this let now $A_{\sigma}^{\prime}=A_{\sigma} \cup(N(\sigma) \cap U)$ be the union of all $N(\tau) \cap U$ with $\tau$ ranging over all cones of $\Sigma$ which are not proper faces of $\sigma$. Clearly, $A_{\sigma}^{\prime}$ is also a closed subset of $U$. Let $\alpha^{\prime} \in A_{c}^{n-p, n-p}(U)$ be positive. Then Lemma 6.1.4 gives a sequence $\left(\alpha_{k}^{\prime}\right)_{k \in \mathbb{N}}$ 
in $A_{c}^{n-p, n-p}(U)$ with both $\alpha_{k}^{\prime}$ and $\alpha^{\prime}-\alpha_{k}^{\prime}$ positive. Using the properties (a) and (b) of the sequence from Lemma 6.1.4, the same argument as before shows that

$$
\lim _{k \rightarrow \infty} T\left(\alpha_{k}^{\prime}\right)=\sum_{\tau \prec \sigma, \tau \neq \sigma} T_{\tau}\left(\alpha^{\prime}\right)
$$

and hence

$$
T_{\sigma}^{\prime}\left(\alpha^{\prime}\right)=\lim _{k \rightarrow \infty} T\left(\alpha^{\prime}-\alpha_{k}^{\prime}\right) .
$$

Since $T$ and all $\alpha^{\prime}-\alpha_{k}^{\prime}$ are positive, we deduce that $T_{\sigma}^{\prime}$ is positive.

To prove that $T_{\sigma}$ is positive, we consider again the closed subset $A_{\sigma}$. Let $\alpha \in A_{c}^{n-p, n-p}(U)$ be positive. By Lemma 6.1.4, there is a positive sequence $\left(\alpha_{k}\right)_{k \in \mathbb{N}}$ in $A_{c}^{n-p, n-p}(U)$ such that

$$
\lim _{k \rightarrow \infty} T_{\sigma}\left(\alpha_{k}\right)=T_{\sigma}(\alpha) \quad \text { and } \quad \lim _{k \rightarrow \infty} T_{\tau}\left(\alpha_{k}\right)=0
$$

for all $\tau \in \Sigma$ which are not faces of $\sigma$. We conclude that

$$
\lim _{k \rightarrow \infty} T_{\sigma}^{\prime}\left(\alpha_{k}\right)=T_{\sigma}(\alpha)
$$

Using that $T_{\sigma}^{\prime}\left(\alpha_{k}\right) \geq 0$ by the positivity of $T_{\sigma}^{\prime}$ and $\alpha_{k}$, we deduce that $T_{\sigma}$ is positive.

In the complex case, we will show next that the canonical current $T_{\sigma}$ from the canonical decomposition (6.1) is not always the push-forward of a current on $V \cap \overline{O(\sigma)}^{\text {an }}$.

Example 6.1.7 Let us consider $V=X_{\Sigma}^{\text {an }}=\mathbb{C}^{2}$ where $\Sigma$ is the fan whose maximal cone is the positive quadrant in $\mathbb{R}^{2}$ and let $T \in D^{1,1}\left(\mathbb{C}^{2}\right)$ be given by

$$
T\left(\sum_{k, l} \alpha_{k l} i d z_{k} \wedge d \bar{z}_{l}\right)=\alpha_{1,1}(0,1) .
$$

Using that the coefficients $\alpha_{k k}$ of a positive $(1,1)$-form are non-negative, we see that $T$ is a positive current. We conclude that $T=T_{\sigma}$ for the cone $\sigma$ generated by $(1,0)$. Note that the push-forward $S$ of a current on the stratum closure $\overline{O(\sigma)}^{\text {an }}=\left\{z_{1}=0\right\}$ can only have a nonzero co-coefficient $S^{2,2}$. Since all the co-coefficients of $T$ are zero except $T^{1,1}$ which is given by the Dirac measure $\delta_{(1,0)}$ in the point $(1,0)$, we conclude that $T_{\sigma}$ is not the push-forward of a current on $\overline{O(\sigma)}^{\text {an }}$.

Note that the analogue of Example 6.1.7 in the Lagerberg case gives no counterexample as $T$ is zero in this case since the point $(\infty, 0)$ belongs to $E^{\{1\}}$. This is explained by the following result.

Proposition 6.1.8 Let $U$ be an open subset of $X_{\Sigma}^{\mathrm{an}}$ (resp. $N_{\Sigma}$ ). Let $T$ be a positive current of bidegree $(p, p)$ on $U$ with canonical decomposition $T=\sum_{\sigma \in \Sigma} T_{\sigma}$. Let us fix $\sigma \in \Sigma$.

(a) If $U \subset X_{\Sigma}^{\mathrm{an}}$, then the complex current $T_{\sigma}$ is the push-forward of a positive current on $U \cap \overline{O(\sigma)}^{\text {an }}$ if and only if for all $\rho \in \Sigma$ and all choices of toric coordinates on $U_{\rho}$ the co-coefficients $T_{\sigma}^{I J}$ are zero whenever $O(\sigma) \subset E^{I \cup J}$.

(b) If $U \subset N_{\Sigma}$, then there is a positive Lagerberg current on $U \cap \overline{N(\sigma)}$ whose image on $U$ is $T_{\sigma}$.

We will see in the proof below that in the Lagerberg case, the necessary and sufficient condition in (a) is always satisfied which is the reason for (b) to be true. 
Proof We first deal with the complex case (a). Suppose that there is a current $S$ on $U$ such that $\iota_{*}(S)=T_{\sigma}$ for the closed immersion $\iota$ of $U \cap \overline{O(\sigma)}$ an into $U$. Let $\rho \in \Sigma$ and let us fix toric coordinates on $U_{\rho}$ such that we may view $U_{\rho}$ as an open subset of $\mathbb{C}^{n}$. Suppose that $N(\sigma) \subset E^{I \cup J}$. This means that there is $k \in I \cup J$ such that the coordinate $z_{k}$ vanishes on $O(\sigma)$. Hence the restrictions of $d z_{k}, d \bar{z}_{k}$ to $O(\sigma)$ are zero. This implies that $T_{\sigma}^{I J}=0$ using the definition of co-coefficients and $\iota_{*}(S)=T_{\sigma}$.

Conversely, assume that for any $\rho \in \Sigma$ and any choice of toric coordinates on $U_{\rho}$, we have $T_{\sigma}^{I J}=0$ on $U_{\rho}$ whenever $N(\sigma) \subset E^{I \cup J}$. The coordinates $z_{k}$ of $O(\sigma)$ are precisely those such that $z_{k} \neq 0$ on $O(\sigma)$. We conclude that the co-coefficients of a current $S$ on $U_{\rho} \cap \overline{O(\sigma)}{ }^{\text {an }}$ can be labeled as $S^{I J}$ with $I, J \subset\{1, \ldots, n\}$ such that $|I|=|J|=p$ and such that $N(\sigma)$ is not contained in $E^{I \cup J}$. To construct such a current $S$ with $\iota_{*}(S)=T_{\sigma}$, we define its co-coefficients on $U_{\rho} \cap \overline{O(\sigma)}^{\text {an }}$ by

$$
S^{I J}(f):=T_{\sigma}^{I J}(g)
$$

where $f$ is any smooth function with compact support in $U_{\rho} \cap \overline{O(\sigma)}^{\text {an }}$ and where $g$ is any extension to a smooth function with compact support in $U_{\rho}$. It follows from Theorem 6.1.6 that the co-coefficients of $T_{\sigma}$ depend only on the restriction to $U_{\rho} \cap \overline{O(\sigma)}^{\text {an }}$. Hence the definition of $S^{I J}$ depends only on $f$ and not on the choice of the extension $g$. By Remark 5.2.4, there is a unique current $S_{\rho}$ on $U_{\rho} \cap \overline{O(\sigma)}^{\text {an }}$ with co-coefficients $S^{I J}$. By definition, $T_{\sigma}^{I J}$ is the push-forward of $S^{I J}$. This shows that $\left.T_{\sigma}\right|_{U_{\rho}}$ is the push-forward of $S_{\rho}$ with respect to the closed immersion of $U_{\rho} \cap \overline{O(\sigma)}^{\text {an }}$ into $U_{\rho}$. It follows that the currents $S_{\rho}$ on the open covering $U_{\rho}$ of $U$ glue to a current $S$ on $U$ with $\iota_{*}(S)=T_{\sigma}$. Since $T_{\sigma}$ is positive and since we can extend compactly supported positive forms on $U \cap \overline{N(\sigma)}$ to compactly supported positive forms $U$, it is clear that also $S$ is positive. This proves (a).

In the case of a positive Lagerberg current on $U$, we note that Theorem 6.1.6 says that the support of $T_{\sigma}$ is contained in $U \cap \overline{N(\sigma)}$. For any $\rho \in \Sigma$ and any choice of toric coordinates on $U_{\rho}$, the co-coefficient $T_{\sigma}^{I J}$ on $U_{\rho} \backslash E^{I \cup J}$ has support contained in $\operatorname{supp}\left(T_{\sigma}\right)$. If $I, J$ satisfy $N(\sigma) \subset E^{I \cup J}$, then the support of the restriction of $T_{\sigma}$ to $U_{\rho}$ is contained in $U_{\rho} \cap \overline{N(\sigma)} \subset$ $E^{I \cup J}$ and hence $T_{\sigma}^{I J}=0$ on $U_{\rho} \backslash E^{I \cup J}$. We conclude that the crucial condition in (a) is always satisfied in the Lagerberg case and an easy adaption of the argument of (a) to the Lagerberg case gives a positive Lagerberg current $S$ on $U \cap N(\sigma)$ whose image in $U$ is $T_{\sigma}$ proving (b).

Lemma 6.1.9 Let $U$ be an open subset of $\mathbb{R}_{\infty}^{n}$ and $V:=\operatorname{trop}^{-1}(U) \subset \mathbb{C}^{n}$. We consider $S \in D^{p, p}(V)^{\mathbb{S}, F}$ and $T:=\operatorname{trop}_{*}(S)$. Let $I, J \subset\{1, \ldots, n\}$ with $|I|=|J|=q:=n-p$.

(i) For any $f \in A_{c}^{0,0}\left(U \backslash E^{I \cup J}\right)$, we have

$$
T^{I J}(f)=\pi^{-q} 2^{-2 q} S^{I J}\left(z^{-I} \bar{z}^{-J} \operatorname{trop}^{*}(f)\right) .
$$

(ii) Assume that the current $S$ is positive. Then $T$ is positive as well and the total variation measures $\left|S^{I J}\right|$ and $\left|T^{I J}\right|$ of the Radon measures $S^{I J}$ and $T^{I J}$ satisfy

$$
\left|S^{I J}\right|\left(\operatorname{trop}^{*}(g)\right)=\pi^{-q} 2^{2 q}\left|T^{I J}\right|\left(\prod_{i \in I} e^{-u_{i}} \prod_{j \in J} e^{-u_{j}} g\right)
$$

for each $g \in A_{c}^{0,0}\left(U \backslash E^{I \cup J}\right)$. 
Proof Recall from Lemma 5.1.5 that $T \in D^{p, p}(U)$. To prove (i), we use (4.5) to get

$$
\begin{aligned}
T^{I J}(f) & =T\left((-1)^{\frac{q(q-1)}{2}} f d^{\prime} u_{I} \wedge d^{\prime \prime} u_{J}\right)=S\left(i^{q^{2}-q} \operatorname{trop}^{*}\left(f d^{\prime} u_{I} \wedge d^{\prime \prime} u_{J}\right)\right) \\
& =S\left(i^{q^{2}} \pi^{-q} \operatorname{trop}^{*}(f) \frac{d z_{I}}{2^{q} z^{I}} \wedge \frac{i^{q} d \bar{z}_{J}}{2^{q} \bar{z}^{J}}\right)=\pi^{-q} 2^{-2 q} S^{I J}\left(z^{-I} \bar{z}^{-J} \operatorname{trop}^{*}(f)\right) .
\end{aligned}
$$

To prove (ii), we recall from Proposition 5.1.13 that $T$ is positive if $S$ is positive. Now equation (6.4) follows from (6.3) by using $\left|z^{I} \bar{z}^{J}\right|=\prod_{i \in I} e^{-u_{i}} \prod_{j \in J} e^{-u_{j}}$.

The following result shows compatibility of the tropicalization map with the canonical decomposition of positive currents from Theorem 6.1.6.

Proposition 6.1.10 Let $U$ be an open subset of $N_{\Sigma}$ and $V:=\operatorname{trop}^{-1}(U)$. If $S=\sum_{\sigma \in \Sigma} S_{\sigma}$ is the canonical decomposition of the positive current $S \in D^{p, p}(V)^{\mathbb{S}, F}$, then $\operatorname{trop}_{*}(S)$ is a positive Lagerberg current on $U$ with canonical decomposition

$$
\operatorname{trop}_{*}(S)=\sum_{\sigma \in \Sigma} \operatorname{trop}_{*}\left(S_{\sigma}\right) .
$$

Proof We first observe that with $S$ each summand $S_{\sigma}$ is again $\mathbb{S}$ - and $F$-invariant by the uniqueness property of the canonical decomposition. To prove (6.5), we may argue locally on the base and so we may assume that $U=U_{\rho}$ and $V=V_{\rho}$ for some $\rho \in \Sigma$. We choose toric coordinates to view $U$ as an open subset of $\mathbb{R}_{\infty}^{n}$ and $V$ as an open subset of $\mathbb{C}^{n}$. We write $T:=\operatorname{trop}_{*}(S)$ and denote by $T=\sum_{\sigma} T_{\sigma}$ its canonical decomposition. We pick $\sigma \in \Sigma$ and we may assume $S=S_{\sigma}$. Then we have to show that $T=T_{\sigma}$. By our characterization of the canonical decomposition in Theorem 6.1.6, it is equivalent to show that $U \backslash\left(N(\sigma) \cup E^{I \cup J}\right.$ ) are null sets with respect to the Radon measures $\left|T^{I J}\right|$. We note that $\operatorname{trop}^{-1}(N(\sigma))=O(\sigma)$ and that $V \backslash O(\sigma)$ is a null set with respect to the Radon measure $S^{I J}$ using $S=S_{\sigma}$ and Theorem 6.1.6. Then (6.4) yields that $U \backslash\left(N(\sigma) \cup E^{I \cup J}\right)$ is a null set with respect to $\left|T^{I J}\right|$ proving (6.5).

\subsection{Positive Lagerberg currents and local mass}

Equation (6.4) gives a necessary condition for a positive Lagerberg current to be the image of an $\mathbb{S}$ - and $F$-invariant positive complex current. In fact, it naturally leads to the following definition which turns out to be also a sufficient condition.

Definition 6.2.1 Let $U$ be an open subset of $N_{\Sigma}$ and let $T \in D^{p, p}(U)$ be a positive Lagerberg current. We say that $T$ has $\mathbb{C}$-finite local mass if for all $\rho \in \Sigma$ and some choice of toric coordinates $u_{1}, \ldots, u_{n}$ on $U_{\rho}$ as in Remark 6.1.2, the corresponding co-coefficients $T^{I J}$, which may be seen as real Radon measures on $U_{\rho} \backslash E^{I \cup J}$ with total variation measures $\left|T^{I J}\right|$, satisfy the condition that the Borel measures given as the image measures

$$
j_{\rho}^{I J}\left(\left|T^{I, J}\right| \prod_{i \in I} e^{-u_{i}} \prod_{j \in J} e^{-u_{j}}\right)
$$

with respect to the open immersion $j_{\rho}^{I J}: U_{\rho} \backslash E^{I \cup J} \rightarrow U_{\rho}$ are locally finite on $U_{\rho}$.

Clearly, the above definition of $\mathbb{C}$-finite local mass does not depend on the choice of toric coordinates on $U_{\rho}$. Note that a Borel measure is locally finite on $U_{\rho}$ if and only if it is a Radon measure on $U_{\rho}$ (see Appendix A). 
Proposition 6.2.2 Let $U \subset N_{\Sigma}$ be an open subset and $V=\operatorname{trop}^{-1}(U)$. Let $T \in D^{p, p}(U)$ be a positive Lagerberg current, then there exists a positive complex current $S \in D^{p, p}(V)^{\mathbb{S}, F}$ such that $\operatorname{trop}_{*}(S)=T$ if and only if $T$ has $\mathbb{C}$-finite local mass.

Proof Suppose that $S \in D^{p, p}(V)^{\mathbb{S}, F}$ is a positive current and let $T=\operatorname{trop}_{*}(S)$. We will prove that $T$ has $\mathbb{C}$-finite local mass. Indeed, it follows from (6.4) that we have the identity

$$
\operatorname{trop}\left(\left|S^{I J}\right|\right)=\pi^{-q} 2^{2 q} \prod_{i \in I} e^{-u_{i}} \prod_{j \in J} e^{-u_{j}}\left|T^{I J}\right|
$$

of positive measures on $U_{\rho} \backslash E^{I \cup J}$ for any $\rho \in \Sigma$ and any choice of toric coordinates $u_{1}, \ldots, u_{n}$ on $U_{\rho}$. Passing to image measures with respect to the open immersion $j_{\rho}^{I J}: U_{\rho} \backslash$ $E^{I \cup J} \rightarrow U_{\rho}$ and using that $\operatorname{trop}\left(\left|S^{I J}\right|\right)$ is a Borel measure on $U_{\rho}$, we deduce that

$$
\operatorname{trop}\left(\left|S^{I J}\right|\right) \geq \pi^{-q} 2^{2 q} j_{\rho}^{I J}\left(\prod_{i \in I} e^{-u_{i}} \prod_{j \in J} e^{-u_{j}}\left|T^{I J}\right|\right) \geq 0 .
$$

By Proposition 5.2.5, the Borel measure $\left|S^{I J}\right|$ is locally finite on $V_{\rho}$ and hence the Borel measure $\operatorname{trop}\left(\left|S^{I J}\right|\right)$ is locally finite on $U_{\rho}$. We deduce that $T$ has $\mathbb{C}$-finite local mass.

Conversely, let $T$ be a positive Lagerberg current in $D^{p, p}(U)$ with $\mathbb{C}$-finite local mass. We consider the canonical decomposition $T=\sum_{\sigma \in \Sigma} T_{\sigma}$ from Theorem 6.1.6. First, we assume that $T=T_{\{0\}}$ for the minimal cone $\{0\} \in \Sigma$. By assumption, the co-coefficients are Radon measures $T^{I J}$ on $U_{\rho} \backslash E^{I \cup J}$ such that the positive Borel measures

$$
j_{\rho}^{I J}\left(\left|T^{I J}\right| \prod_{i \in I} e^{-u_{i}} \prod_{j \in J} e^{-u_{j}}\right)
$$

are locally finite on $U_{\rho}$ for every $\rho \in \Sigma$. By Remark 5.1.6, there is a unique current $R \in$ $D^{p, p}\left(V \cap \mathbb{T}^{\text {an }}\right)^{\mathbb{S}, F}$ with $\operatorname{trop}_{*}(R)=\left.T\right|_{N_{\mathbb{R}}}$. Since $T$ is positive, it follows from Lemma 5.1.14 that $R$ is a positive current. It follows from Proposition 5.2.5 that the co-coefficients $R^{I J}$ of $R$ are complex Radon measures on $V \cap \mathbb{T}^{\text {an }}$. Using (6.4), we get the following identity of Borel measures

$$
\operatorname{trop}\left(\left|R^{I J}\right|\right)=\pi^{-q} 2^{2 q} \prod_{i \in I} e^{-u_{i}} \prod_{j \in J} e^{-u_{j}}\left|T^{I J}\right|
$$

on $U \cap N_{\mathbb{R}}$. Using that $T$ has $\mathbb{C}$-finite local mass, we know that the image measure of the right hand side with respect to the open immersion $j_{\rho}: U \cap N_{\mathbb{R}} \rightarrow U_{\rho}$ is a locally finite Borel measure on $U_{\rho}$. We conclude that the image measure of $\left|R^{I J}\right|$ with respect to the open immersion $j_{\rho}^{\prime}: V \cap \mathbb{T}^{\text {an }} \rightarrow V_{\rho}$ is a locally finite Borel measure on $V_{\rho}$ by using $j_{\sigma} \circ$ trop $=\operatorname{trop} \circ j_{\sigma}^{\prime}$ on $V \cap \mathbb{T}^{\text {an }}$. Hence the Radon measure $R^{I J}$ admits an image Radon measure $j_{\rho}^{\prime}\left(R^{I J}\right)$ which is a complex Radon measure on $V_{\rho}$. By Remark 5.2.4 and Example 3.2.11, there is a unique current in $S \in D^{p, p}\left(V_{\rho}\right)$ with co-coefficients $S^{I J}$ induced by $j_{\rho}^{\prime}\left(R^{I J}\right)$. Since the restriction of the co-coefficients $S^{I J}$ to the dense stratum $V \cap \mathbb{T}^{\text {an }}$ is $R^{I J}$, it follows also from Remark 5.2.4 that $\left.S\right|_{V \cap \mathbb{T}^{\text {an }}}=R$. By construction, the boundary $V_{\rho} \backslash N_{\mathbb{R}}$ is a null set with respect to each Radon measure $j_{\rho}^{\prime}\left(R^{I J}\right)$. By Lemma 6.1.4 and Remark 6.1.5, there is an increasing sequence $\psi_{k} \geq 0$ in $A_{c}^{0,0}\left(V \backslash N_{\mathbb{R}}\right)^{\mathbb{S}}$ which converges pointwise to the characteristic function of $V \backslash N_{\mathbb{R}}$ such that for any $\alpha \in A_{c}^{q, q}\left(V_{\rho}\right)$ we have

$$
\lim _{k \rightarrow \infty} S(\alpha)=\lim _{k \rightarrow \infty} S\left(\psi_{k} \alpha\right)=\lim _{k \rightarrow \infty} R\left(\psi_{k} \alpha\right) .
$$


It follows that the currents $S$, defined a priori only on $V_{\rho}$ for any $\rho \in \Sigma$, do not depend on the choice of toric coordinates on $V_{\rho}$ and hence agree on overlappings of the covering $\left(V_{\rho}\right)_{\rho \in \Sigma}$. They define a current on $V$ which we also denote by $S$. If $\alpha$ is positive, then $\psi_{k} \alpha$ is also positive. By positivity of $R$ and by (6.6), the current $S$ is also positive. Since both $\psi_{k}$ and $R$ are invariant with respect to $\mathbb{S}$ and $F$, we deduce from (6.6) that $S \in D^{p, p}(V)^{\mathbb{S}, F}$.

We have to check $\operatorname{trop}_{*}(S)=T$. Using $j_{\sigma} \circ$ trop $=\operatorname{trop} \circ j_{\sigma}^{\prime}$ on $V \cap \mathbb{T}^{\text {an }}$, we have the following identities

$$
\begin{aligned}
\operatorname{trop}\left(\pi^{-q} 2^{-2 q} z^{-I} \bar{z}^{-J} S^{I J}\right) & =\operatorname{trop}\left(j_{\sigma}^{\prime}\left(\pi^{-q} 2^{-2 q} z^{-I} \bar{z}^{-J} R^{I J}\right)\right) \\
& =j_{\sigma}\left(\operatorname{trop}\left(\pi^{-q} 2^{-2 q} z^{-I} \bar{z}^{-J} R^{I J}\right)\right)
\end{aligned}
$$

of Radon measures on $U_{\rho}$. By (6.3), we deduce the identity of Radon measures

$$
\left(\operatorname{trop}_{*}(S)\right)^{I J}=\operatorname{trop}\left(\pi^{-q} 2^{-2 q} z^{-I} \bar{z}^{-J} S^{I J}\right)=j_{\sigma}\left(\operatorname{trop}\left(\pi^{-q} 2^{-2 q} z^{-I} \bar{z}^{-J} R^{I J}\right)\right)
$$

on $U_{\rho} \backslash E^{I \cup J}$. Since $\operatorname{trop}_{*}(R)=T$ on $U \cap N_{\mathbb{R}}$, we deduce again from (6.3)

$$
\left(\operatorname{trop}_{*}(S)\right)^{I J}=j_{\sigma}\left(\operatorname{trop}\left(2^{-2 q} z^{-I} \bar{z}^{-J} R^{I J}\right)\right)=T^{I J}
$$

on $U_{\rho} \backslash E^{I \cup J}$, where in the last step we have used our assumption $T=T_{\{0\}}$. Then Remark 5.2.4 proves that $\operatorname{trop}_{*}(S)=T$.

Now we skip the assumption $T=T_{\{0\}}$ from above and consider any positive current $T$ with canonical decomposition $T=\sum_{\sigma \in \Sigma} T_{\sigma}$ from Theorem 6.1.6. By Proposition 6.1.8, for every $\sigma \in \Sigma$, there is a positive Lagerberg current $P_{\sigma}$ on $U \cap \overline{N(\sigma)}$ such that $T_{\sigma}=\left(\iota_{\sigma}\right)_{*}\left(P_{\sigma}\right)$ for the closed immersion $\iota_{\sigma}: U \cap \overline{N(\sigma)} \rightarrow U$. Now we apply the above case to the current $P_{\sigma}$ and to the open subset $V \cap \overline{O(\sigma)}$ an of the toric variety $\overline{O(\sigma)}$. We conclude that there is a positive current $Q_{\sigma}$ on $V \cap \overline{O(\sigma)}$ an such that $\operatorname{trop}_{*}\left(Q_{\sigma}\right)=P_{\sigma}$. For the closed immersion $\iota_{\sigma}^{\prime}: V \cap \overline{O(\sigma)}^{\text {an }} \rightarrow V$, we have the obvious relation trop $\circ \iota_{\sigma}^{\prime}=\iota_{\sigma} \circ$ trop. Now we set $S_{\sigma}:=\left(\iota_{\sigma}^{\prime}\right)_{*}\left(Q_{\sigma}\right)$ which is a positive current on $V$. Then we get

$$
\operatorname{trop}_{*}\left(S_{\sigma}\right)=\operatorname{trop}_{*}\left(\left(\iota_{\sigma}^{\prime}\right)_{*}\left(Q_{\sigma}\right)\right)=\left(\iota_{\sigma}\right)_{*}\left(\operatorname{trop}_{*}\left(Q_{\sigma}\right)\right)=\left(\iota_{\sigma}\right)_{*}\left(P_{\sigma}\right)=T_{\sigma} .
$$

Then $S:=\sum_{\sigma \in \Sigma} S_{\sigma}$ is a positive current on $V$ satisfying $\operatorname{trop}_{*}(S)=T$.

Next we give an example of a positive Lagerberg current $T$ such that $T=\operatorname{trop}_{*}(S)$ for some complex current $S$, but for which no such $S$ is positive.

Example 6.2.3 We consider the one-dimensional situation with $N=\mathbb{Z}, N_{\Sigma}=\mathbb{R}_{\infty}$ and $X_{\Sigma}=\mathbb{A}_{\mathbb{C}}^{1}$. Write $U \subset N_{\Sigma}$ given by $U=\left\{u \in \mathbb{R}_{\infty} \mid u>0\right\}$ and $V=\operatorname{trop}^{-1}(U)=\{z \in$ $\mathbb{C} \mid z \bar{z}<1\}$. Let $T \in D^{0,0}(U)$ be the current

$$
T\left(g d^{\prime} u \wedge d^{\prime \prime} u\right)=\int_{0}^{\infty} g(x) e^{2 x} d x .
$$

Since the function $1=e^{2 x} e^{-2 x}$ does not have locally finite mass around $\infty$, this current is not of the form $\operatorname{trop}_{*}(S)$ for a positive current $S$ in $V$. Nevertheless $T$ is in the image of trop . Indeed, let $S \in D^{0,0}(U)$ be the current given by

$$
S(f d z \wedge d \bar{z})=\int_{V} \frac{f(z)-f(0)}{z \bar{z}} d z \wedge d \bar{z} .
$$


Clearly this current is not weakly positive. Let now $g d^{\prime} u \wedge d^{\prime \prime} u$ be a Lagerberg form on $U$. Then $g(u)=0$ for $u \gg 0$ and hence

$$
\begin{aligned}
\operatorname{trop}_{*} S\left(g d^{\prime} u \wedge d^{\prime \prime} u\right) & =S\left(\operatorname{trop}^{*}\left(g d^{\prime} u \wedge d^{\prime \prime} u\right)\right)=S\left(g(-\log |z|) \frac{i d z \wedge d \bar{z}}{4 \pi z \bar{z}}\right) \\
& =\frac{1}{\pi} \int_{V} \frac{g(-\log |z|)}{4(z \bar{z})^{2}} i d z \wedge d \bar{z}=\int_{0}^{1} g(-\log r) \frac{r d r}{r^{4}}=\int_{1}^{\infty} g(x) e^{2 x} d x .
\end{aligned}
$$

This shows $T=\operatorname{trop}_{*} S$.

\section{The correspondence theorem for closed positive currents}

In this section, we consider a smooth fan $\Sigma$ with associated toric variety $X_{\Sigma}$ and partial compactification $N_{\Sigma}$. After considering positivity of currents in the previous sections, we add here the additional condition that the currents are closed. Recall that a complex $(p, p)$ current $T$ (resp. a Lagerberg $(p, p)$-current $S)$ is called closed if $\partial T=\bar{\partial} T=0\left(\right.$ resp. $d^{\prime} S=$ $d^{\prime \prime} S=0$ ). In the first subsection, we show our main theorem. It states that the tropicalization map induces a bijective correspondence between $\mathbb{S}$ - and $F$-invariant closed positive currents on $X_{\Sigma}$ and closed positive Lagerberg currents on $N_{\Sigma}$. In the second subsection, we derive from our main theorem a tropical version of the Skoda-El Mir theorem.

\subsection{Closed positive invariant currents}

For $M \subset M^{\prime} \subset\{1, \ldots, n\}$, we consider the inclusions $\mathbb{R}_{\infty}^{M} \subset \mathbb{R}_{\infty}^{M^{\prime}} \subset \mathbb{R}_{\infty}^{n}$ obtained by adding zero at the missing components. For $v \in \mathbb{R}_{\geq 0}^{M}$, we consider the parallelepiped

$$
P(v):=\left\{u \in \mathbb{R}^{M} \mid 0 \leq u_{i} \leq v_{i} \forall i \in M\right\}
$$

Lemma 7.1.1 Let $T$ be a closed current in $D_{+}^{p, p}(U)$ for an open subset $U$ of $\mathbb{R}_{\infty}^{n}$. Fix $M \subset$ $\{1, \ldots, n\}$ with $|M|=n-p$ and with complement $M^{c}$. Fix also a compact subset $K$ of $U$, and a function $\chi \in C_{c}^{\infty}\left(\mathbb{R}_{\infty}^{M^{c}}\right)$. Then there is a constant $C \in \mathbb{R}_{\geq 0}$ such that for all $f \in C_{c}^{\infty}\left(\mathbb{R}^{M}\right)$, and for all $v \in \mathbb{R}_{\geq 0}^{M}$ such that $(\operatorname{supp}(f)+P(v)) \times \operatorname{supp}(\bar{\chi}) \subset K$, we have

$$
\left|T\left(f\left(u_{M}\right) \chi\left(u_{M^{c}}\right) d^{\prime} u_{M} \wedge d^{\prime \prime} u_{M}\right)-T\left(f\left(u_{M}-v\right) \chi\left(u_{M^{c}}\right) d^{\prime} u_{M} \wedge d^{\prime \prime} u_{M}\right)\right| \leq C\|v\| \cdot\|f\|_{\text {sup }}
$$

Proof Let $\left(e_{1}, \ldots, e_{n}\right)$ denote the standard basis of $\mathbb{R}^{n}$ and write $v=\sum_{i \in M} t_{i} e_{i}$. Using a telescope argument, one easily reduces to the case where $v=t e_{i}$ for some fixed $i \in M$. For a given $t \in\left[0, v_{i}\right]$, we consider the auxiliary function

$$
g_{t}: \mathbb{R}^{M} \longrightarrow \mathbb{R}, g_{t}\left(u_{M}\right)=\int_{-\infty}^{0} f\left(u_{M}+s e_{i}\right)-f\left(u_{M}+(s-t) e_{i}\right) d s=\int_{-t}^{0} f\left(u_{M}+s e_{i}\right) d s .
$$

From $\left(\operatorname{supp}(f)+P\left(t e_{i}\right)\right) \times \operatorname{supp}(\chi) \subset K$, we get

$$
\operatorname{supp}\left(g_{t}\right) \times \operatorname{supp}(\chi) \subset K, \quad\left\|g_{t}\right\|_{\text {sup }} \leq t\|f\|_{\text {sup }}, \quad \frac{\partial g_{t}}{\partial u_{i}}(u)=f\left(u_{M}\right)-f\left(u_{M}-t e_{i}\right)(7.1)
$$


We put $M^{\prime}=M \backslash\{i\}$ and obtain

$$
\begin{aligned}
d^{\prime}\left(g_{t}\left(u_{M}\right) \chi\left(u_{M^{c}}\right) d^{\prime} u_{M^{\prime}} \wedge d^{\prime \prime} u_{M}\right)= & \sum_{j \in M^{c}} g_{t}\left(u_{M}\right) \frac{\partial \chi}{\partial u_{j}}\left(u_{M^{c}}\right) d^{\prime} u_{j} \wedge d^{\prime} u_{M^{\prime}} \wedge d^{\prime \prime} u_{M} \\
& +\left(f\left(u_{M}\right)-f\left(u_{M}-t e_{i}\right)\right) \chi\left(u_{M^{c}}\right) d^{\prime} u_{i} \wedge d^{\prime} u_{M^{\prime}} \wedge d^{\prime \prime} u_{M} .
\end{aligned}
$$

Since $g_{t}$ has compact support in $\mathbb{R}^{M}$ and, by (7.1) $g_{t}\left(u_{M}\right) \chi\left(u_{M^{c}}\right)$ has compact support in $U$, we deduce that

$$
g_{t}\left(u_{M}\right) \chi\left(u_{M^{c}}\right) d^{\prime} u_{M^{\prime}} \wedge d^{\prime \prime} u_{M} \in A_{c}^{n-p-1, n-p}(U) .
$$

Hence, since $T$ is closed, we have

$$
T\left(d^{\prime}\left(g_{t}\left(u_{M}\right) \chi\left(u_{M^{c}}\right) d^{\prime} u_{M^{\prime}} \wedge d^{\prime \prime} u_{M}\right)\right)=0 .
$$

Therefore, using that $T$ has measure co-coefficients (see Proposition 5.2.6), we get

$$
\begin{aligned}
& \left|T\left(\left(f\left(u_{M}\right)-f\left(u_{M}-t e_{i}\right)\right) \chi\left(u_{M^{c}}\right) d^{\prime} u_{M} \wedge d^{\prime \prime} u_{M}\right)\right| \\
& \quad \leq \sum_{j \in M^{c}}\left|T^{M^{\prime} \cup\{j\}, M}\right|\left(\left|\frac{\partial \chi}{\partial u_{j}}\left(u_{M^{c}}\right) g_{t}\left(u_{M}\right)\right|\right) \\
& \quad \leq \sum_{j \in M^{c}}\left|T^{M^{\prime} \cup\{j\}, M}\right|\left(\left|\frac{\partial \chi}{\partial u_{j}}\left(u_{M^{c}}\right)\right|\right)\left\|g_{t}\right\|_{\text {sup }} \\
& \quad \leq C \cdot t \cdot\|f\|_{\text {sup }}
\end{aligned}
$$

for some constant $C$.

Proposition 7.1.2 Let $U \subset N_{\Sigma}$ be an open subset and $T \in D^{p, p}(U)$. If $T$ is positive and closed, then it has $\mathbb{C}$-finite local mass.

Proof The question is local and so we may assume that $U$ is an open subset of $\mathbb{R}_{\infty}^{n}$.

If $p=n$, then the only co-coefficient of $T$ is $T^{\emptyset, \emptyset}$ which is a positive Radon measure on all of $U$ by Proposition 5.2.6. Hence $T$ has $\mathbb{C}$-finite local mass.

Now assume that $p<n$. We want to prove that $T$ has $\mathbb{C}$-finite local mass around $u \in U$. We will check the relevant condition in Definition 6.2.1 first for the co-coefficients $T^{M M}$. For notational simplicity, we assume $M=\{1, \ldots, n-p\}$ and $u=(\infty, \ldots, \infty)$ which is no real restriction of generality as finite components are easier to handle. We choose a compact neighborhood $K$ of $u$. There is $R \in \mathbb{N}_{\geq 1}$ such that $[R-1, \infty]^{n} \subset K$. There is a $C^{\infty}$-function $f$ on $\mathbb{R}^{M}$ with the properties:

(i) $0 \leq f \leq 1$;

(ii) $f \equiv 1$ on the unit cube $[R, R+1]^{M}$;

(iii) $f$ has compact support in the open cube $\left(R-\frac{1}{2}, R+\frac{3}{2}\right)^{M}$.

We choose $\chi \in C^{\infty}\left(\mathbb{R}_{\infty}^{M^{c}}\right)$ with compact support in $\left(R-\frac{1}{2}, \infty\right]^{M^{c}}$ such that $0 \leq \chi \leq 1$ and $\chi \equiv 1$ on $[R, \infty]^{M^{c}}$. For $\underline{n} \in \mathbb{N}^{M}$, we consider the positive Lagerberg form

$$
\alpha_{M, \underline{n}}=(-1)^{\frac{(n-p)(n-p-1)}{2}} \chi\left(u_{M^{c}}\right) f\left(u_{M}-\underline{n}\right) d^{\prime} u_{M} \wedge d^{\prime \prime} u_{M} .
$$

Lemma 7.1.1 implies that

$$
T\left(\alpha_{M, \underline{n}}\right)=T\left(\alpha_{M, \underline{0}}\right)+O(\|\underline{n}\|) .
$$


For any $\varepsilon>0$, we prove now that the positive measure

$$
T^{M M} \prod_{i \in M} \frac{1}{u_{i}^{2+2 \varepsilon}}
$$

has finite mass in the region $S:=[R, \infty)^{M} \times[R, \infty]^{M^{c}}$. We have

$$
\begin{aligned}
\left(T^{M M} \prod_{i \in M} \frac{1}{u_{i}^{2+2 \varepsilon}}\right)\left(1_{S}\right) & \leq \sum_{\underline{n} \in\left(\mathbb{N}_{\geq 1}\right)^{M}}\left(\prod_{i \in M} \frac{1}{n_{i}^{2+2 \varepsilon}}\right) T\left(\alpha_{M, \underline{n}}\right) \\
& =\sum_{\underline{n} \in\left(\mathbb{N}_{\geq 1}\right)^{M}}\left(\prod_{i \in M} \frac{1}{n_{i}^{2+2 \varepsilon}}\right) O(\|\underline{n}\|) \\
& \leq O(1) \sum_{\underline{n} \in\left(\mathbb{N}_{\geq 1}\right)^{M}} \prod_{i \in M} \frac{1}{n_{i}^{1+2 \varepsilon}}<\infty .
\end{aligned}
$$

For $I, J \subset\{1, \ldots, n\}$ with $|I|=|J|=n-p$, Eq. (5.8) yields

$$
\left|T^{I J}\right| \prod_{i \in I} \frac{1}{u_{i}^{1+\varepsilon}} \prod_{j \in J} \frac{1}{u_{j}^{1+\varepsilon}} \leq \frac{1}{2}\left(T^{I I} \prod_{i \in I} \frac{1}{u_{i}^{2+2 \varepsilon}}+T^{J J} \prod_{j \in J} \frac{1}{u_{j}^{2+2 \varepsilon}}\right)
$$

in the region $[R, \infty)^{I \cup J} \times[R, \infty]^{(I \cup J)^{c}}$. Therefore the left hand side has finite mass in this region. Finally, using that $e^{-u}$ decreases faster than $u^{-1-\varepsilon}$, we deduce that $T$ has $\mathbb{C}$-finite local mass.

Proposition 7.1.3 Let $U \subset N_{\Sigma}$ be an open subset and $V=\operatorname{trop}^{-1}(U)$. Let $T^{\prime}, T^{\prime \prime} \in$ $D^{p, p}(V)^{\mathbb{S}, F}$ be positive and closed currents. If $\operatorname{trop}_{*}\left(T^{\prime}\right)=\operatorname{trop}_{*}\left(T^{\prime \prime}\right)$, then $T^{\prime}=T^{\prime \prime}$.

Proof This can be proved locally on $N_{\Sigma}$. Hence we may assume that $\Sigma$ is a fan containing a single maximal dimensional cone with all its faces, so that $N_{\Sigma}=\mathbb{R}_{\infty}^{n}$ as in Definition 6.1.2. Then the cones of $\Sigma$ are given by the faces

$$
\sigma_{M}:=\left\{u \in \mathbb{R}_{\geq 0}^{n} \mid u_{m}=0 \forall m \notin M\right\}
$$

of $\mathbb{R}_{+}^{n}$, where $M$ is ranging over all subsets of $\{1, \ldots, n\}$. We have the corresponding strata

$$
S_{M}:=O\left(\sigma_{M}\right)^{\text {an }} \cap V=\left\{\left(z_{1}, \ldots, z_{n}\right) \in V \mid z_{i}=0 \Leftrightarrow i \in M\right\}
$$

of $V$. We consider now the canonical decompositions of $T^{\prime}$ and $T^{\prime \prime}$ from Theorem 6.1.6:

$$
T^{\prime}=\sum_{\sigma \in \Sigma} T_{\sigma}^{\prime}, \quad T^{\prime \prime}=\sum_{\sigma \in \Sigma} T_{\sigma}^{\prime \prime} .
$$

For $M \subset\{1, \ldots, n\}$ and $\sigma:=\sigma_{M} \in \Sigma$, we recall that $T_{\sigma}^{\prime}$ and $T_{\sigma}^{\prime \prime}$ are $\mathbb{S}$-invariant, positive currents with support contained in the closure $\overline{S_{M}}$ of the stratum $S_{M}$. The co-coefficients of the current $T_{\sigma}:=T_{\sigma}^{\prime}-T_{\sigma}^{\prime \prime}$ are obtained by first restricting the co-coefficient measures of $T:=T^{\prime}-T^{\prime \prime}$ to $S_{M}$ and then by extending these measures by zero to $V$.

We claim that all currents $T_{\sigma}, T_{\sigma}^{\prime}$ and $T_{\sigma}^{\prime \prime}$ are closed. It is clear that $T^{\prime}$ agrees with $T_{\{0\}}^{\prime}$ on the dense stratum $V \cap \mathbb{T}^{\text {an }}$ and hence $\left.T_{\{0\}}^{\prime}\right|_{V \cap \mathbb{T}^{\text {an }}}$ is a closed positive current on $V \cap \mathbb{T}^{\text {an }}$. Since $T_{\{0\}}^{\prime}$ is a positive current on $V$, it is clear that the restriction of $T_{\{0\}}^{\prime}$ to $V \cap T^{\text {an }}$ has locally finite mass near the boundary $\partial V:=V \backslash\left(V \cap \mathbb{T}^{\mathrm{an}}\right)$. By the Skoda-El Mir Theorem [9, Theorem III.2.3] and using that $\partial V$ is a null set for $T_{\{0\}}^{\prime}$, it follows that $T_{\{0\}}^{\prime}$ is closed on 
$V$. Using induction on $\operatorname{dim}(\sigma)$, we can prove similarly that $T_{\sigma}^{\prime}$ is closed for any $\sigma \in \Sigma$. The same arguments show that $T_{\sigma}^{\prime \prime}$ is closed. Then $T_{\sigma}$ is closed as well.

From Proposition 6.1.10, we get the equality of canonical decompositions

$$
\sum_{\sigma \in \Sigma} \operatorname{trop}_{*}\left(T_{\sigma}^{\prime}\right)=\operatorname{trop}_{*}\left(T^{\prime}\right)=\operatorname{trop}_{*}\left(T^{\prime \prime}\right)=\sum_{\sigma \in \Sigma} \operatorname{trop}_{*}\left(T_{\sigma}^{\prime \prime}\right)
$$

which implies $\operatorname{trop}_{*}\left(T_{\sigma}\right)=0$ for all $\sigma \in \Sigma$.

To prove the proposition, it is enough to show for each $\sigma=\sigma_{M} \in \Sigma$ that $T_{\sigma}$ is zero, or equivalently that all co-coefficients $T_{\sigma}^{I J}$ are zero for all subsets $I, J \subset\{1, \ldots, n\}$ with $|I|=|J|=n-p$ (see Remark 5.2.4). We prove this by induction on the cardinality of $M \cap(I \cup J)$. For the initial step, we consider subsets $M, I, J \subset\{1, \ldots, n\}$ such that $M \cap(I \cup J)=\emptyset$. Let $f$ be a real valued smooth function on $U$ with compact support in $U \backslash E^{I \cup J}=\left\{u \in U \mid u_{i} \neq \infty \forall i \in I \cup J\right\}$. Using $\operatorname{trop}_{*}\left(T_{\sigma}\right)=0$ and (6.3), we obtain

$$
T_{\sigma}^{I J}\left(z^{-I} \bar{z}^{-J} \operatorname{trop}^{*}(f)\right)=0 .
$$

Using that $T_{\sigma}^{I J}$ is a Radon measure, we conclude by a standard approximation argument that (7.2) holds for all $f \in C_{c}^{0}\left(U \backslash E^{I \cup J}\right)$.

For any $g \in C_{c}^{0}\left(V \backslash \operatorname{trop}^{-1}\left(E^{I \cup J}\right)\right)$, let $g^{\text {av }}$ be the natural projection onto the $\mathbb{S}$-invariant functions given by averaging over the fibers of trop with respect to the probability Haar measures. By construction, there is a unique $f \in C_{c}^{0}\left(U \backslash E^{I \cup J}\right)$ with $\operatorname{trop}^{*}(f)=g^{\text {av }}$. The S-invariance of $T_{\sigma}$ implies that

$$
T_{\sigma}^{I J}\left(z^{-I} \bar{z}^{-J} g\right)=T_{\sigma}^{I J}\left(z^{-I} \bar{z}^{-J} g^{\mathrm{av}}\right)=T_{\sigma}^{I J}\left(z^{-I} \bar{z}^{-J} \operatorname{trop}^{*}(f)\right)=0 .
$$

This means that the restriction of $T_{\sigma}^{I J}$ to the open subset $V \backslash \operatorname{trop}^{-1}\left(E^{I \cup J}\right)$ is identically zero. Since $V \backslash S_{M}$ is a null set with respect to the Radon measure $T_{\sigma}^{I J}$ and since our assumption $M \cap(I \cup J)=\emptyset$ yields $S_{M} \subset V \backslash \operatorname{trop}^{-1}\left(E^{I \cup J}\right)$, we deduce that $T_{\sigma}^{I J}=0$.

For the inductive step, the induction hypothesis is that $T_{\rho}^{I J}=0$ whenever $\rho=\sigma_{L} \in \Sigma$ with $|L \cap(I \cup J)|<k$ for some $k \geq 1$. Let $M, I, J \subset\{1, \ldots, n\}$ with $|M \cap(I \cup J)|=k$ and $m \in M \cap(I \cup J)$. We have to show that $T_{\sigma}^{I J}(f)=0$ for $\sigma=\sigma_{M}$ and any $f \in C_{c}^{\infty}(V)$. Using that $V \backslash S_{M}$ is a null set with respect to the Radon measure $T_{\sigma}^{I J}$, we may assume that, in a neighborhood of $\overline{S_{M}}$, the function $f$ depends only the variables $z_{j}, j \notin M$. By symmetry, we may also assume without loss of generality that $m \in I$. Write $I^{\prime}=I \backslash\{m\}$. Let $g \in C_{c}^{\infty}(V)$ be the function given by $g=z_{m} f$. By the assumptions on $f$, there is a neighborhood of $\overline{S_{M}}$ where

$$
\frac{\partial g}{\partial z_{m}}=f
$$

and in this neighborhood, $g$ depends only on the variables $z_{j}, j \notin M$, and $z_{m}$. Consider the smooth form $\eta=g d z_{I^{\prime}} \wedge d \bar{z}_{J}$ on $V$ which has compact support. Since $T_{\sigma}$ is closed and has support on $\overline{S_{M}}$, the assumptions on $g$ lead to

$$
0=T_{\sigma}(\partial \eta)=T_{\sigma}\left( \pm f d z_{I} \wedge d \bar{z}_{J}\right)+\sum_{j \notin M \cup I} T_{\sigma}\left(\frac{\partial g}{\partial z_{j}} d z_{j} \wedge d z_{I^{\prime}} \wedge d \bar{z}_{J}\right),
$$

where the sign depends on the position of $m$ in $I$. Let us first consider the case where $m \notin J$. For each $j \notin M \cup I$, we get $\left|M \cap\left(\left(I^{\prime} \cup\{j\}\right) \cup J\right)\right|<k$. Hence the induction hypothesis gives

$$
T_{\sigma}^{\left(I^{\prime} \cup\{j\}\right) J}\left(\frac{\partial g}{\partial z_{j}}\right)=0
$$


and (7.3) implies $T_{\sigma}\left(f d z_{I} \wedge d \bar{z}_{J}\right)=0$ and

$$
T_{\sigma}^{I J}(f)=i^{q^{2}} T_{\sigma}\left(f d z_{I} \wedge d \bar{z}_{J}\right)=0 .
$$

Now consider the case where $m \in J$. We put $J^{\prime}:=J \backslash\{m\}$. For each $j \notin M \cup I$, we write

$$
h_{j}=\bar{z}_{m} \frac{\partial g}{\partial z_{j}} \in C_{c}^{\infty}(V) .
$$

This function depends only on the variables $\left(z_{j^{\prime}}\right)_{j^{\prime} \notin M}$ and $z_{m}$ and, in a neighborhood of $\overline{S_{M}}$, satisfies

$$
\frac{\partial h_{j}}{\partial \bar{z}_{m}}=\frac{\partial g}{\partial z_{j}} .
$$

For the compactly supported smooth form $\alpha_{j}=h_{j} d z_{j} \wedge d z_{I^{\prime}} \wedge d \bar{z}_{J^{\prime}}$ on $V$, we deduce as above that

$$
\begin{aligned}
0 & =T_{\sigma}\left(\bar{\partial} \alpha_{j}\right) \\
& =T_{\sigma}\left( \pm \frac{\partial g}{\partial z_{j}} d z_{j} \wedge d z_{I^{\prime}} \wedge d \bar{z}_{J}\right)+\sum_{j^{\prime} \notin M \cup J} T_{\sigma}\left(\frac{\partial h_{j}}{\partial \bar{z}_{j^{\prime}}} d \bar{z}_{j^{\prime}} \wedge d z_{j} \wedge d z_{I^{\prime}} \wedge d \bar{z}_{J^{\prime}}\right) .
\end{aligned}
$$

For $j \notin M \cup I$ and $j^{\prime} \notin M \cup J$, we get $\mid M \cap\left(\left(I^{\prime} \cup\{j\}\right) \cup\left(J \cup\left\{j^{\prime}\right\}\right) \mid<k\right.$. Hence our induction hypothesis gives

$$
T_{\sigma}\left(\frac{\partial h_{j}}{\partial \bar{z}_{j^{\prime}}} d \bar{z}_{j^{\prime}} \wedge d z_{j} \wedge d z_{I^{\prime}} \wedge d \bar{z}_{J^{\prime}}\right)=0 .
$$

This implies

$$
T_{\sigma}\left(\frac{\partial g}{\partial z_{j}} d \bar{z}_{j} \wedge d z_{I^{\prime}} \wedge d \bar{z}_{J}\right)=0 .
$$

for all $j \notin M \cup I$ which implies $T_{\sigma}^{I J}=0$ by (7.3) as before. This completes the induction and proves the result.

In general the map trop ${ }_{*}: D_{+}^{p, p}(V) \cap D^{p, p}(V)^{\mathbb{S}, F} \rightarrow D_{+}^{p, p}(U)$ is not injective as the following example shows.

Example 7.1.4 Consider $\mathbb{P}_{\mathbb{C}}^{1}$ as a toric variety. Then the $(0,0)$ current that sends the form $f(z) d z \wedge i d \bar{z}$ to the value $f(0)$ is a non-zero positive invariant current, but its image by trop ${ }_{*}$ is zero. Thus, in Proposition 7.1.3 the closedness condition is necessary.

Theorem 7.1.5 Let $U \subset N_{\Sigma}$ be an open subset, $V=\operatorname{trop}^{-1}(U)$. Denote by $D^{p, p}(U)_{\mathrm{cl},+}$ the cone of positive closed currents on $U$ and by $D^{p, p}(V)_{\mathrm{cl},+}^{\mathbb{S}, F}$ the cone of $\mathbb{S}$ - and $F$-invariant

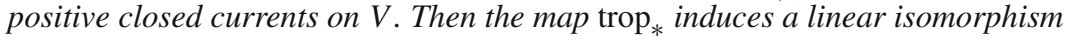

$$
\operatorname{trop}_{*}: D^{p, p}(V)_{\mathrm{cl},+}^{\mathbb{S}, F} \stackrel{\sim}{\longrightarrow} D^{p, p}(U)_{\mathrm{cl},+}
$$

of real cones.

Proof By Lemma 5.1.5, Proposition 4.2.3 and Proposition 5.1.13, the linear map in (7.4) is well-defined. Surjectivity follows from Propositions 6.2.2 and 7.1.2, while injectivity was proven in Proposition 7.1.3. 
The isomorphism in Theorem 7.1.5 respects the support of currents.

Proposition 7.1.6 For $T \in D^{p, p}(V)_{\mathrm{cl},+}^{\mathbb{S}, F}$, we have

$$
\operatorname{trop}^{-1}\left(\operatorname{supp}\left(\operatorname{trop}_{*} T\right)\right)=\operatorname{supp}(T) \text {. }
$$

Proof Recall that the support of a current $T$ is defined as the complement of the maximal open set $W$ such that $\left.T\right|_{W}=0$. Let $C:=\operatorname{supp}\left(\operatorname{trop}_{*}(T)\right)$. Write $U^{\prime}=U \backslash C$ and $V^{\prime}=\operatorname{trop}^{-1}\left(U^{\prime}\right)$. Then $\left.\operatorname{trop}_{*}(T)\right|_{U^{\prime}}=0$ and hence Proposition 7.1.3 yields $\left.T\right|_{V^{\prime}}=0$ which means

$$
\operatorname{supp}(T) \subset \operatorname{trop}^{-1}\left(\operatorname{supp}\left(\operatorname{trop}_{*}(T)\right)\right) .
$$

Since $T$ is $\mathbb{S}$-invariant, then $C^{\prime}:=\operatorname{supp}(T)$ is also $\mathbb{S}$-invariant. Write $V^{\prime \prime}=V \backslash C^{\prime}$. By the invariance of $C^{\prime}$, we have $V^{\prime \prime}=\operatorname{trop}^{-1}\left(U^{\prime \prime}\right)$ for an open subset $U^{\prime \prime} \subset U$ (see Remark 3.1.3). Since $\left.T\right|_{V^{\prime \prime}}=0$, it is clear that $\left.\operatorname{trop}_{*}(T)\right|_{U^{\prime \prime}}=0$. Therefore

$$
\operatorname{trop}^{-1}\left(\operatorname{supp}\left(\operatorname{trop}_{*}(T)\right)\right) \subset \operatorname{supp}(T),
$$

concluding the proof of the proposition.

Example 7.1.7 In the case $U=N_{\mathbb{R}}$ and $V=\mathbb{T}^{\text {an }}$ our Correspondence Theorem 7.1.5 gives a new interpretation of the tropical currents introduced by Babaee and Huh [2, Chapter 3], [3, Section 2].

Let $\Delta$ be a polyhedron in $N_{\mathbb{R}}$ of dimension $p$ which is integral $\mathbb{R}$-affine, i.e. a polyhedron given by finitely many inequalities $\varphi \geq c$ with $\varphi \in \operatorname{Hom}_{\mathbb{Z}}(N, \mathbb{Z})$ and $c \in \mathbb{R}$. The argument map from polar coordinates induces an $\mathbb{S}$-equivariant fibration of the $\mathbb{S}$-invariant subset $\operatorname{trop}^{-1}(\Delta) \subset \mathbb{T}^{\text {an }}$ over a real torus of dimension $n-p$ with fibers of complex dimension $p$. Integration of a complex $(p, p)$-form over the fibers and then integrating the resulting function on the real torus with respect to the probability Haar measure defines a complex current $T_{\Delta} \in D^{n-p, n-p}\left(\mathbb{T}^{\mathrm{an}}\right)$. For details of the construction of $T_{\Delta}$, we refer to [3, Definition 2.3].

Let $\delta_{\Delta}$ denote the Lagerberg current of integration over $\Delta$ defined in $[11,3.6]$. The complex current $T_{\Delta}$ is by construction $\mathbb{S}$-invariant. We get furthermore trop ${ }_{*}\left(T_{\Delta}\right)=\delta_{\Delta}$ by a similar argument as in the proof of Lemma 4.2.5. Then Lemma 5.1.5(iii) yields that $T_{\Delta}$ is F-invariant.

If $C=(\mathscr{C}, m)$ is a weighted integral $\mathbb{R}$-affine polyhedral complex of pure dimension $p$ with weights $m$ in the sense of [11,3.1,3.3], then one defines

$$
T_{C}:=\sum_{\substack{\Delta \in \mathscr{C} \\ \operatorname{dim} \Delta=p}} m_{\Delta} T_{\Delta} \in D^{n-p, n-p}\left(\mathbb{T}^{\mathrm{an}}\right), \quad \delta_{C}:=\sum_{\substack{\Delta \in \mathscr{C} \\ \operatorname{dim} \Delta=p}} m_{\Delta} \delta_{\Delta} \in D^{n-p, n-p}\left(N_{\mathbb{R}}\right) .
$$

Since trop $*\left(T_{\Delta}\right)=\delta_{\Delta}$, we clearly have trop $*\left(T_{C}\right)=\delta_{C}$.

If $C$ is an effective tropical cycle, then $T_{C}$ is closed and positive by [3, Theorem 2.9]. The same is true for $\delta_{C}$ by $[11,3.7]$. We conclude that $T_{C}$ is the unique closed positive current in $D^{n-p, n-p}\left(\mathbb{T}^{\text {an }}\right)^{\mathbb{S}, F}$ such that $\operatorname{trop}_{*}\left(T_{C}\right)=\delta_{C}$.

\subsection{The analogue of the Skoda-El Mir Theorem for tropical toric varieties}

We will prove a tropical analogue of the Skoda-El Mir Theorem. In this subsection, $U$ is an open subset of $N_{\Sigma}$ and $E$ is the intersection of $U$ with a union of strata closures.

Let $T \in D^{p, p}(U \backslash E)$ be a positive Lagerberg current. We pick $\rho \in \Sigma$ and choose toric coordinates on $U_{\rho}$ as in Definition 6.1.2. By Proposition 5.2.6, the co-coefficients $T^{I J}$ are 
real Radon measures on $U_{\rho} \backslash\left(E^{I \cup J} \cup E\right)$ with total variation measure $\left|T^{I J}\right|$. We will consider the open immersion

$$
i_{E}^{I J}: U_{\rho} \backslash\left(E^{I \cup J} \cup E\right) \longrightarrow U_{\rho} \backslash E^{I \cup J} .
$$

Definition 7.2.1 Let $T \in D^{p, p}(U \backslash E)$ be a positive Lagerberg current. We say that $T$ is extendable by zero to $U$ if for any $\rho \in \Sigma$, any toric coordinates on $U_{\rho}$ and all subsets $I, J$ of $\{1, \ldots, n\}$ with $|I|=|J|=n-p$, the Radon measure $T^{I J}$ admits an image Radon measure with respect to $i_{E}^{I J}$ (see Appendix A). In other words, there is a (unique) Radon measure $i_{E}^{I J}\left(T^{I J}\right)$ on $U_{\rho} \backslash E^{I \cup J}$ which agrees with $T^{I J}$ on $U_{\rho} \backslash\left(E^{I \cup J} \cup E\right)$ such that $U_{\rho} \cap E \backslash E^{I \cup J}$ is a null set with respect to $i_{E}^{I J}\left(T^{I J}\right)$.

Lemma 7.2.2 If $T$ is extendable by zero to $U$, then there is a unique Lagerberg current $\widetilde{T} \in D^{p, p}(U)$ such that for any $\rho \in \Sigma$ and all toric coordinates on $U_{\rho}$, the co-coefficient $(\widetilde{T})^{I J}$ is induced by the Radon measure $i_{E}^{I J}\left(T^{I J}\right)$ for all $I, J$. Moreover, we have $\left.\widetilde{T}\right|_{U \backslash E}=T$ and the Lagerberg current $\widetilde{T}$ is positive.

In the above situation, we say that $\widetilde{T}$ is the extension of $T$ by zero to $U$.

Proof For $\rho \in \Sigma$ and a choice of toric coordinates, Remark 5.2.4 shows that there is a unique current $\widetilde{T} \in D^{p, p}\left(U_{\rho}\right)$ with co-coefficients induced by the Radon measures $i_{E}^{I J}\left(T^{I J}\right)$. Clearly, we have $\left.\widetilde{T}\right|_{U_{\rho} \backslash E}=\left.T\right|_{U_{\rho} \backslash E}$ and $\widetilde{T}$ does not depend on the choice of toric coordinates. Moreover, the extensions $\widetilde{T}$ constructed on the open covering $\left(U_{\rho}\right)_{\rho \in \Sigma}$ agree on overlapping. By glueing, we get a Lagerberg current on $U$ also denoted by $\widetilde{T}$. Using Lemma 6.1.4 for $A:=E \cap U_{\rho}$, we deduce easily that $\widetilde{T}$ is again a positive Lagerberg current.

Definition 7.2.3 Let $T \in D^{p, p}(U \backslash E)$ be a positive Lagerberg current. Generalizing Definition 6.2.1, we say that $T$ has $\mathbb{C}$-finite local mass on $U$ if for all $\rho \in \Sigma$ the co-coefficients $T^{I J}$, which may be seen as real Radon measures on $U_{\rho} \backslash\left(E \cup E^{I \cup J}\right)$, satisfy the condition that the Borel measures on $U_{\rho}$, given as the image measures

$$
j_{E}^{I J}\left(\left|T^{I, J}\right| \prod_{i \in I} e^{-u_{i}} \prod_{j \in J} e^{-u_{j}}\right)
$$

with respect to the open immersion $j_{E}^{I J}: U_{\rho} \backslash\left(E \cup E^{I \cup J}\right) \rightarrow U_{\rho}$, are locally finite on $U_{\rho}$.

Theorem 7.2.4 Let $T \in D^{p, p}(U \backslash E)$ be a closed positive Lagerberg current which has $\mathbb{C}$-finite local mass on $U$. Then $T$ is extendable by zero to $U$ and the extension $\widetilde{T}$ of $T$ by zero to $U$ from Lemma 7.2.2 is a closed positive Lagerberg current on $U$.

Proof We may assume that $U=U_{\rho}$ for some $\rho \in \Sigma$ and we choose toric coordinates as in Definition 6.1.2. Since the function $\prod_{i \in I} e^{-u_{i}} \prod_{j \in J} e^{-u_{j}}$ is locally finite on $U \backslash E^{I \cup J}$ and since $T$ has $\mathbb{C}$-finite local mass on $U$, the Borel measure $i_{E}^{I J}\left(\left|T^{I J}\right|\right)$ is locally finite on $U \backslash E^{I \cup J}$ and hence $T$ is extendable by zero to $U$. By Lemma 7.2.2, the extension $\widetilde{T}$ of $T$ by zero to $U$ is a positive Lagerberg current on $U$.

Let $V:=\operatorname{trop}^{-1}(U)$ and let $D:=\operatorname{trop}^{-1}(E)$. Note that $D$ is the intersection of the open subset $V$ of $X_{\Sigma}^{\text {an }}$ with a union of strata closures and hence is a closed analytic subset of the complex toric manifold $X_{\Sigma}^{\text {an }}$. By our correspondence theorem (Theorem 7.1.5), there is a unique closed positive current $S \in D^{p, p}(V \backslash D)_{\mathrm{cl},+}^{\mathbb{S}, F}$ with $\operatorname{trop}_{*}(S)=T$. Using that $T$ has $\mathbb{C}$-finite local mass on $U$, it follows from (6.4) that $S$ has finite local mass on $V$. The complex Skoda-El Mir Theorem (see [9, Theorem III.2.3]) shows that the extension $\widetilde{S}$ of $S$ by zero to $V$ is a closed positive current on $V$. By construction, we have $\operatorname{trop}_{*}(\widetilde{S})=\widetilde{T}$ and hence $\widetilde{T}$ is a closed Lagerberg current. 
Corollary 7.2.5 Let $T \in D^{p, p}(U)$ be positive and closed with canonical decomposition $T=\sum_{\sigma \in \Sigma} T_{\sigma}$ from Theorem 6.1.6. Then every Lagerberg current $T_{\sigma}$ is positive and closed.

Proof By Theorem 6.1.6, every $T_{\sigma}$ is a positive Lagerberg current. We know from Proposition 7.1.2 that the closed positive Lagerberg current $T$ has $\mathbb{C}$-finite local mass. These two facts show that every $T_{\sigma}$ has $\mathbb{C}$-finite local mass.

We prove that $T_{\sigma}$ is closed by induction on the dimension of $\sigma$. By construction $T_{\{0\}}$ is the extension by zero of $\left.T\right|_{N_{\mathbb{R}} \cap U}$. Hence $T_{\{0\}}$ is closed by Theorem 7.2.4. We assume now that $T_{\tau}$ is closed for all $\tau$ with $\operatorname{dim}(\tau)<\operatorname{dim}(\sigma)$. Hence

$$
T_{\sigma}^{\prime}:=T-\sum_{\operatorname{dim}(\tau)<\operatorname{dim}(\sigma)} T_{\tau}
$$

is closed. Since $\left.T_{\sigma}\right|_{U_{\sigma}}=\left.T_{\sigma}^{\prime}\right|_{U_{\sigma}}$ we deduce that $\left.T_{\sigma}\right|_{U_{\sigma}}$ is closed. As $T_{\sigma}$ is the extension by zero of this last current, Theorem 7.2.4 implies that $T_{\sigma}$ is closed.

Acknowledgements We are deeply grateful to Florent Martin for his numerous inputs at the beginning of this project. We are grateful to Roberto Gualdi for his comments on a previous version and for pointing out the reference [3]. We thank the referee for helpful comments.

Funding Open Access funding enabled and organized by Projekt DEAL.

Open Access This article is licensed under a Creative Commons Attribution 4.0 International License, which permits use, sharing, adaptation, distribution and reproduction in any medium or format, as long as you give appropriate credit to the original author(s) and the source, provide a link to the Creative Commons licence, and indicate if changes were made. The images or other third party material in this article are included in the article's Creative Commons licence, unless indicated otherwise in a credit line to the material. If material is not included in the article's Creative Commons licence and your intended use is not permitted by statutory regulation or exceeds the permitted use, you will need to obtain permission directly from the copyright holder. To view a copy of this licence, visit http://creativecommons.org/licenses/by/4.0/.

\section{Appendix A: Reminder about Radon and regular Borel measures}

For the convenience of the reader, we gather the used conventions about Radon measures and some basic facts. In this paper, we deal only with measures on locally compact Hausdorff spaces which have a countable basis, so let us consider such a space $Y$.

For $\mathbb{K} \in\{\mathbb{R}, \mathbb{C}\}$ and a compact subset $Z$ of $Y$, we write $C_{Z}^{0}(Y, \mathbb{K})$ for the space of $\mathbb{K}$ valued continuous functions on $Y$ with support in $Z$ equipped with the topology induced by the supremum norm. The space $C_{c}^{0}(Y, \mathbb{K})$ of $\mathbb{K}$-valued continuous functions on $Y$ with compact support is the direct limit of the spaces $C_{Z}^{0}(Y, \mathbb{K})$. We equip $C_{c}^{0}(Y, \mathbb{K})$ with the direct limit topology in the category of locally convex topological vector spaces (see [4, III $\S 1$ no 1$])$.

We define the space of real Radon measures on $Y$ as the topological dual of $C_{c}^{0}(Y, \mathbb{R})$. Observe that our Radon measures are precisely the measures considered by Bourbaki [4, III $\S 1$ no $3+5]$. If $\mu: C_{c}^{0}(Y, \mathbb{R}) \rightarrow \mathbb{R}$ is linear with $\mu(f) \geq 0$ for all $0 \leq f \in C_{c}^{0}(Y, \mathbb{R})$, then we call $\mu$ a positive linear functional. Note that $\mu$ is then continuous and gives rise to a positive Radon measure. The Riesz representation theorem shows that there is a unique regular Borel measure $\mu^{B}$ on $Y$ such that

$$
\mu(f)=\int_{Y} f d \mu^{B}
$$


for all $f \in C_{c}^{0}(Y, \mathbb{R})$. Recall that a Borel measure $\lambda$ on $Y$ is regular if and only if it is locally finite, i.e. any point has an open neighborhood $U$ with $\lambda(U)<\infty$ [8, Prop. 7.2.3 and 7.2.5]. In contrast to Radon measures, we always assume that a Borel measure is positive.

A real Radon measure $\mu: C_{c}^{0}(Y, \mathbb{R}) \rightarrow \mathbb{R}$ can be written as the difference $\mu=\mu_{+}-\mu_{-}$ of positive Radon measures $\mu_{ \pm}$which are induced by locally finite (positive) Borel measures $\mu_{ \pm}^{B}$. The Hahn-Jordan decomposition theorem tells us that the Borel measures $\mu_{ \pm}^{B}$ are unique if one requires them to be minimal. The positive Radon measure $|\mu|:=\mu_{+}+\mu_{-}$is called the total variation measure of $\mu$. We call $|\mu|^{B}(Y)=\mu_{+}^{B}(Y)+\mu_{-}^{B}(Y)$ the total variation of $\mu$. Observe that $\mu_{+}^{B}-\mu_{-}^{B}$ is not necessarily a signed Borel measure as it has only well defined finite values on $\mu_{ \pm}^{B}$-finite Borel sets. The standard example is the real Radon measure on $\mathbb{R}$ with density function $\sin (x)$ with respect to the Lebesgue measure which is not a signed Borel measure as the total space $\mathbb{R}$ has no well-defined mass.

A complex Radon measure on $Y$ is defined as a continuous linear functional $\mu: C_{c}^{0}(Y, \mathbb{C}) \rightarrow$ $\mathbb{C}$. It is clear that the real and imaginary part of $\mu$ are real Radon measures. From this, we conclude as above that $\mu$ corresponds locally on an open subset $U$ of $Y$ to a complex Borel measure $\mu_{U}^{B}$. The total variation measure of $\mu_{U}^{B}$ is the unique Borel measure $\left|\mu_{U}^{B}\right|$ on $U$ such that there is a Borel measurable function $\theta: U \rightarrow \mathbb{R}$ with $d \mu_{U}^{B}=e^{i \theta} d\left|\mu_{U}^{B}\right|[21,6.1$, 6.12]. The Borel measures $\left|\mu_{U}^{B}\right|$ glue to a Borel measure on $Y$ which is associated to a Radon measure $|\mu|$ on $Y$ called the total variation measure of the Radon measure $\mu$. We call $|\mu|^{B}(Y)$ the total variation of $\mu$.

Let $g: X \rightarrow Y$ be a continuous map of locally compact Hausdorff spaces which admit a countable basis. We say that a real or complex Radon measure $\mu$ on $X$ admits an image Radon measure under $g$ if the image measure $g\left(|\mu|^{B}\right)$ is a locally finite Borel measure.

Assume that the real or complex Radon measure $\mu$ on $X$ admits an image Radon measure under $g$. If $\mu$ is a real Radon measure on $X$, then we write $\mu=\mu_{+}-\mu_{-}$as above. By our assumption the image measures $f\left(\mu_{+}^{B}\right)$ and $f\left(\mu_{-}^{B}\right)$ are locally finite and define Radon measures $f(\mu)_{+}$and $f(\mu)_{-}$on $Y$. We call the real Radon measure $f(\mu):=f(\mu)_{+}-f(\mu)_{-}$ the image measure of $\mu$ under $f$. If $\mu$ is a complex Radon measure $\mu$ on $X$, we define the image measure $f(\mu)$ by treating the real and the imaginary part of $\mu$ separately.

It is straightforward to check that real and complex Radon measures always admit image Radon measures under proper maps.

\section{References}

1. Ash, A., Mumford, D., Rapoport, M., Tai, Y.-S.: Smooth compactifications of locally symmetric varieties. Cambridge Mathematical Library, 2nd edn. Cambridge University Press, Cambridge (2010). With the collaboration of Peter Scholze

2. Babaee, F.: Complex Tropical Currents. PhD thesis, Université Bordeaux and Università degli Studi di Padova (2014). www.tel.archives-ouvertes.fr/tel-01136662

3. Babaee, Farhad: Huh, June: A tropical approach to a generalized Hodge conjecture for positive currents. Duke Math. J. 166(14), 2749-2813 (2017)

4. Bourbaki, N.: Éléments de mathématique. Intégration. Chapitres 1-4. Actualités Scientifiques et Industrielles, No. 1175. Hermann, Paris (1965)

5. Bourbaki, N.: Éléments de mathématique. Topologie générale. Chapitres 1-4. Hermann, Paris (1971)

6. Burgos G., José I., Philippon, P., Sombra, M.: Arithmetic geometry of toric varieties. Metrics, measures and heights. Astérisque, 360:vi+222 (2014)

7. Chambert-Loir, A., Ducros, A.: Formes différentielles réelles et courants sur les espaces de Berkovich (2012). arXiv:1204.6277

8. Cohn, Donald L.: Measure theory. Birkhäuser Boston Inc, Boston, MA (1993)

9. Demailly, J.-P.: Complex analytic and differential geometry, eBook, Version June 21st 2012. www-fourier. ujf-grenoble.fr/ demailly/manuscripts/agbook.pdf 
10. Dieudonné, Jean: Schwartz, Laurent: La dualité dans les espaces $(\mathcal{F})$ et $(\mathcal{L} \mathcal{F})$. Ann. Inst. Fourier $($ Grenoble) 1(61-101), 1949 (1950)

11. Gubler, W.: Forms and currents on the analytification of an algebraic variety (after Chambert-Loir and Ducros). In: Baker, M., Payne, S. (eds) Non-archimedean and Tropical Geometry, Simons Symposia, pp. 1-30, Springer, Switzerland (2016)

12. Gubler, Walter: Künnemann, Klaus: A tropical approach to nonarchimedean Arakelov geometry. Algebra Number Theory 11(1), 77-180 (2017)

13. Jell, P.: Tropical cohomology with integral coefficients for analytic spaces (2019). arXiv:1909.12603

14. Jell, Philipp: Shaw, Kristin, Smacka, Jascha: Superforms, tropical cohomology, and Poincaré duality. Adv. Geom. 19(1), 101-130 (2019)

15. Kajiwara, T.: Tropical toric geometry. In: Toric topology, volume 460 of Contemp. Math., pp. $197-207$. Amer. Math. Soc., Providence, RI (2008)

16. Lagerberg, Aron: Super currents and tropical geometry. Math. Z. 270(3-4), 1011-1050 (2012)

17. Oda, T.: Convex bodies and algebraic geometry. Ergebnisse der Mathematik und ihrer Grenzgebiete (3), vol. 15. Springer, Berlin (1988)

18. Payne, Sam: Analytification is the limit of all tropicalizations. Math. Res. Lett. 16(3), 543-556 (2009)

19. Tyrrell Rockafellar, R.: Convex Analysis. Princeton Mathematical Series, No. 28. Princeton University Press, Princeton, N.J. (1970)

20. Rudin, Walter: Principles of mathematical analysis, 2nd edn. McGraw-Hill Book Co., New York (1964)

21. Rudin, Walter: Real and complex analysis. McGraw-Hill Book Co., New York(1966)

22. Rudin, Walter: Functional Analysis. McGraw-Hill Book Co., New York (1973)

23. Warner, Frank W.: Foundations of differentiable manifolds and Lie groups. vol. 94. Springer-Verlag, New York-Berlin (1983)

Publisher's Note Springer Nature remains neutral with regard to jurisdictional claims in published maps and institutional affiliations. 